\title{
Peridynamics for geometrically nonlinear analysis of three-dimensional beam structures
}

Cong Tien Nguyen and Selda Oterkus*

Department of Naval Architecture, Ocean and Marine Engineering, University of Strathclyde, Glasgow, G11XQ, UK

*Corresponding author: selda.oterkus@ strath.ac.uk

\begin{abstract}
This study presents a novel peridynamic model for geometrically nonlinear analysis of threedimensional beam structures. The formulations of nonlinear strain energy densities for a beam is obtained by using Total Lagrange formulation. The peridynamic formulations for beam structures are obtained based on the principle of virtual displacements. The capability of the proposed peridynamic model is demonstrated by considering large deformations of straight beams and curved beams subjected to different loading conditions. To further demonstrate the capabilities of the proposed nonlinear model, damages on a dry spaghetti subjected to different loading conditions are simulated.
\end{abstract}

Keywords: Peridynamics, Nonlinear, Beam, Damage, Total Lagrange formulations 


\section{Nomenclature}

\section{Latin Letters}

${ }^{0} A$

${ }_{0}^{t} a_{1},{ }_{0}^{t} a_{2}, \ldots,{ }_{0}^{t} a_{15}$

${ }_{0}^{t} a_{1}^{P D},{ }_{0}^{t} a_{2}^{P D}, \ldots,{ }_{0}^{t} a_{15}^{P D}$

${ }_{0} \underline{a}_{1},{ }_{0} \underline{a}_{2}, \ldots,{ }_{0} \underline{a}_{15}$

${ }_{0} \underline{a}_{1}^{P D},{ }_{0} \underline{a}_{2}^{P D}, \ldots,{ }_{0} \underline{a}_{15}^{P D}$

${ }^{t} \mathbf{b}_{(k)}^{L},{ }^{t} \mathbf{b}_{(k)}^{G}$

$C_{a x}$

$C_{b y}, C_{b z}$

$C_{\text {sh }}$

$C_{t}$

E

${ }_{0} \underline{\boldsymbol{e}}_{x x},{ }_{0} \underline{\boldsymbol{e}}_{y y},{ }_{0} \underline{\boldsymbol{e}}_{z z},{ }_{0} \underline{\boldsymbol{e}}_{x y}$,

${ }_{0} \underline{\boldsymbol{e}}_{y z},{ }_{0} \underline{\boldsymbol{e}}_{x z}$

${ }_{0}^{t} \mathbf{f}_{(k)(j)}^{L}$ or $\left({ }_{0}^{t} f_{(k)(j)}^{u},{ }_{0}^{t} f_{(k)(j)}^{v}\right.$,

${ }_{0}^{t} f_{(k)(j)}^{w},{ }_{0}^{t} f_{(k)(j)}^{\theta_{x}},{ }_{0}^{t} f_{(k)(j)}^{\theta_{y}}$,

$\left.{ }_{0}^{t} f_{(k)(j)}^{\theta_{z}}\right)$

${ }_{0}^{t} \mathbf{f}_{(k)(j)}^{G}$ or $\left({ }_{0}^{t} f_{(k)(j)}^{u_{1}},{ }_{0}^{t} f_{(k)(j)}^{u_{2}}\right.$,

${ }_{0}^{t} f_{(k)(j)}^{u_{3}},{ }_{0}^{t} f_{(k)(j)}^{\theta_{1}},{ }_{0}^{t} f_{(k)(j)}^{\theta_{2}}$,

$\left.{ }_{0}^{t} f_{(k)(j)}^{\theta_{3}}\right)$

$G$

$G_{c}$

$g_{c}$

$\bar{g}_{(k)(j)}$

${ }^{0} \mathbf{H}_{(k)}$
$I_{y y}, I_{z z}$
$k_{s}$

The cross-section area of a beam in the initial configuration

The terms that are used to calculate nonlinear SED in CCM given in Eq. (22). These terms are calculated by using displacements and rotations at time $t$.

The terms that are used to calculate nonlinear SED in PD given in Eq. (29-28). These terms are calculated by using displacements and rotations at time $t$.

The incremental terms that are used to calculate nonlinear SED in CCM given in Eq. (22). These incremental terms are calculated by using incremental displacements and rotations.

The incremental terms are given in Eq. (29-28) that are used to calculate nonlinear SED in PD. These incremental terms are calculated by using incremental displacements and rotations.

Applied body forces in the local, global coordinate system, respectively.

PD bond constant for axial deformations.

PD bond constants for bending deformations.

PD bond constant for shear deformations.

PD bond constant for torsional deformations.

Elastic modulus.

Incremental linear strain from time $t$ to time $t+\Delta t$, with respect to the initial configuration.

Vectors of PD force densities at time $t$ in the local coordinate system.

Vectors of PD force densities at time $t$ in the global coordinate system.

Shear modulus of material.

The critical energy release rate of material.

The average critical energy release rate for one interaction.

Energy release rate for interaction between material points $k$ and $j$ in peridynamics.

Coordinate transformation matrix.

Moments of inertia.

Shear correction factor. 


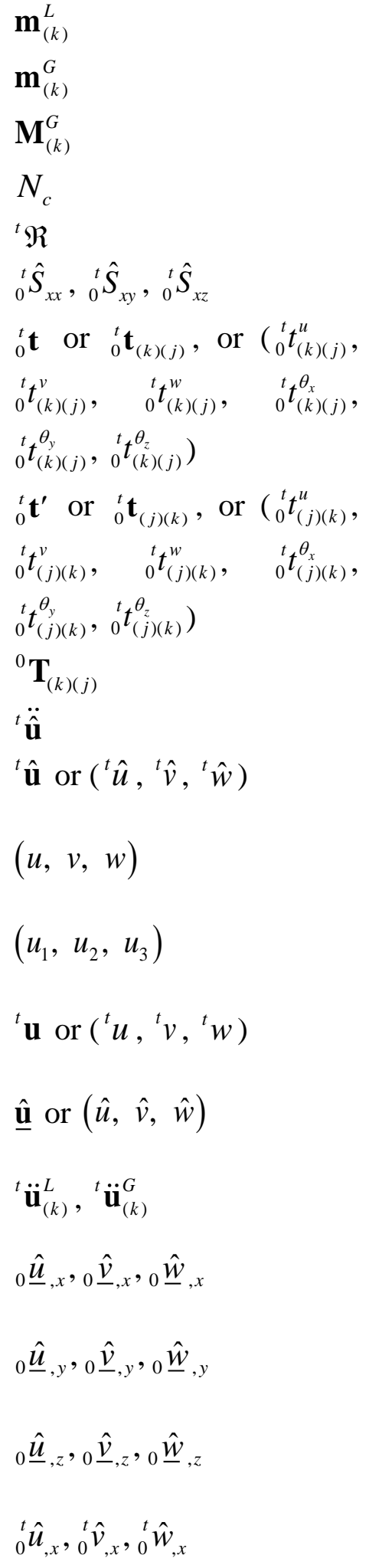

Mass matrix of material point $k$ in local coordinates.

Mass matrix of material point $k$ in global coordinates.

Mass stable vector of material point $k$ in global coordinates.

Total number of interactions passing through a unit crack surface External virtual work at time $t$.

Components of Second Piola-Kirchhoff (SPK) stress at time $t$.

PD force density that material point $j$ exerts on material point $k$

PD force density that material point $k$ exerts on material point $j$

The transformation matrix for 6 degrees of freedom.

Vector of acceleration at time $t$.

Displacements at time $t$ of a material point located at $\left({ }^{0} x,{ }^{0} y,{ }^{0} z\right)$ in the local coordinate system.

Displacements of a material point located at $\left({ }^{0} x, 0,0\right)$ in the local coordinates.

Displacements of a material point located at $\left({ }^{0} x, 0,0\right)$ in the global coordinates.

Displacements of a material point located at $\left({ }^{0} x, 0,0\right)$ in the local coordinate system.

Incremental displacements of a material point located at $\left({ }^{0} x,{ }^{0} y,{ }^{0} z\right)$ in the local coordinate system.

Acceleration vectors of material point $k$ in local, global coordinate systems, respectively.

Derivatives of the incremental displacement $\underline{\hat{u}}, \underline{\hat{v}}, \underline{\hat{w}}$ with respect to the initial coordinate ${ }^{0} x$.

Derivatives of the incremental displacement $\underline{\hat{u}}, \underline{\hat{v}}, \underline{\hat{w}}$ with respect to the initial coordinate ${ }^{0} y$.

Derivatives of the incremental displacement $\underline{\hat{u}}, \underline{\hat{v}}, \underline{\hat{w}}$ with respect to the initial coordinate ${ }^{0} z$.

Derivatives of the displacement ${ }^{t} \hat{u},{ }^{t} \hat{v},{ }^{t} \hat{w}$ at time $t$ with respect to the initial coordinate ${ }^{0} x$. 


$$
\begin{aligned}
& { }_{0}^{t} \hat{u}_{, y},{ }_{0}^{t} \hat{v}_{, y},{ }_{0}^{t} \hat{w}_{, y} \\
& { }_{0} \hat{u}_{, z},{ }_{0}{ }_{0} \hat{v}_{, z},{ }_{0}^{t} \hat{w}_{, z} \\
& { }^{0} V \\
& { }^{0} V_{(j)} \\
& \left({ }^{0} \mathbf{V}_{r},{ }^{0} \mathbf{V}_{s},{ }^{0} \mathbf{V}_{t}\right) \\
& \left({ }^{t} \mathbf{V}_{r},{ }^{t} \mathbf{V}_{s},{ }^{t} \mathbf{V}_{t}\right) \\
& \left({ }^{0} \mathbf{V}_{r}^{G},{ }^{0} \mathbf{V}_{s}^{G},{ }^{0} \mathbf{V}_{t}^{G}\right) \\
& \left({ }^{t} \mathbf{V}_{r}^{G},{ }^{t} \mathbf{V}_{s}^{G},{ }^{t} \mathbf{V}_{t}^{G}\right) \\
& \mathbf{V}_{s}, \mathbf{V}_{t} \\
& W^{N L} \\
& \bar{W}^{N L} \\
& \bar{W}_{\text {axial }}^{N L}, \bar{W}_{\text {bending }}^{N L}, \bar{W}_{\text {shear }}^{N L} \text { and } \\
& \bar{W}_{\text {torsion }}^{N L} \\
& \bar{W}^{N L P D} \\
& \bar{W}_{\text {axial }}^{N L P D}, \bar{W}_{\text {bending }}^{N L P D}, \bar{W}_{\text {shear }}^{N L P D} \text { and } \\
& \bar{W}_{\text {torsion }}^{N L P D} \\
& { }^{0} \mathbf{x}^{L} \text { or }\left({ }^{0} x,{ }^{0} y,{ }^{0} z\right) \\
& { }^{t} \mathbf{x}^{L} \text { or }\left({ }^{t} x,{ }^{t} y,{ }^{t} z\right) \\
& { }^{0} \mathbf{x}^{G} \text { or }\left({ }^{0} x_{1},{ }^{0} x_{2},{ }^{0} x_{3}\right) \\
& { }^{t} \mathbf{x}^{G} \text { or }\left({ }^{t} x_{1},{ }^{t} x_{2},{ }^{t} x_{3}\right) \\
&
\end{aligned}
$$

\section{Greek Letters}

$\delta$

$$
{ }_{0}^{t} \hat{\varepsilon}_{x x},{ }_{0}^{t} \hat{\varepsilon}_{x y},{ }_{0} \hat{\varepsilon}_{x z}
$$$$
\theta_{x}, \quad \theta_{y}, \quad \theta_{z}
$$$$
\theta_{1}, \quad \theta_{2}, \quad \theta_{3}
$$$$
\underline{\boldsymbol{\theta}}={ }^{t+\Delta t} \boldsymbol{\theta}-{ }^{t} \boldsymbol{\theta}
$$$$
=\left[\begin{array}{lll}
\underline{\theta}_{x} & \underline{\theta}_{y} & \underline{\theta}_{z}
\end{array}\right]^{T}
$$

$\psi_{(k)(j)}$
Derivatives of the displacement ${ }^{t} \hat{u},{ }^{t} \hat{v},{ }^{t} \hat{w}$ at time $t$ with respect to the initial coordinate ${ }^{0} y$.

Derivatives of the displacement ${ }^{t} \hat{u},{ }^{t} \hat{v},{ }^{t} \hat{w}$ at time $t$ with respect to the initial coordinate ${ }^{0} z$.

Volume in the original configuration.

The volume of material point $j$ in the original configuration.

Director vectors in the initial configuration. These vectors are measured in the beam's local coordinates.

Director vectors at time $t$. These vectors are measured in the beam's local coordinates.

Director vectors in the initial configuration. These vectors are measured in the global coordinates.

Director vectors at time $t$. These vectors are measured in the global coordinates.

Incremental director vectors (with respect to the beam's local coordinates) from time $t$ to time $t+\Delta t$.

Nonlinear strain energy density in classical continuum mechanics.

Nonlinear strain energy density of a beam in classical continuum mechanics.

Nonlinear strain energy densities for axial, bending, shear and torsional deformations of a beam in classical continuum mechanics.

Nonlinear strain energy density for a beam in peridynamics.

Nonlinear strain energy densities for axial, bending, shear and torsional deformations of a beam in peridynamics.

Local coordinates in the initial (undeformed) configuration.

Local coordinates in the deformed configuration at time $t$.

Global coordinates in the initial (undeformed) configuration.

Global coordinates in the deformed configuration at time $t$.

\section{Horizon size}

Components of Green Lagrange strain at time $t$. These strains are measured with respect to the initial configuration.

Rotational degrees of freedom in the local coordinates.

Rotational degrees of freedom in the global coordinates.

Incremental rotations in local coordinates.

Damage parameter to represent the interaction state of a bond $\left(\psi_{(k)(j)}=0\right.$ or 1$)$. 


$$
\begin{aligned}
& v \\
& \varphi_{(k)} \\
& { }_{0}^{t} \Phi_{(k)(j)} \text { and }{ }_{0}^{t} \Phi_{(j)(k)} \\
& { }^{0} \xi_{(j)(k)} \\
& \rho
\end{aligned}
$$

\section{Acronyms}

ADR

$\mathrm{CCM}$

$\operatorname{DOF}(s)$

FEA

GL

L-FEA

NL-FEA

NL-PD

$\mathrm{PD}$

SED

SPK

$3 \mathrm{D}$
Poisson's ratio

Local damage parameter (or damage index) represents the level of damage on the structure $\left(0 \leq \varphi_{(k)} \leq 1\right)$.

Micropotentials at time $t$ of the interaction between material points $k$ and $j$.

The bond length between material points $k$ and $j$ in the initial configuration.

Mass density

Adaptive Dynamic Relaxation

Classical Continuum Mechanics

Degree(s) of freedom

Finite Element Analysis

Green Lagrange strain

Linear Finite Element Analysis

Nonlinear Finite Element Analysis

Nonlinear Peridynamics

Peridynamics

Strain Energy Density

Second Piola-Kirchhoff stress

Three dimensions 


\section{Introduction}

Computational modeling of engineering structures in a three-dimensional (3D) fashion can be computationally expensive especially for complex structures. Therefore, structural simplification can be used to reduce the computational cost. When a structure has a dimension that is much smaller than the other dimensions, the formulations for shell structures can be used [1-8]. When a structure has two dimensions that are much smaller than the other, the formulations for beam structures can be used [7, 9].

When structures are slender, they can experience large deformations even with small loads. Therefore, it is quite often to observe large displacements, large rotations, but small elastic strains behaviours of beam structure in practice. In these cases, the geometrically nonlinear analysis can be used.

In nonlinear finite element analysis (FEA) within the Classical Continuum Mechanics (CCM), the equation of motion is obtained from the linearization of the principle of virtual displacements [7]. The solution in the nonlinear analysis is obtained after several iterations when some convergent criteria are satisfied [10]. However, because of using partial-differential equations that become invalid in the presence of discontinuities, the classical continuum mechanics face mathematical and conceptual difficulties in terms of predicting crack nucleation and growth, especially for multiple crack paths.

Peridynamics (PD) represents the material behaviour by using integro-differential equations, which are valid in both continuous and discontinuous models [11-14]. Therefore, discontinuities such as crack propagation and branching can be naturally involved in the PD analysis without any special treatment $[12,15-20]$.

To date, PD theory has been implemented in many fields such as nonlinear elastic or inelastic material responses [21-26], multiphysics [27-38], multiscale modelling [39, 40], orthotropic material responses [41-44], and fatigue cracking [45-47]. Moreover, it can also be either combined with finite element analysis (FEA) [41, 48-50] or implemented in the FEA framework [31, 51-54].

In terms of structural simplifications, the first simplified two-dimensional (2D) PD model was introduced by Silling and Bobaru [55], and later by Madenci and Oterkus [15]. Later, O'Grady and Foster [56], Diyaroglu et al. [57] introduced PD models for capturing bending and transverse shear deformations of plates. Later, Nguyen and Oterkus [35] developed an ordinary state-based PD model for three-dimensional (3D) shell structures with six degrees of freedom (DOF). The PD shell model can also be used to predict damages on ship structures [58]. Recently, Yang, et al. [59], [60, 61] also developed state-based PD models for the Kirchhoff plate formulation and functionally graded materials.

To investigate slender structures, Silling et al. [62] introduced a PD model to capture axial deformations for 1D bars. Later, O'Grady and Foster [63], Diyaroglu et al. [57, 64] introduced PD models to capture bending and transverse shear deformations for beams. Yang, et al. [65] developed a state-based peridynamic formulation for functionally graded Euler-Bernoulli beams. Recently, Nguyen and Oterkus [66] developed a PD model for 3D beam structures with six DOFs. The PD beam model can be used to predict the damages in complex beam structures such as jacket 
platforms. However, the PD model developed by the authors is based on a small deformation assumption which can be applied for linear elastic material responses. Therefore, in this study, a novel PD model for geometrically nonlinear analysis of beam structures with 6 DOFs is proposed. The nonlinear PD formulations and equations of motion are obtained based on the principle of virtual displacements by using the Total Lagrange formulation. The numerical procedure in PD nonlinear analysis for beams is provided. The capabilities of the nonlinear PD model are verified by considering various examples of straight beams and curved beams subjected to large deformations. The developed nonlinear PD model is used to predict damages on a straight beam, which is a representation of dry spaghetti, subjected to different loading conditions.

This paper is organized as follows. Section 2 presents the principle of virtual displacement in classical continuum mechanics (CCM). Section 3 presents nonlinear beam kinematics in classical continuum mechanics. Section 4 presents nonlinear beam kinematics in peridynamics. The criterion for damage prediction in PD for beam structure is presented in Section 5, followed by the numerical procedure is present in Section 6. The numerical results are presented in Section 7 and the conclusion is given in Section 8.

\section{Total Lagrange formulations}

In this section, the Total Lagrange (TL) formulations in classical continuum mechanics are presented. As shown in Fig. 1.Error! Reference source not found. for the structure configurations in the Cartesian coordinate system at different times, $P$ is a material point on the structure, and its coordinates at time $t=0$ are denoted by $\left({ }^{0} x,{ }^{0} y,{ }^{0} z\right)$. The coordinates of this point at time $t$ and $t+\Delta t$ are denoted by $\left({ }^{t} x,{ }^{t} y,{ }^{t} z\right)$ and $\left({ }^{t+\Delta t} x,{ }^{t+\Delta t} y,{ }^{t+\Delta t} z\right)$, respectively. The coordinates of $P$ at time $t$ and $t+\Delta t$ can be represented as

${ }^{t} \mathbf{x}={ }^{0} \mathbf{x}+{ }^{t} \hat{\mathbf{u}}$

${ }^{t+\Delta t} \mathbf{x}={ }^{0} \mathbf{X}+{ }^{t+\Delta t} \hat{\mathbf{u}}$

with

${ }^{0} \mathbf{x}=\left[\begin{array}{c}0 \\ 0 \\ { }^{0} y \\ { }^{0} z\end{array}\right] ;{ }^{t} \mathbf{x}=\left[\begin{array}{c}{ }^{t} x \\ { }^{t} y \\ { }^{t} z\end{array}\right] ;{ }^{t+\Delta t} \mathbf{x}=\left[\begin{array}{c}t+\Delta t \\ { }^{t+\Delta t} y \\ { }^{t+\Delta t} z\end{array}\right] ;{ }^{t} \hat{\mathbf{u}}=\left[\begin{array}{c}{ }^{t} \hat{u} \\ { }^{t} \hat{v} \\ { }^{t} \hat{w}\end{array}\right] ;{ }^{t+\Delta t} \hat{\mathbf{u}}=\left[\begin{array}{c}t+\Delta t \\ { }^{t+\Delta t} \hat{v} \\ { }^{t+\Delta t} \hat{w}\end{array}\right]$

where ${ }^{t} \hat{\mathbf{u}}$ and ${ }^{t+\Delta t} \hat{\mathbf{u}}$ represent the displacement vectors of material point $P$ at time $t$ and $t+\Delta t$, respectively. The relationship between vectors ${ }^{t} \hat{\mathbf{u}}$ and ${ }^{t+\Delta t} \hat{\mathbf{u}}$ can be represented as

${ }^{t+\Delta t} \hat{\mathbf{u}}={ }^{t} \hat{\mathbf{u}}+\underline{\hat{\mathbf{u}}}$

with

$\underline{\hat{\mathbf{u}}}=\left[\begin{array}{lll}\underline{\hat{u}} & \underline{\hat{v}} & \underline{\hat{w}}\end{array}\right]$

where $\underline{\hat{\mathbf{u}}}$ represents the vector of the incremental displacements from time $t$ to $t+\Delta t$ of a material point $P$. 


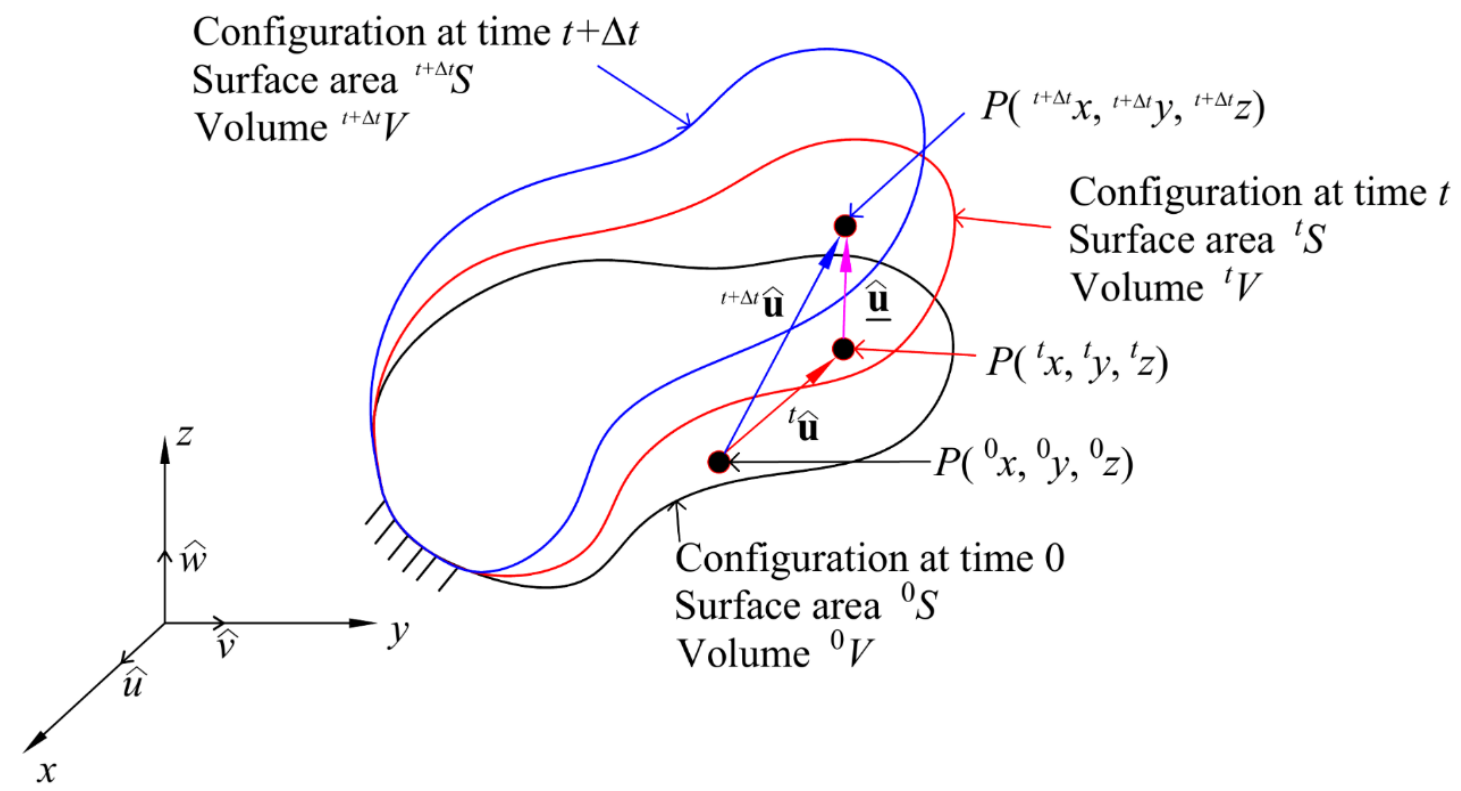

Fig. 1. Configurations of the structure in the Cartesian coordinates at time $t$ and $t=0$

According to Bathe [7], the equation of motion in the Total Lagrangian Formulation for a beam using the explicit time integration scheme can be written as

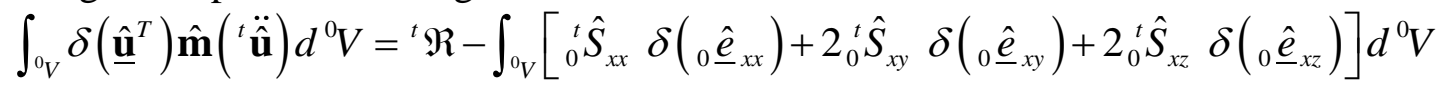

where $\hat{\mathbf{m}}$ represents the mass matrix, ${ }^{t} \ddot{\hat{\mathbf{u}}}$ represents the acceleration vector at time $t$ of the material point located at $\left({ }^{0} x,{ }^{0} y,{ }^{0} z\right)$, $\underline{\hat{\mathbf{u}}}$ represents the incremental displacement vector of the material point located at $\left({ }^{0} x,{ }^{0} y,{ }^{0} z\right)$ as given in Eq. $(2 \mathrm{~b}), \underline{\hat{\mathbf{u}}}^{T}$ represents the transpose of vector $\underline{\hat{\mathbf{u}}}$. The first term on the left-hand side of Eq. (3) represents the virtual work of the inertia forces [7]. The term, $\delta(\cdot)$ , represents the virtual value. The terms, ${ }_{0} \underline{\hat{e}}_{x x},{ }_{0} \underline{\hat{e}}_{x y},{ }_{0} \underline{\hat{e}}_{x z}$, represent the nonzero linear components of the incremental strains for a beam structure which can be written as [7]

${ }_{0} \underline{\hat{e}}_{x x}={ }_{0} \underline{\hat{u}}_{, x}+{ }_{0}^{t} \hat{u}_{, x 0} \underline{\hat{u}}_{, x}+{ }_{0} \hat{v}_{, x 0} \hat{\underline{\hat{v}}}_{, x}+{ }_{0}^{t} \hat{w}_{, x 0} \underline{\hat{w}}, x_{, x}$

${ }_{0} \underline{\hat{\hat{e}}}_{x y}=\frac{1}{2}\left({ }_{0} \hat{\hat{u}}_{, y}+{ }_{0} \hat{\underline{\hat{v}}}, x\right)+\frac{1}{2}\left(\begin{array}{l}{ }_{0}^{t} \hat{u}_{, x 0} \underline{\hat{u}}_{, y}+{ }_{0}^{t} \hat{u}_{, y 0} \hat{\hat{u}}_{, x}+{ }_{0}^{t} \hat{v}_{, x 0} \hat{\hat{v}}_{, y}+{ }_{0}^{t} \hat{v}_{, y 0} \hat{\hat{v}}_{, x} \\ +{ }_{0}^{t} \hat{w}_{, x} 0 \underline{\hat{w}}_{, y}+{ }_{0}^{t} \hat{w}_{, y 0} \hat{\underline{\hat{w}}}_{, x}\end{array}\right)$

${ }_{0} \underline{\hat{e}}_{x z}=\frac{1}{2}\left({ }_{0} \underline{\hat{u}}_{, z}+{ }_{0} \underline{\hat{w}}_{, x}\right)+\frac{1}{2}\left(\begin{array}{l}{ }_{0}^{t} \hat{u}_{, x 0} \underline{\hat{u}}_{, z}+{ }_{0}^{t} \hat{u}_{, z 0} \underline{\hat{u}}_{, z}+{ }_{0}^{t} \hat{v}_{, x 0} \underline{\hat{v}}_{, z}+{ }_{0}^{t} \hat{v}_{, z 0} \underline{\hat{v}}_{, z} \\ +{ }_{0}^{t} \hat{w}_{, x 0} \underline{\underline{\hat{w}}}_{, z}+{ }_{0}^{t} \hat{w}_{, z 0} \underline{\hat{\hat{w}}}_{, x}\end{array}\right)$

${ }_{0} \underline{\hat{e}}_{y y}={ }_{0} \underline{\hat{e}}_{y z}={ }_{0} \hat{\underline{\hat{e}}}_{z z}=0$

In Eq. (3), the terms, ${ }_{0}^{t} \hat{S}_{x x},{ }_{0}^{t} \hat{S}_{x y},{ }_{0}^{t} \hat{S}_{x z}$, represent three nonzero components of the second PiolaKirchhoff stress tensor for a beam. For large rotation, large displacement, but small strain problems, these stress components can be estimated as [7] 


$$
\begin{aligned}
& {\left[\begin{array}{c}
{ }_{0}^{t} \hat{S}_{x x} \\
{ }_{0}^{t} \hat{S}_{x y} \\
{ }_{0}^{t} \hat{S}_{x z}
\end{array}\right]=\left[\begin{array}{ccc}
E & 0 & 0 \\
0 & 2 k_{s} G & 0 \\
0 & 0 & 2 k_{s} G
\end{array}\right]\left[\begin{array}{c}
{ }_{0}^{t} \hat{\varepsilon}_{x x} \\
{ }_{0}^{t} \hat{\varepsilon}_{x y} \\
{ }_{0}^{t} \hat{\varepsilon}_{x z}
\end{array}\right]} \\
& { }_{0}^{t} \hat{S}_{y y}={ }_{0}^{t} \hat{S}_{z z}={ }_{0}^{t} \hat{S}_{y z}=0
\end{aligned}
$$

where $k_{s}$ represents the shear correction factor for the beam cross-section [7]. The terms $E$ and $G$ represent the elastic modulus and shear modulus of the material. The terms, ${ }_{0}^{t} \hat{\varepsilon}_{x x},{ }_{0}^{t} \hat{\varepsilon}_{x y},{ }_{0}^{t} \hat{\varepsilon}_{x z}$, represent three nonzero components of Green Lagrange strain tensor for a beam which can be defined as [7]

$$
\begin{aligned}
& { }_{0}^{t} \hat{\varepsilon}_{x x}={ }_{0}^{t} \hat{u}_{, x}+\frac{1}{2}\left({ }_{0}^{t} \hat{u}_{, x}^{2}+{ }_{0}^{t} \hat{v}_{, x}^{2}+{ }_{0}^{t} \hat{w}_{, x}{ }^{2}\right) \\
& { }_{0}^{t} \hat{\varepsilon}_{x y}=\frac{1}{2}\left({ }_{0}^{t} \hat{u}_{, y}+{ }_{0}^{t} \hat{v}_{, x}+{ }_{0}^{t} \hat{u}_{, x}{ }_{0}^{t} \hat{u}_{, y}+{ }_{0}^{t} \hat{v}_{, x}{ }_{0}^{t} \hat{v}_{, y}+{ }_{0}^{t} \hat{w}_{, x}{ }_{0}^{t} \hat{w}_{, y}\right) \\
& { }_{0}^{t} \hat{\varepsilon}_{x z}=\frac{1}{2}\left({ }_{0}^{t} \hat{u}_{, z}+{ }_{0}^{t} \hat{w}_{, x}+{ }_{0}^{t} \hat{u}_{, x}{ }_{0}^{t} \hat{u}_{, z}+{ }_{0}^{t} \hat{v}_{, x}{ }_{0}^{t} \hat{v}_{, z}+{ }_{0}^{t} \hat{w}_{, x}{ }_{0}^{t} \hat{w}_{, z}\right) \\
& { }_{0}^{t} \hat{\varepsilon}_{y y}={ }_{0}^{t} \hat{\varepsilon}_{y z}={ }_{0}^{t} \hat{\varepsilon}_{z z}=0
\end{aligned}
$$

In Eq. (3), the second term on the right-hand side is the virtual work associated with the element stress at time $t$. The strain energy density (SED) corresponding to this virtual work for a beam can be calculated as

$W^{N L}={ }_{0}^{t} \hat{S}_{x x} \underline{\hat{e}}_{x x}+2{ }_{0}^{t} \hat{S}_{x y 0} \underline{\hat{e}}_{x y}+2{ }_{0}^{t} \hat{S}_{x z} \underline{\hat{e}}_{x z}$

By using the constitutive relations given in Eq. (5a), the SED given in Eq. (7) can be rewritten as $W^{N L}=E_{0}^{t} \hat{\varepsilon}_{x x} \underline{\hat{e}}_{x x}+4 k_{s} G_{0}{ }_{0} \hat{\varepsilon}_{x y 0} \underline{\hat{e}}_{x y}+4 k_{s} G_{0}{ }^{t} \hat{\varepsilon}_{x z} \underline{\hat{e}}_{x z}$

Moreover, similar to the study in [66], the strain energy density given in Eq. (8) can be averaged through the cross-section of the beam as

$$
\bar{W}^{N L}=\frac{\int_{0_{A}} W^{N L} d\left({ }^{0} A\right)}{{ }^{0} A}=\frac{\int_{0_{A}}\left(E_{0}^{t} \hat{\varepsilon}_{x x} 0 \underline{\hat{e}}_{x x}+4 k_{s} G_{0}^{t} \hat{\varepsilon}_{x y 0} \underline{\hat{e}}_{x y}+4 k_{s} G_{0}^{t} \hat{\varepsilon}_{x z 0} \underline{\hat{e}}_{x z}\right) d\left({ }^{0} A\right)}{{ }^{0} A}
$$

where ${ }^{0} A$ represents the beam cross-sectional area at initial configuration.

In peridynamics, the PD equation of motion can be solved by using an explicit time integration scheme $[12,15,67]$. When the explicit time integration scheme is used, the principle of virtual displacements given in Eq. (3) is used. Therefore, in this study, the SED in classical continuum mechanics and PD for a beam is obtained based on Eq. (9).

\section{Nonlinear beam kinematics in classical continuum mechanics}

In this section, a three-dimensional straight beam is considered as shown in Fig. 2. The beam kinematics are obtained with respect to the body-attached coordinate system of the beam, $x, y, z$, which is called the local coordinate system. The beam is assumed to have a symmetric crosssection. The nonlinear strain energy density for the beam is obtained through three main steps: First, the displacement components of a beam element are presented in Section 3.1. Next, the Green-Lagrange strain and Second Piola-Kirchhoff stress components are presented in Section 3.2 and 3.3, respectively. Finally, the nonlinear strain energy density for a beam structure is presented in Section 3.4. 


\subsection{Displacement components}

Based on the Timoshenko theory [68], a beam has 6 local degrees of freedom, $u, v, w, \theta_{x}, \theta_{y}, \theta_{z}$ as shown in Fig. 2. The terms $u, v, w$ represent the local displacements of the point located at the centre line of the beam and $\theta_{x}, \theta_{y}, \theta_{z}$ represent local rotations of the beam cross-section.

Since in nonlinear analysis, three rotations $\theta_{x}, \theta_{y}, \theta_{z}$ are co-rotated, the rotations of the beam at time $t$ are controlled by using director vectors, ${ }^{t} \mathbf{V}_{s},{ }^{t} \mathbf{V}_{t}$ [7] as shown in Fig. 2.

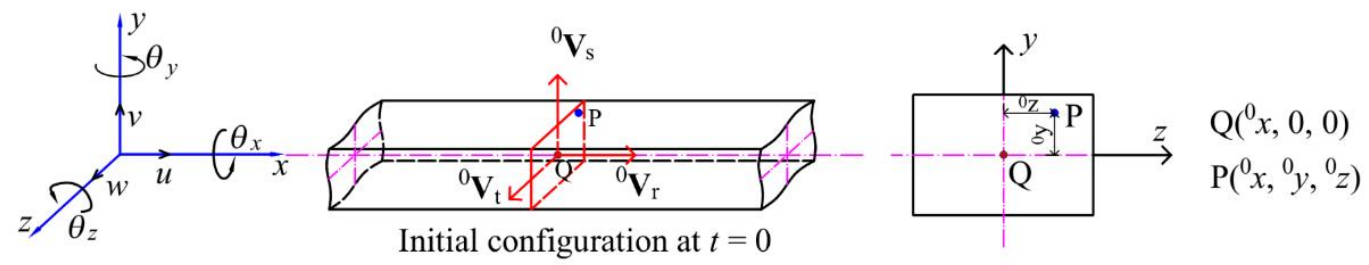

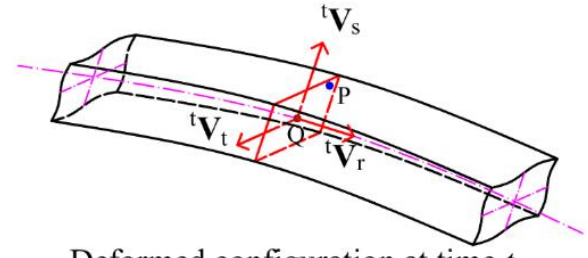

Deformed configuration at time $t$
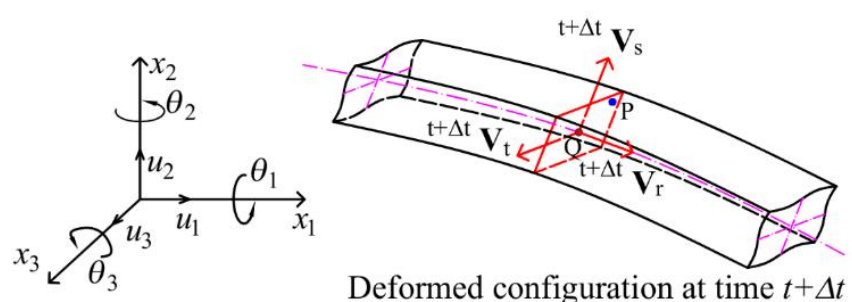

Deformed configuration at time $t+\Delta t$
Displacements of point P: $\left({ }^{t+\Delta t} \widehat{u},{ }^{t+\Delta t} \widehat{v},{ }^{t+\Delta t} \widehat{W}\right)$

Displacements and rotations of point Q: $\left({ }^{\mathrm{t}+\Delta \mathrm{t}} \mathrm{u},{ }^{\mathrm{t}+\Delta \mathrm{t}} \mathrm{v},{ }^{\mathrm{t}+\Delta \mathrm{t}} \mathrm{W},{ }^{\mathrm{t}+\Delta \mathrm{t}} \theta_{x},{ }^{\mathrm{t}+\Delta \mathrm{t}} \theta_{y},{ }^{\mathrm{t}+\Delta \mathrm{t}} \theta_{z}\right)$

Fig. 2. Beam configurations with 6 local DOFs

As shown in Fig. 2, material point $P$ is located at $\left({ }^{0} x,{ }^{0} y,{ }^{0} z\right)$ and material point $Q$ is located at $\left({ }^{0} x, 0,0\right)$ which is on the centre line of the beam.

The degrees of freedom at time $t$ and time $t+\Delta t$ for material points $P$ and $Q$ are represented as $\left({ }^{t} \hat{u},{ }^{t} \hat{v},{ }^{t} \hat{w}\right),\left({ }^{t+\Delta t} \hat{u},{ }^{t+\Delta t} \hat{v},{ }^{t+\Delta t} \hat{w}\right)$ for $P$

$\left({ }^{t} u,{ }^{t} v,{ }^{t} w,{ }^{t} \theta_{x},{ }^{t} \theta_{y},{ }^{t} \theta_{z}\right),\left({ }^{t+\Delta t} u,{ }^{t+\Delta t} v,{ }^{t+\Delta t} w,{ }^{t+\Delta t} \theta_{x},{ }^{t+\Delta t} \theta_{y},{ }^{t+\Delta t} \theta_{z}\right)$ for $Q$

According to Bathe [7], the displacement components of material point $P$ located at $\left({ }^{0} x,{ }^{0} y,{ }^{0} z\right)$ at time $t$ can be defined as

$$
\begin{aligned}
& { }^{t} \hat{u}={ }^{t} u+{ }^{0} z\left({ }^{t} V_{t x}-{ }^{0} V_{t x}\right)+{ }^{0} y\left({ }^{t} V_{s x}-{ }^{0} V_{s x}\right) \\
& { }^{t} \hat{v}={ }^{t} v+{ }^{0} z\left({ }^{t} V_{t y}-{ }^{0} V_{t y}\right)+{ }^{0} y\left({ }^{t} V_{s y}-{ }^{0} V_{s y}\right)
\end{aligned}
$$


${ }^{t} \hat{w}={ }^{t} w+{ }^{0} z\left({ }^{t} V_{t z}-{ }^{0} V_{t z}\right)+{ }^{0} y\left({ }^{t} V_{s z}-{ }^{0} V_{s z}\right)$

where ${ }^{t} V_{s x},{ }^{t} V_{s y},{ }^{t} V_{s z}$ represent components of the director vector ${ }^{t} \mathbf{V}_{s}$ at time $t$ with respect to local axes $x, y, z$, respectively. The terms ${ }^{0} V_{s x},{ }^{0} V_{s y},{ }^{0} V_{s z}$ represent components of the director vector ${ }^{0} \mathbf{V}_{s}$ at time $t=0$ with respect to local axes $x, y, z$, respectively. Similarly, ${ }^{t} V_{t x},{ }^{t} V_{t y},{ }^{t} V_{t z}$ represent components of the director vector ${ }^{t} \mathbf{V}_{t}$ at time $t$ with respect to local axes $x, y, z$, respectively. The terms ${ }^{0} V_{t x},{ }^{0} V_{t y},{ }^{0} V_{t z}$ represent components of the director vector ${ }^{0} \mathbf{V}_{t}$ at time $t=0$ with respect to local axes $x, y, z$, respectively.

Note that, in Eq. (11), the director vectors at time $t$, including $\left({ }^{t} V_{s x},{ }^{t} V_{s y},{ }^{t} V_{s z}\right)$ and $\left({ }^{t} V_{t x},{ }^{t} V_{t y}, V_{t z}\right)$ are unknown. These director vectors can be updated numerically by using the director vectors at the previous time step as given in Eq. (43c-e) in Section 4. Meanwhile, the director vectors at time $t=0$ can be defined based on the initial configuration of the beam. In this section, for simplification, a straight beam is considered, and the beam's local coordinates are assumed to be aligned with the global coordinates as shown in Fig. 2. Therefore, the director vectors at time $t=0$ for a straight beam can be defined as

$$
\begin{aligned}
& { }^{0} \mathbf{V}_{s}=\left[\begin{array}{lll}
0 & 1 & 0
\end{array}\right]^{T} \text { with }{ }^{0} V_{s x}=0 ; \quad{ }^{0} V_{s y}=1 ; \quad{ }^{0} V_{s z}=0 \\
& { }^{0} \mathbf{V}_{t}=\left[\begin{array}{lll}
0 & 0 & 1
\end{array}\right]^{T} \text { with }{ }^{0} V_{t x}=0 ; \quad{ }^{0} V_{t y}=0 ; \quad{ }^{0} V_{t z}=1 \\
& { }^{0} \mathbf{V}_{r}=\left[\begin{array}{lll}
1 & 0 & 0
\end{array}\right]^{T} \text { with }{ }^{0} V_{r x}=1 ; \quad{ }^{0} V_{r y}=0 ; \quad{ }^{0} V_{r z}=0
\end{aligned}
$$

By substituting Eq. (12) into Eq. (11), the displacement components of material point $P$ located at $\left({ }^{0} x,{ }^{0} y,{ }^{0} z\right)$ at time $t$ can be defined as [7]

$$
\begin{aligned}
& { }^{t} \hat{u}={ }^{t} u+{ }^{0} z^{t} V_{t x}+{ }^{0} y^{t} V_{s x} \\
& { }^{t} \hat{v}={ }^{t} v+{ }^{0} z V_{t y}+{ }^{0} y\left({ }^{t} V_{s y}-1\right) \\
& { }^{t} \hat{w}={ }^{t} w+{ }^{0} z\left({ }^{t} V_{t z}-1\right)+{ }^{0} y V_{s z}
\end{aligned}
$$

Similar to Eq. (13), the displacement components of material point $P$ at time $t+\Delta t$ can be calculated as [7]

$$
\begin{aligned}
& { }^{t+\Delta t} \hat{u}={ }^{t+\Delta t} u+{ }^{0} z^{t+\Delta t} V_{t x}+{ }^{0} y^{t+\Delta t} V_{s x} \\
& { }^{t+\Delta t} \hat{v}={ }^{t+\Delta t} v+{ }^{0} z^{t+\Delta t} V_{t y}+{ }^{0} y\left({ }^{t+\Delta t} V_{s y}-1\right) \\
& { }^{t+\Delta t} \hat{w}={ }^{t+\Delta t} w+{ }^{0} z\left({ }^{t+\Delta t} V_{t z}-1\right)+{ }^{0} y^{t+\Delta t} V_{s z}
\end{aligned}
$$

By substituting Eq. (13) and Eq. (14) into Eq. (2), the incremental displacements from time $t$ to time $t+\Delta t$ can be calculated as [7]

$$
\begin{aligned}
& \underline{\hat{u}}={ }^{t+\Delta t} \hat{u}-{ }^{t} \hat{u}={ }^{t+\Delta t} u-{ }^{t} u+{ }^{0} z\left({ }^{t+\Delta t} V_{t x}-{ }^{t} V_{t x}\right)+{ }^{0} y\left({ }^{t+\Delta t} V_{s x}-{ }^{t} V_{s x}\right) \\
& \underline{\hat{v}}={ }^{t+\Delta t} \hat{v}-{ }^{t} \hat{v}={ }^{t+\Delta t} v-{ }^{t} v+{ }^{0} z\left({ }^{t+\Delta t} V_{t y}-{ }^{t} V_{t y}\right)+{ }^{0} y\left({ }^{t+\Delta t} V_{s y}-{ }^{t} V_{s y}\right) \\
& \hat{\hat{w}}={ }^{t+\Delta t} \hat{w}-{ }^{t} \hat{w}={ }^{t+\Delta t} w-{ }^{t} w+{ }^{0} z\left({ }^{t+\Delta t} V_{t z}-{ }^{t} V_{t z}\right)+{ }^{0} y\left({ }^{t+\Delta t} V_{s z}-{ }^{t} V_{s z}\right)
\end{aligned}
$$


The relations given in Eq. (15) can be rewritten as

$$
\begin{aligned}
& \underline{\hat{u}}=\underline{u}+{ }^{0} z \underline{V}_{t x}+{ }^{0} y \underline{V}_{s x} \\
& \underline{\hat{v}}=\underline{v}+{ }^{0} z \underline{V}_{t y}+{ }^{0} y \underline{V}_{s y} \\
& \underline{\hat{w}}=\underline{w}+{ }^{0} z \underline{V}_{t z}+{ }^{0} y \underline{V}_{s z}
\end{aligned}
$$

with

$$
\underline{u}={ }^{t+\Delta t} u-{ }^{t} u
$$$$
\underline{v}={ }^{t+\Delta t} v-{ }^{t} v
$$$$
\underline{w}={ }^{t+\Delta t} w-{ }^{t} w
$$

and

$\underline{V}_{s x}={ }^{t+\Delta t} V_{s x}-{ }^{t} V_{s x} ; \quad \underline{V}_{t x}={ }^{t+\Delta t} V_{t x}-{ }^{t} V_{t x}$

$\underline{V}_{s y}={ }^{t+\Delta t} V_{s y}-{ }^{t} V_{s y} ; \quad \underline{V}_{t y}={ }^{t+\Delta t} V_{t y}-{ }^{t} V_{t y}$

$\underline{V}_{s z}={ }^{t+\Delta t} V_{s z}-V_{s z} ; \quad \underline{V}_{t z}={ }^{t+\Delta t} V_{t z}-{ }^{t} V_{t z}$

In Eq. (16d-f), $\underline{u}, \underline{v}, \underline{w}$ represent the incremental displacements from time $t$ to time $t+\Delta t$ of point $Q$ located at $\left({ }^{0} x, 0,0\right)$ on the centre line of the beam. The terms $\underline{V}_{s x}, \underline{V}_{s y}, \underline{V}_{s z}$ represent components of the incremental director vector, $\underline{\mathbf{V}}_{s}$, from time $t$ to time $t+\Delta t$. Similarly, the terms $\underline{V}_{t x}, \underline{V}_{t y}, \underline{V}_{t z}$ represent components of the incremental director vector, $\underline{\mathbf{V}}_{t}$, from time $t$ to time $t+\Delta t$.

The relations given in Eq. (16g-i) can be rewritten in the compact form as

$$
\begin{aligned}
& \underline{\mathbf{V}}_{s}={ }^{t+\Delta t} \mathbf{V}_{s}-{ }^{t} \mathbf{V}_{s} \\
& \underline{\mathbf{V}}_{t}={ }^{t+\Delta t} \mathbf{V}_{t}-{ }^{t} \mathbf{V}_{t}
\end{aligned}
$$

with

$$
\begin{aligned}
& \underline{\mathbf{V}}_{s}=\left[\begin{array}{lll}
\underline{V}_{s x} & \underline{V}_{s y} & \underline{V}_{s z}
\end{array}\right] \\
& \underline{\mathbf{V}}_{t}=\left[\begin{array}{lll}
\underline{V}_{t x} & \underline{V}_{t y} & \underline{V}_{t z}
\end{array}\right]
\end{aligned}
$$

According to Bathe [7], the incremental director vectors given in Eq. (17c-d) can be approximated by using the director vectors at time $t$ as

$$
\begin{aligned}
& \underline{\mathbf{V}}_{s}=\underline{\boldsymbol{\theta}} \times{ }^{t} \mathbf{V}_{s}=\left[\begin{array}{llll}
\left(\underline{\theta}_{y}{ }^{t} V_{s z}-\underline{\theta}_{z}{ }^{t} V_{s y}\right) & \left(\underline{\theta}_{z}{ }^{t} V_{s x}-\underline{\theta}_{x}{ }^{t} V_{s z}\right) & \left(\underline{\theta}_{x}{ }^{t} V_{s y}-\underline{\theta}_{y}{ }^{t} V_{s x}\right)
\end{array}\right]^{T} \\
& \underline{\mathbf{V}}_{t}=\underline{\boldsymbol{\theta}} \times{ }^{t} \mathbf{V}_{t}=\left[\begin{array}{llll}
\left.\underline{\theta}_{y}{ }^{t} V_{t z}-\underline{\theta}_{z}{ }^{t} V_{t y}\right) & \left(\underline{\theta}_{z}{ }^{t} V_{t x}-\underline{\theta}_{x}{ }^{t} V_{t z}\right) & \left(\underline{\theta}_{x}{ }^{t} V_{t y}-\underline{\theta}_{y}{ }^{t} V_{t x}\right)
\end{array}\right]^{T}
\end{aligned}
$$

with

$$
\underline{\boldsymbol{\theta}}={ }^{t+\Delta t} \boldsymbol{\theta}-{ }^{t} \boldsymbol{\theta}=\left[\begin{array}{lll}
\underline{\theta}_{x} & \underline{\theta}_{y} & \underline{\theta}_{z}
\end{array}\right]^{T}
$$

and

$$
\begin{aligned}
& \underline{\theta}_{x}={ }^{t+\Delta t} \theta_{x}-{ }^{t} \theta_{x} \\
& \underline{\theta}_{y}={ }^{t+\Delta t} \theta_{y}-{ }^{t} \theta_{y} \\
& \underline{\theta}_{z}={ }^{t+\Delta t} \theta_{z}-{ }^{t} \theta_{z}
\end{aligned}
$$


where $\underline{\theta}_{x}, \underline{\theta}_{y}, \underline{\theta}_{z}$ represent the incremental rotations of the beam cross-section from time $t$ to time $t+\Delta t$.

Therefore, by using the incremental director vectors given in Eq. (18a-b), the incremental displacements in Eq. (16a-c) can be calculated as

$$
\begin{aligned}
& \underline{\hat{u}}=\underline{u}+{ }^{0} z\left(\underline{\theta}_{y}{ }^{t} V_{t z}-\underline{\theta}_{z}{ }^{t} V_{t y}\right)+{ }^{0} y\left(\underline{\theta}_{y}{ }^{t} V_{s z}-\underline{\theta}_{z}{ }^{t} V_{s y}\right) \\
& \underline{\hat{v}}=\underline{v}+{ }^{0} z\left(\underline{\theta}_{z}{ }^{t} V_{t x}-\underline{\theta}_{x}{ }^{t} V_{t z}\right)+{ }^{0} y\left(\underline{\theta}_{z}{ }^{t} V_{s x}-\underline{\theta}_{x}{ }^{t} V_{s z}\right) \\
& \hat{\hat{w}}=\underline{w}+{ }^{0} z\left(\underline{\theta}_{x}{ }^{t} V_{t y}-\underline{\theta}_{y}{ }^{t} V_{t x}\right)+{ }^{0} y\left(\underline{\theta}_{x}{ }^{t} V_{s y}-\underline{\theta}_{y}{ }^{t} V_{s x}\right)
\end{aligned}
$$

\subsection{Strain components}

By using displacements at time $t$ given in Eq. (13), the Green-Lagrange strain components in Eq. (6) can be calculated as

$$
\begin{aligned}
& { }_{0}^{t} \hat{\varepsilon}_{x x}={ }_{0}^{t} u_{, x}+\frac{1}{2}\left\{\left({ }_{0}^{t} u_{, x}\right)^{2}+\left({ }_{0}^{t} v_{, x}\right)^{2}+\left({ }_{0}^{t} w_{, x}\right)^{2}\right\} \\
& +{ }^{0} y\left\{{ }_{0}^{t} V_{s x, x}+\left({ }_{0}^{t} u_{, x}\right)\left({ }_{0}^{t} V_{s x, x}\right)+\left({ }_{0}^{t} v_{, x}\right)\left({ }_{0}^{t} V_{s y, x}\right)+\left({ }_{0}^{t} w_{, x}\right)\left({ }_{0}^{t} V_{s z, x}\right)\right\} \\
& +{ }^{0} z\left\{{ }_{0}^{t} V_{t x, x}+\left({ }_{0}^{t} u_{, x}\right)\left({ }_{0}^{t} V_{t x, x}\right)+\left({ }_{0}^{t} v_{, x}\right)\left({ }_{0}^{t} V_{t y, x}\right)+\left({ }_{0}^{t} w_{, x}\right)\left({ }_{0}^{t} V_{t, x}\right)\right\}
\end{aligned}
$$

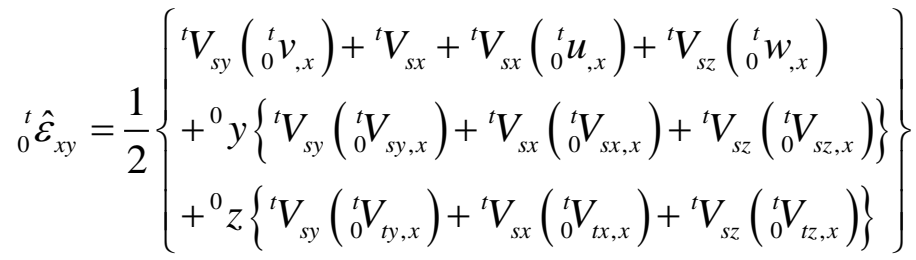

$$
\begin{aligned}
& { }_{0}^{t} \hat{\varepsilon}_{x z}=\frac{1}{2}\left\{\begin{array}{l}
{ }^{t} V_{t z}\left({ }_{0}^{t} w_{, x}\right)+{ }^{t} V_{t x}+{ }^{t} V_{t x}\left({ }_{0}^{t} u_{, x}\right)+{ }^{t} V_{t y}\left({ }_{0}^{t} V_{, x}\right) \\
+{ }^{0} y\left\{{ }_{t z} V_{t z}\left({ }_{0}^{t} V_{s z, x}\right)+{ }^{t} V_{t x}\left({ }_{0}^{t} V_{s x, x}\right)+{ }^{t} V_{t y}\left({ }_{0}^{t} V_{s y, x}\right)\right\} \\
+{ }^{0} z\left\{{ }^{t} V_{t z}\left({ }_{0}^{t} V_{t z, x}\right)+{ }^{t} V_{t x}\left({ }_{0}^{t} V_{t x, x}\right)+{ }^{t} V_{t y}\left({ }_{0}^{t} V_{t y, x}\right)\right\}
\end{array}\right\}
\end{aligned}
$$

with

$$
\begin{array}{lll}
{ }_{0}^{t} u_{, x}=\frac{\partial^{t} u}{\partial^{0} x} ; & { }_{0}^{t} V_{, x}=\frac{\partial^{t} v}{\partial^{0} x} ; & { }_{0}^{t} w_{, x}=\frac{\partial^{t} w}{\partial^{0} x} \\
{ }_{0}^{t} V_{s x, x}=\frac{\partial^{t} V_{s x}}{\partial^{0} x} ; & { }_{0}^{t} V_{s y, x}=\frac{\partial^{t} V_{s y}}{\partial^{0} x} ; & { }_{0}^{t} V_{s z, x}=\frac{\partial^{t} V_{s z}}{\partial^{0} x} \\
{ }_{0}^{t} V_{t x, x}=\frac{\partial^{t} V_{t x}}{\partial^{0} x} ; & { }_{0}^{t} V_{t y, x}=\frac{\partial^{t} V_{t y}}{\partial^{0} x} ; & { }_{0}^{t} V_{t z, x}=\frac{\partial^{t} V_{t z}}{\partial^{0} x}
\end{array}
$$

By using the displacements at time $t$ given in Eq. (13) and the incremental displacements given in Eq. (19), the linear incremental strain components given in Eq. (4a-c) can be calculated as 


$$
\begin{aligned}
& { }_{0} \underline{\hat{e}}_{x x}={ }_{0} \underline{u}_{, x}\left(1+{ }_{0}^{t} u_{, x}\right)+\left({ }_{0}^{t} v_{, x}\right)\left({ }_{0} \underline{v}_{, x}\right)+\left({ }_{0}^{t} w_{, x}\right)\left({ }_{0} \underline{w}_{, x}\right) \\
& +{ }^{0} y\left\{\begin{array}{l}
\left(1+{ }_{0}^{t} u_{, x}\right) \frac{\partial\left(\underline{\theta}_{y}{ }^{t} V_{s z}-\underline{\theta}_{z}{ }^{t} V_{s y}\right)}{\partial^{0} x}+\left({ }_{0}^{t} V_{s x, x}\right)\left({ }_{0} \underline{u}_{, x}\right) \\
+\left({ }_{0}^{t} V_{s y, x}\right)\left({ }_{0} \underline{v}_{, x}\right)+{ }_{0}^{t} v_{, x} \frac{\partial\left(\underline{\theta}_{z}{ }^{t} V_{s x}-\underline{\theta}_{x}{ }^{t} V_{s z}\right)}{\partial^{0} x}+\left({ }_{0}^{t} V_{s z, x}\right)\left({ }_{0} \underline{w}_{, x}\right)+{ }_{0}^{t} w_{, x} \frac{\partial\left(\underline{\theta}_{x}{ }^{t} V_{s y}-\underline{\theta}_{y}{ }^{t} V_{s x}\right)}{\partial^{0} x}
\end{array}\right\} \\
& +{ }^{0} z\left\{\begin{array}{l}
\left(1+{ }_{0}^{t} u_{, x}\right) \frac{\partial\left(\underline{\theta}_{y}{ }^{t} V_{t z}-\underline{\theta}_{z}{ }^{t} V_{t y}\right)}{\partial^{0} x}+\left({ }_{0}^{t} V_{t x, x}\right)\left({ }_{0} u_{, x}\right) \\
+\left({ }_{0}^{t} V_{t y, x}\right)\left({ }_{0} v_{, x}\right)+{ }_{0}^{t} v_{, x} \frac{\partial\left(\underline{\theta}_{z}{ }^{t} V_{t x}-\underline{\theta}_{x}{ }^{t} V_{t z}\right)}{\partial^{0} x}+\left({ }_{0}^{t} V_{t z, x}\right)\left({ }_{0} w_{, x}\right)+{ }_{0}^{t} w_{, x} \frac{\partial\left(\underline{\theta}_{x}{ }^{t} V_{t y}-\underline{\theta}_{y}{ }^{t} V_{t x}\right)}{\partial{ }^{0} x}
\end{array}\right\} \\
& { }_{0} \underline{\hat{e}}_{x y}=\frac{1}{2}\left(\begin{array}{l}
{ }^{t} V_{s y}\left({ }_{0} \underline{v}_{, x}\right)+{ }^{t} V_{s x}\left({ }_{0} \underline{u}_{, x}\right)+{ }^{t} V_{s z}\left({ }_{0} \underline{w}_{, x}\right)+\left(1+{ }_{0}^{t} u_{, x}\right)\left(\underline{\theta}_{y}{ }^{t} V_{s z}-\underline{\theta}_{z}{ }^{t} V_{s y}\right) \\
+\left(\underline{\theta}_{z}{ }^{t} V_{s x}-\underline{\theta}_{x}{ }^{t} V_{s z}\right){ }_{0}^{t} V_{, x}+{ }_{0}^{t} w_{, x}\left(\underline{\theta}_{x}{ }^{t} V_{s y}-\underline{\theta}_{y}{ }^{t} V_{s x}\right) \\
+{ }^{0} z\left\{{ }_{0} \underline{\theta}_{x, x}\left({ }^{t} V_{t y}{ }^{t} V_{s z}-{ }^{t} V_{t z}{ }^{t} V_{s y}\right)+{ }_{0} \underline{\theta}_{y, x}\left({ }^{t} V_{t z}{ }^{t} V_{s x}-{ }^{t} V_{t x}{ }^{t} V_{s z}\right)+{ }_{0} \underline{\theta}_{z, x}\left({ }^{t} V_{t x}{ }^{t} V_{s y}-{ }^{t} V_{t y}{ }^{t} V_{s x}\right)\right\}
\end{array}\right) \\
& { }_{0} \underline{\hat{e}}_{x z}=\frac{1}{2}\left(\begin{array}{l}
{ }^{t} V_{t z}\left({ }_{0} \underline{w}_{, x}\right)+{ }^{t} V_{t x}\left({ }_{0} \underline{u}_{, x}\right)+{ }^{t} V_{t y}\left({ }_{0} \underline{v}_{, x}\right)+\left(1+{ }_{0}^{t} u_{, x}\right)\left(\underline{\theta}_{y}{ }^{t} V_{t z}-\underline{\theta}_{z}{ }^{t} V_{t y}\right) \\
+{ }_{0}^{t} v_{, x}\left(\underline{\theta}_{z}{ }^{t} V_{t x}-\underline{\theta}_{x}{ }^{t} V_{t z}\right)+{ }_{0}^{t} w_{, x}\left(\underline{\theta}_{x}{ }^{t} V_{t y}-\underline{\theta}_{y}{ }^{t} V_{t x}\right) \\
+{ }^{0} y\left\{{ }_{0} \underline{\theta}_{x, x}\left({ }^{t} V_{s y}{ }^{t} V_{t z}-{ }^{t} V_{s z}{ }^{t} V_{t y}\right)+{ }_{0} \underline{\theta}_{y, x}\left({ }^{t} V_{s z}{ }^{t} V_{t x}-{ }^{t} V_{s x}{ }^{t} V_{t z}\right)+{ }_{0} \underline{\theta}_{z, x}\left({ }^{t} V_{s x}{ }^{t} V_{t y}-{ }^{t} V_{s y}{ }^{t} V_{t x}\right)\right\}
\end{array}\right)
\end{aligned}
$$

with

${ }_{0} \underline{u}_{, x}=\frac{\partial \underline{u}}{\partial^{0} x} ; \quad{ }_{0} \underline{v}_{, x}=\frac{\partial \underline{v}}{\partial^{0} x} ; \quad 0 \underline{w}_{, x}=\frac{\partial \underline{w}}{\partial^{0} x}$

\subsection{Strain energy density}

By substituting the Green-Lagrange strain components given in Eq. (20a-c) and the incremental strain components given in Eq. (21a-c) into Eq. (9), the averaged strain energy density through the cross-section of the beam of a beam can be represented as

$\bar{W}^{N L}=\bar{W}_{\text {axial }}^{N L}+\bar{W}_{\text {bending }}^{N L}+\bar{W}_{\text {shear }}^{N L}+\bar{W}_{\text {torsion }}^{N L}$

with

$$
\begin{aligned}
& \bar{W}_{\text {axial }}^{N L}=E\left({ }_{0}^{t} a_{10}\right)\left({ }_{0} \underline{a}_{10}\right) \\
& \bar{W}_{\text {bending }}^{N L}=\frac{E I_{y y}}{{ }^{0} A}\left({ }_{0}^{t} a_{12}\right)\left({ }_{0} \underline{a}_{12}\right)+\frac{E I_{z z}}{{ }^{0} A}\left({ }_{0}^{t} a_{11}\right)\left({ }_{0} \underline{a}_{11}\right) \\
& \bar{W}_{\text {shear }}^{N L}=k_{s} G\left[\left({ }_{0}^{t} a_{13}\right)\left({ }_{0} \underline{a}_{13}\right)+\left({ }_{0}^{t} a_{14}\right)\left({ }_{0} \underline{a}_{14}\right)\right] \\
& \bar{W}_{\text {torsion }}^{N L}=\frac{k_{t} G}{{ }^{0} A}\left({ }_{0}^{t} a_{15}\right)\left({ }_{0} \underline{a}_{15}\right)
\end{aligned}
$$

and 


$$
\begin{aligned}
& { }_{0}^{t} a_{1}={ }_{0}^{t} u_{, x} ; \quad{ }_{0}^{t} a_{2}={ }_{0}^{t} v_{, x} ; \quad{ }_{0}^{t} a_{3}={ }_{0}^{t} w_{, x} \\
& { }_{0}^{t} a_{4}={ }_{0}^{t} V_{s x, x} ; \quad{ }_{0}^{t} a_{5}={ }_{0}^{t} V_{s y, x} ; \quad{ }_{0}^{t} a_{6}={ }_{0}^{t} V_{s z, x} \\
& { }_{0}^{t} a_{7}={ }_{0}^{t} V_{t x, x} ; \quad{ }_{0}^{t} a_{8}={ }_{0}^{t} V_{t y, x} ; \quad{ }_{0}^{t} a_{9}={ }_{0}^{t} V_{t z, x} \\
& { }_{0}^{t} a_{10}={ }_{0}^{t} a_{1}+\frac{1}{2}\left({ }_{0}^{t} a_{1}^{2}+{ }_{0}^{t} a_{2}^{3}+{ }_{0}^{t} a_{3}^{2}\right) \\
& { }_{0}^{t} a_{11}={ }_{0}^{t} a_{4}+\left({ }_{0}^{t} a_{1}\right)\left({ }_{0}^{t} a_{4}\right)+\left({ }_{0}^{t} a_{2}\right)\left({ }_{0}^{t} a_{5}\right)+\left({ }_{0}^{t} a_{3}\right)\left({ }_{0}^{t} a_{6}\right) \\
& { }_{0}^{t} a_{12}={ }_{0}^{t} a_{7}+{ }_{0}^{t} a_{10}{ }^{t} a_{7}+{ }_{0}^{t} a_{20}{ }_{0}^{t} a_{8}+{ }_{0}^{t} a_{30}{ }_{0}^{t} a_{9} \\
& { }_{0}^{t} a_{13}={ }^{t} V_{s y}\left({ }_{0}^{t} a_{2}\right)+{ }^{t} V_{s x}+{ }^{t} V_{s x}\left({ }_{0}^{t} a_{1}\right)+{ }^{t} V_{s z}\left({ }_{0}^{t} a_{3}\right) \\
& { }_{0}^{t} a_{14}={ }^{t} V_{t z}\left({ }_{0}^{t} a_{3}\right)+{ }^{t} V_{t x}+{ }^{t} V_{t x}\left({ }_{0}^{t} a_{1}\right)+{ }^{t} V_{t y}\left({ }_{0}^{t} a_{2}\right) \\
& { }_{0}^{t} a_{15}={ }^{t} V_{s y}\left({ }_{0}^{t} a_{8}\right)+{ }^{t} V_{s x}\left({ }_{0}^{t} a_{7}\right)+{ }^{t} V_{s z}\left({ }_{0}^{t} a_{9}\right) \\
& { }_{0} \underline{a}_{10}=\left({ }_{0} \underline{u}_{, x}\right)\left(1+{ }_{0}^{t} a_{1}\right)+{ }_{0}^{t} a_{2}\left({ }_{0} \underline{v}_{x}\right)+{ }_{0}^{t} a_{3}\left({ }_{0} \underline{w}_{, x}\right) \\
& { }_{0} \underline{a}_{11}={ }_{0}^{t} a_{4}\left({ }_{0} \underline{u}_{, x}\right)+{ }_{0}^{t} a_{5}\left({ }_{0} \underline{v}_{, x}\right)+{ }_{0}^{t} a_{6}\left({ }_{0} \underline{w}_{, x}\right)+\left(1+{ }_{0}^{t} a_{1}\right) \frac{\partial\left(\underline{\theta}_{y}{ }^{t} V_{s z}-\underline{\theta}_{z}{ }^{t} V_{s y}\right)}{\partial^{0} x}+ \\
& +{ }_{0}^{t} a_{2} \frac{\partial\left(\underline{\theta}_{z}{ }^{t} V_{s x}-\underline{\theta}_{x}{ }^{t} V_{s z}\right)}{\partial^{0} x}+{ }_{0}^{t} a_{3} \frac{\partial\left(\underline{\theta}_{x}{ }^{t} V_{s y}-\underline{\theta}_{y}{ }^{t} V_{s x}\right)}{\partial^{0} x} \\
& { }_{0} \underline{a}_{12}=\left({ }_{0}^{t} V_{t x, x}\right)\left({ }_{0} u_{, x}\right)+{ }_{0}^{t} a_{8}\left({ }_{0} v_{, x}\right)+{ }_{0}^{t} a_{9}\left({ }_{0} w_{, x}\right)+\left(1+{ }_{0}^{t} a_{1}\right) \frac{\partial\left(\underline{\theta}_{y}{ }^{t} V_{t z}-\underline{\theta}_{z}{ }^{t} V_{t y}\right)}{\partial^{0} x}+ \\
& +{ }_{0}^{t} a_{2} \frac{\partial\left(\underline{\theta}_{z}{ }^{t} V_{t x}-\underline{\theta}_{x}{ }^{t} V_{t z}\right)}{\partial^{0} x}+{ }_{0}^{t} a_{3} \frac{\partial\left(\underline{\theta}_{x}{ }^{t} V_{t y}-\underline{\theta}_{y}{ }^{t} V_{t x}\right)}{\partial^{0} x} \\
& { }_{0} \underline{a}_{13}={ }^{t} V_{s x}\left({ }_{0} \underline{u}_{, x}\right)+{ }^{t} V_{s y}\left({ }_{0} \underline{v}_{, x}\right)+{ }^{t} V_{s z}\left({ }_{0} \underline{w}_{x}\right)+\left(1+{ }_{0}^{t} a_{1}\right)\left(\underline{\theta}_{y}{ }^{t} V_{s z}-\underline{\theta}_{z}{ }^{t} V_{s y}\right) \\
& +{ }_{0}^{t} a_{2}\left(\underline{\theta}_{z}{ }^{t} V_{s x}-\underline{\theta}_{x}{ }^{t} V_{s z}\right)+{ }_{0}^{t} a_{3}\left(\underline{\theta}_{x}{ }^{t} V_{s y}-\underline{\theta}_{y}{ }^{t} V_{s x}\right) \\
& { }_{0} \underline{a}_{14}={ }^{t} V_{t z}\left({ }_{0} \underline{w}_{x}\right)+{ }^{t} V_{t x}\left({ }_{0} \underline{u}_{, x}\right)+{ }^{t} V_{t y}\left({ }_{0} \underline{v}_{, x}\right)+\left(1+{ }_{0}^{t} a_{1}\right)\left(\underline{\theta}_{y}{ }^{t} V_{t z}-\underline{\theta}_{z}{ }^{t} V_{t y}\right) \\
& +{ }_{0}^{t} a_{2}\left(\underline{\theta}_{z}{ }^{t} V_{t x}-\underline{\theta}_{x}{ }^{t} V_{t z}\right)+{ }_{0}^{t} a_{3}\left(\underline{\theta}_{x}{ }^{t} V_{t y}-\underline{\theta}_{y}{ }^{t} V_{t x}\right) \\
& { }_{0} \underline{a}_{15}={ }_{0} \underline{\theta}_{x, x}\left({ }^{t} V_{t y}{ }^{t} V_{s z}-{ }^{t} V_{t z}{ }^{t} V_{s y}\right)+{ }_{0} \underline{\theta}_{y, x}\left({ }^{t} V_{t z}{ }^{t} V_{s x}-{ }^{t} V_{t x}{ }^{t} V_{s z}\right)+{ }_{0} \underline{\theta}_{z, x}\left({ }^{t} V_{t x}{ }^{t} V_{s y}-{ }^{t} V_{t y}{ }^{t} V_{s x}\right)
\end{aligned}
$$

where $\bar{W}_{\text {axial }}^{N L}, \bar{W}_{\text {bending }}^{N L}, \bar{W}_{\text {shear }}^{N L}$ and $\bar{W}_{\text {torsion }}^{N L}$ represent strain energy density corresponding to axial, bending, shear, and torsional deformations, respectively. The terms $I_{y y}$ and $I_{z z}$ given in Eq. (22c) represent the area moment of inertia of beam cross-section. The term $k_{s}$ in Eq. (22d) represents the shear correction factor for the beam cross-section [7]. The term $k_{t}$ in Eq. (22e) represents the torsional constant for beam cross-section which can be defined as $[66,69]$

$k_{t}=\int_{A}\left\{\left({ }^{0} z\right)^{2}+\left({ }^{0} y\right)^{2}\right\} d\left({ }^{0} A\right)$

\section{Nonlinear beam kinematics in peridynamics}

Peridynamics is a new nonlocal theory using integro-differential equations. In peridynamics, each material point in peridynamics has interactions with surrounding material points located within a 
horizon $H_{x}$ with a radius of $\delta$ which is called horizon size as shown in Fig. 3. Material points within the horizon, $H_{x}$ are called family members of that material point $[11,12,14,15]$. The original PD equation of motion is proposed by Silling [11], [12]. For nonlinear analysis of a beam, the PD equation of motion for material point $k$ can be described as

$\rho\left({ }^{0} \mathbf{x}\right)\left({ }^{t} \ddot{\mathbf{u}}\left({ }^{0} \mathbf{x}, t\right)\right)=\int_{H_{\mathrm{x}}}\left(\begin{array}{c}{ }_{0}^{t} \mathbf{t}\left({ }^{t} \mathbf{u}^{\prime}-{ }^{t} \mathbf{u},{ }^{0} \mathbf{x}^{\prime}-{ }^{0} \mathbf{x}, t\right) \\ -{ }_{0}^{t} \mathbf{t}^{\prime}\left({ }^{t} \mathbf{u}-{ }^{t} \mathbf{u}^{\prime},{ }^{0} \mathbf{x}-{ }^{0} \mathbf{x}^{\prime}, t\right)\end{array}\right) d^{0} V^{\prime}+{ }^{t} \mathbf{b}\left({ }^{0} \mathbf{x}, t\right)$

which can also be represented in discrete form as

$\rho_{(k)}\left({ }^{t} \ddot{\mathbf{u}}_{(k)}\right)=\sum_{j=1}^{N}\left(\begin{array}{c}{ }_{0}^{t} \mathbf{t}_{(k)(j)}\left({ }^{t} \mathbf{u}_{(j)}-{ }^{t} \mathbf{u}_{(k)},{ }^{0} \mathbf{x}_{(j)}-{ }^{0} \mathbf{x}_{(k)}, t\right) \\ -{ }_{0}^{t} \mathbf{t}_{(j)(k)}\left({ }^{t} \mathbf{u}_{(k)}-{ }^{t} \mathbf{u}_{(j)},{ }^{0} \mathbf{x}_{(k)}-{ }^{0} \mathbf{x}_{(j)}, t\right)\end{array}\right){ }^{0} V_{(j)}+{ }^{t} \mathbf{b}_{(k)}$

where $\rho$ represents the mass density, ${ }^{t} \ddot{\mathbf{u}}$ represents the vector of accelerations at time $t$. The term ${ }^{t} \mathbf{u}$ represents the vector of displacements at time $t,{ }^{t} \mathbf{b}_{(k)}$ represents the vector of external forces and moments per unit volume at time $t$. In Eq. (24b), $N$ represents the number of family members of material point $k$, and $j$ represents a family member material point $k$. The term ${ }_{0}^{t} \mathbf{t}_{(k)(j)}$ represents the force density at time $t$ that material point $j$ exerts on material point $k$, and ${ }_{0}^{t} \mathbf{t}_{(j)(k)}$ represents the force density at time $t$ that material point $k$ exerts on point $j$. For a beam with six degrees of freedom, the parameters in Eq. (24) can be represented as

$$
\begin{aligned}
& { }_{0}^{t} \mathbf{t}_{(k)(j)}=\left[\begin{array}{llllll}
{ }_{0}^{t} t_{(k)(j)}^{u} & { }_{0}^{t} t_{(k)(j)}^{v} & { }_{0}^{t} t_{(k)(j)}^{w} & { }_{0}^{t} t_{(k)(j)}^{\theta_{x}} & { }_{0}^{t} t_{(k)(j)}^{\theta_{y}} & { }_{0}^{t} t_{(k)(j)}^{\theta_{z}}
\end{array}\right]^{T} \\
& { }_{0}^{t} \mathbf{t}_{(j)(k)}=\left[\begin{array}{llllll}
{ }_{0}^{t} t_{(k)(j)(k)}^{u} & { }_{0}^{t} t_{(j)(k)}^{v} & { }_{0}^{t} t_{(j)(k)}^{w} & { }_{0}^{t} t_{(j)(k)}^{\theta_{x}} & { }_{0}^{t} t_{(j)(k)}^{\theta_{y}} & { }_{0}^{t} t_{(j)(k)}^{\theta_{z}}
\end{array}\right]^{T} \\
& { }^{t} \ddot{\mathbf{u}}_{(k)}=\left[\begin{array}{llllll}
{ }^{t} \ddot{u}_{(k)} & { }^{t} \ddot{v}_{(k)} & { }^{t} \ddot{w}_{(k)} & { }^{t} \ddot{\theta}_{x(k)} & { }^{t} \ddot{\theta}_{y(k)} & { }^{t} \ddot{\theta}_{z(k)}
\end{array}\right]^{T} \\
& { }^{t} \mathbf{b}_{(k)}=\left[\begin{array}{llllll}
{ }^{t} b_{x(k)} & { }^{t} b_{y(k)} & { }^{t} b_{z(k)} & { }^{t} m_{x(k)} & { }^{t} m_{y(k)} & { }^{t} m_{z(k)}
\end{array}\right]^{T} \\
& { }^{0} \mathbf{x}_{(k)}=\left[\begin{array}{lll}
{ }^{0} x_{(k)} & 0 & 0
\end{array}\right] \\
& { }^{t} \mathbf{u}_{(k)}=\left[\begin{array}{llllll}
{ }^{t} u_{(k)} & { }^{t} v_{(k)} & { }^{t} w_{(k)} & { }^{t} \theta_{x(k)} & { }^{t} \theta_{y(k)} & { }^{t} \theta_{z(k)}
\end{array}\right]^{T}
\end{aligned}
$$

where ${ }_{0}^{t} t_{(k)(j)}^{u},{ }_{0}^{t} t_{(k)(j)}^{v},{ }_{0}^{t} t_{(k)(j)}^{w},{ }_{0}^{t} t_{(k)(j)}^{\theta_{x}},{ }_{0}^{t} t_{(k)(j)}^{\theta_{y}}$, and ${ }_{0}^{t} t_{(k)(j)}^{\theta_{z}}$ represents force densities corresponding to six degrees of freedom, $u, v, w, \theta_{x}, \theta_{y}$, and $\theta_{z}$, respectively. 


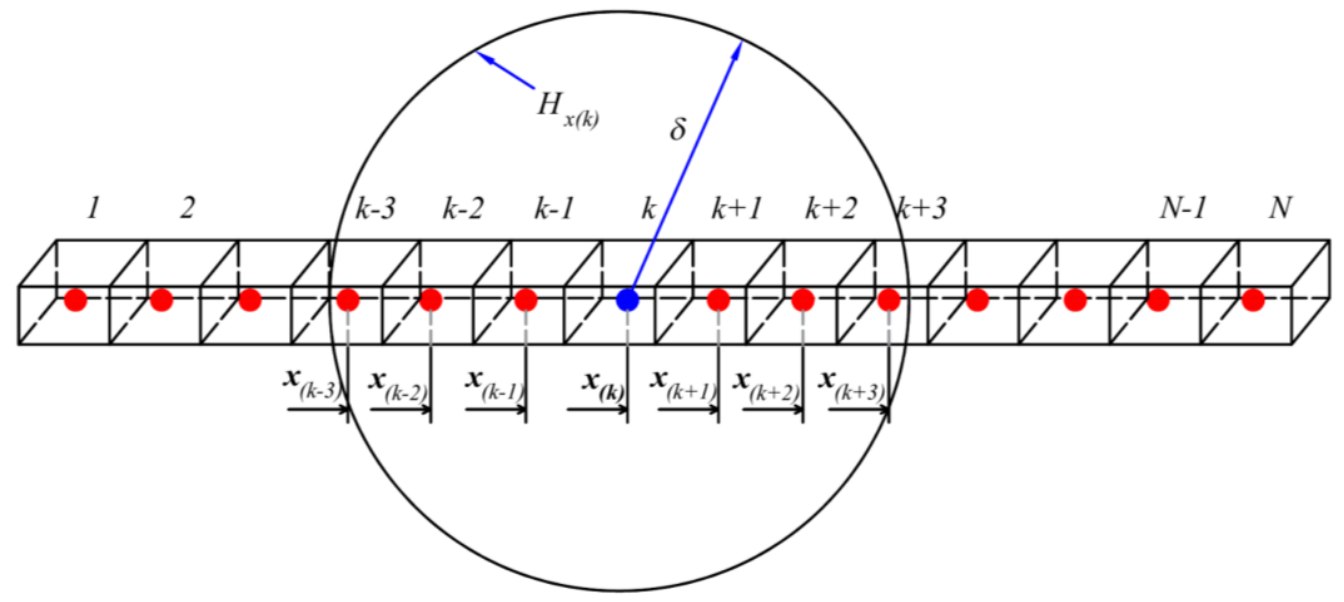

Fig. 3. PD material points and horizon size for a beam

According to Madenci and Oterkus [15], the PD equation of motion can be derived by satisfying the Euler-Lagrange equation. The relationship between the components of force densities and strain energy density can be described as $[15,21]$

${ }_{0}^{t} t_{(k)(j)}^{q_{i}}=-\frac{1}{{ }^{0} V_{(j)}} \frac{\partial \bar{W}_{(k)}^{N L P D}}{\partial \underline{q}_{i(k)}}$

${ }_{0}^{t} t_{(j)(k)}^{q_{i}}=-\frac{1}{{ }^{0} V_{(k)}} \frac{\partial \bar{W}_{(j)}^{N L P D}}{\partial \underline{q}_{i(j)}}$

with

$q_{i}=u, v, w, \theta_{x}, \theta_{y}, \theta_{z}$

$\underline{q}_{i}=\underline{u}, \underline{v}, \underline{w}, \underline{\theta}_{x}, \underline{\theta}_{y}, \underline{\theta}_{z}$

where the parameter $q_{i}$ represents the degree of freedom which can be $u, v, w, \theta_{x}$, $\theta_{y}$, or $\theta_{z}$. The parameter $\underline{q}_{i}$ represents the incremental value of $q_{i}$ which can be $\underline{u}, \underline{v}, \underline{w}, \underline{\theta}_{x}, \underline{\theta}_{y}$, or $\underline{\theta}_{z}$. The terms ${ }_{0}^{t} t_{(k)(j)}^{q_{i}}$ and ${ }_{0}^{t} t_{(j)(k)}^{q_{i}}$ represent force densities corresponding to the degree of freedom $q_{i}$. The terms $\bar{W}_{(k)}^{N L P D}$ and $\bar{W}_{(j)}^{N L P D}$ represent PD nonlinear strain energy densities of material points $k$ and $j$, respectively.

In the bond-based PD model, the force densities ${ }_{0}^{t} \mathbf{t}_{(j)(k)}$ and ${ }_{0}^{t} \mathbf{t}_{(k)(j)}$ have the same magnitude and they are in opposite directions $\left({ }_{0}^{t} \mathbf{t}_{(k)(j)}=-{ }_{0}^{t} \mathbf{t}_{(j)(k)}\right)$ [12]. Therefore, the PD equations of motion given in Eq. (24b) can be rewritten as

$\rho_{(k)}\left({ }^{t} \ddot{\mathbf{u}}_{(k)}\right)=\sum_{j=1}^{N}{ }_{0}^{t} \mathbf{f}_{(k)(j)}\left({ }^{t} \mathbf{u}_{(j)}-{ }^{t} \mathbf{u}_{(k)},{ }^{0} \mathbf{x}_{(j)}-{ }^{0} \mathbf{x}_{(k)}, t\right){ }^{0} V_{(j)}+{ }^{t} \mathbf{b}_{(k)}$

with

${ }_{0}^{t} \mathbf{f}_{(k)(j)}=2{ }_{0}^{t} \mathbf{t}_{(k)(j)}$

In the following sections, first, the nonlinear PD strain energy densities and PD bond constants for a straight beam are presented in Section 4.1. Next, the PD equations of motion in the local coordinate system of a straight beam are obtained in Section 4.2. Finally, the transformation of 
equations of motion from the beam's local coordinate system to the global coordinate system is presented in Section 4.3.

\subsection{Peridynamic strain energy density}

Similar to the classical formulations given in Eq. (23a), the nonlinear SED in PD for a beam can be represented as

$\bar{W}^{N L P D}=\bar{W}_{\text {axial }}^{N L P D}+\bar{W}_{\text {bending }}^{N L P D}+\bar{W}_{\text {shear }}^{N L P D}+\bar{W}_{\text {torsion }}^{N L P D}$

where $\bar{W}_{\text {axial }}^{N L P D}, \bar{W}_{\text {bending }}^{N L P D}, \bar{W}_{\text {shear }}^{N L P D}$, and $\bar{W}_{\text {torsion }}^{N L P D}$ represent the PD strain energy densities for axial, bending, shear, and torsional deformations, respectively. Similar to the classical formulation given in Eq. (22a), the PD strain energy density for axial deformations can be represented as

$\bar{W}_{\text {axial }}^{N L P D}=\frac{1}{2} \sum_{j=1}^{N} C_{a x}\left({ }_{0}^{t} a_{10}^{P D}\right)\left({ }_{0} \underline{a}_{10}^{P D}\right)\left({ }^{0} \xi_{(k)(j)}\right){ }^{0} V_{(j)}$

with

$$
\begin{aligned}
& { }_{0}^{t} a_{10}^{P D}={ }_{0}^{t} a_{1}^{P D}+\frac{1}{2}\left(\left({ }_{0}^{t} a_{1}^{P D}\right)^{2}+\left({ }_{0}^{t} a_{2}^{P D}\right)^{2}+\left({ }_{0}^{t} a_{3}^{P D}\right)^{2}\right) \\
& { }_{0} \underline{a}_{10}^{P D}=\left(\frac{\underline{u}_{(j)}-\underline{u}_{(k)}}{{ }^{0} \xi_{(k)(j)}}\left(1+{ }_{0}^{t} a_{1}^{P D}\right)+{ }_{0}^{t} a_{2}^{P D} \frac{\underline{\underline{v}}(j)}{{ }^{0} \underline{\xi}_{(k)(j)}}+{ }_{0}^{t} a_{3}^{P D} \frac{\underline{w}_{(j)}-\underline{w}_{(k)}}{{ }^{0} \xi_{(k)(j)}}\right)\left({ }^{0} \beta_{(k)(j)}\right) \\
& { }_{0}^{t} a_{1}^{P D}=\frac{{ }^{t} u_{(j)}-{ }^{t} u_{(k)}}{{ }^{0} \xi_{(k)(j)}} \beta_{(k)(j)} \\
& { }_{0}^{t} a_{2}^{P D}=\frac{{ }^{t} v_{(j)}-{ }^{t} v_{(k)}}{{ }^{0} \xi_{(k)(j)}}{ }^{0} \beta_{(k)(j)} \\
& { }_{0}^{t} a_{3}^{P D}=\frac{{ }^{t} w_{(j)}-{ }^{t} w_{(k)}}{{ }^{0} \xi_{(k)(j)}}{ }^{0} \beta_{(k)(j)} \\
& { }^{0} \xi_{(k)(j)}=\left|{ }^{0} x_{(j)}-{ }^{0} x_{(k)}\right| \\
& { }^{0} \beta_{(k)(j)}=\frac{{ }^{0} x_{(j)}-{ }^{0} x_{(k)}}{{ }^{0} \xi_{(k)(j)}} \\
&
\end{aligned}
$$

where $C_{a x}$ in Eq. (29a) represents PD constant for axial deformations. This PD constant can be obtained by comparing nonlinear SED for axial deformations in CCM to PD as presented in Appendix A.1 as

$$
C_{a x}=\frac{2 E}{\left({ }^{0} A\right) \delta^{2}}
$$

Similar to the classical formulation given in Eq. (22b), the nonlinear PD strain energy density for bending deformations, $\bar{W}_{\text {bending }}^{N L P D}$, in Eq. (28) can be represented as

$$
\bar{W}_{\text {bending }}^{N L P D}=\frac{C_{b z}}{2} \sum_{j=1}^{N}\left({ }_{0}^{t} a_{11}^{P D}\right)\left({ }_{0} \underline{a}_{11}^{P D}\right)\left({ }^{0} \xi_{(k)(j)}\right){ }^{0} V_{(j)}+\frac{C_{b y}}{2} \sum_{j=1}^{N}\left({ }_{0}^{t} a_{12}^{P D}\right)\left({ }_{0} \underline{a}_{12}^{P D}\right)\left({ }^{0} \xi_{(k)(j)}\right){ }^{0} V_{(j)}
$$

with

$$
{ }_{0}^{t} a_{11}^{P D}={ }_{0}^{t} a_{4}^{P D}\left(1+{ }_{0}^{t} a_{1}^{P D}\right)+\left({ }_{0}^{t} a_{2}^{P D}\right)\left({ }_{0}^{t} a_{5}^{P D}\right)+\left({ }_{0}^{t} a_{3}^{P D}\right)\left({ }_{0}^{t} a_{6}^{P D}\right)
$$




$$
\begin{aligned}
& { }_{0}^{t} a_{12}^{P D}=\left({ }_{0}^{t} a_{7}^{P D}\right)\left(1+{ }_{0}^{t} a_{1}^{P D}\right)+\left({ }_{0}^{t} a_{2}^{P D}\right)\left({ }_{0}^{t} a_{8}^{P D}\right)+\left({ }_{0}^{t} a_{3}^{P D}\right)\left({ }_{0}^{t} a_{9}^{P D}\right)
\end{aligned}
$$

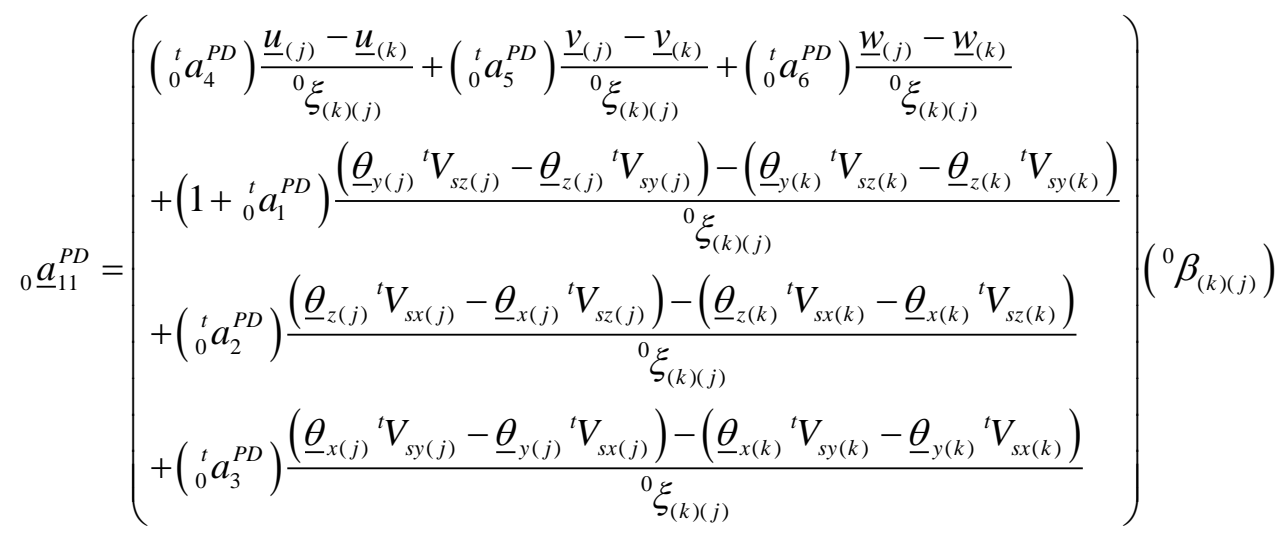

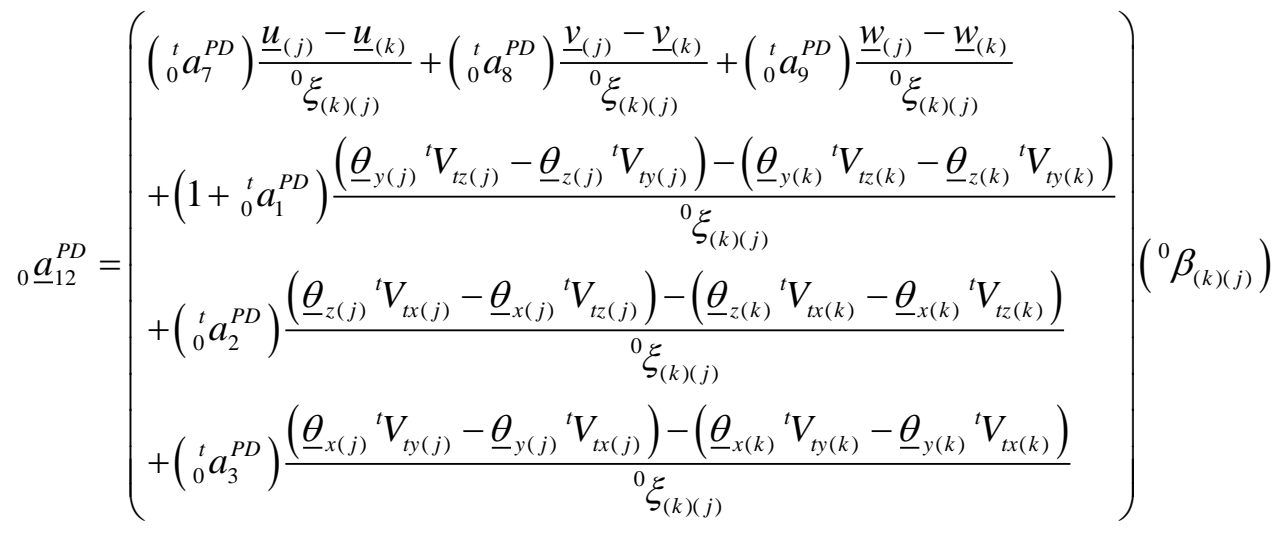

$$
\begin{aligned}
& { }_{0}^{t} a_{4}^{P D}=\frac{{ }^{t} V_{s x(j)}-{ }^{t} V_{s x(k)}}{{ }^{0} \xi_{(k)(j)}} \beta_{(k)(j)} \\
& { }_{0}^{t} a_{5}^{P D}=\frac{{ }^{t} V_{s y(j)}-{ }^{t} V_{s y(k)}}{{ }^{0} \xi_{(k)(j)}} \beta_{(k)(j)} \\
& { }_{0}^{t} a_{6}^{P D}=\frac{{ }^{t} V_{s z(j)}-{ }^{t} V_{s z(k)}}{{ }^{0} \xi_{(k)(j)}} \beta_{(k)(j)} \\
& { }_{0}^{t} a_{7}^{P D}=\frac{{ }^{t} V_{t x(j)}-{ }^{t} V_{t x(k)}}{{ }^{0} \xi_{(k)(j)}}{ }^{0} \beta_{(k)(j)} \\
& { }_{0}^{t} a_{8}^{P D}=\frac{{ }^{t} V_{t y(j)}-{ }^{t} V_{t y(k)}}{{ }^{0} \xi_{(k)(j)}} \beta_{(k)(j)}
\end{aligned}
$$


${ }_{0}^{t} a_{9}^{P D}=\frac{{ }^{t} V_{t z(j)}-{ }^{t} V_{t z(k)}}{{ }^{0} \xi_{(k)(j)}}{ }^{0} \beta_{(k)(j)}$

where the terms, $C_{b y}, C_{b z}$ in Eq. (31a) represent PD constants for bending deformations. As presented in Appendix A.2, these PD constants can be obtained by comparing nonlinear SED for bending deformations in $\mathrm{CCM}$ to $\mathrm{PD}$ as

$$
C_{b y}=\frac{2 E I_{y y}}{\left({ }^{0} A\right)^{2} \delta^{2}} ; C_{b z}=\frac{2 E I_{z z}}{\left({ }^{0} A\right)^{2} \delta^{2}}
$$

Similar to the classical formulation given in Eq. (22c), the PD strain energy density for shear deformations, $\bar{W}_{\text {shear }}^{\text {NLP }}$, in Eq. (28) can be represented as

$$
\bar{W}_{\text {shear }}^{\text {NLPD }}=\frac{1}{2} \sum_{j=1}^{N} C_{s h}\left[\left({ }_{0}^{t} a_{13}^{P D}\right)\left({ }_{0} \underline{a}_{13}^{P D}\right)+\left({ }_{0}^{t} a_{14}^{P D}\right)\left({ }_{0} \underline{a}_{14}^{P D}\right)\right]\left({ }^{0} \xi_{(k)(j)}\right)^{0} V_{(j)}
$$

with

$$
\begin{aligned}
& { }_{0}^{t} a_{13}^{P D}=\left(1+{ }_{0}^{t} a_{1}^{P D}\right) \frac{{ }^{t} V_{s x(j)}+{ }^{t} V_{s x(k)}}{2}+\left({ }_{0}^{t} a_{2}^{P D}\right) \frac{{ }^{t} V_{s y(j)}+{ }^{t} V_{s y(k)}}{2}+\left({ }_{0}^{t} a_{3}^{P D}\right) \frac{{ }^{t} V_{s z(j)}+V_{s z(k)}}{2} \\
& { }_{0}^{t} a_{14}^{P D}=\left(1+{ }_{0}^{t} a_{1}^{P D}\right) \frac{{ }^{t} V_{t x(j)}+{ }^{t} V_{t x(k)}}{2}+\left({ }_{0}^{t} a_{2}^{P D}\right) \frac{{ }^{t} V_{t y(j)}+{ }^{t} V_{t y(k)}}{2}+\left({ }_{0}^{t} a_{3}^{P D}\right) \frac{{ }^{t} V_{t z(j)}+{ }^{t} V_{t z(k)}}{2}
\end{aligned}
$$

$$
{ }_{0} \underline{a}_{13}^{P D}=\left(\begin{array}{l}
\left(\begin{array}{l}
\frac{{ }^{t} V_{s x(k)}+{ }^{t} V_{s x(j)}}{2} \frac{\underline{u}_{(j)}-\underline{u}_{(k)}}{{ }^{0} \xi_{(k)(j)}}+\frac{{ }^{t} V_{s y(k)}+{ }^{t} V_{s y(j)}}{2} \frac{\underline{v}_{(j)}-\underline{v}_{(k)}}{{ }^{0} \xi_{(k)(j)}} \\
+\frac{{ }^{t} V_{s z(k)}+{ }^{t} V_{s z(j)}}{2} \frac{\underline{w}_{(j)}-\underline{w}_{(k)}}{{ }^{0} \xi_{(k)(j)}}
\end{array}\right){ }^{0} \beta_{(k)(j)} \\
+\left(1+{ }_{0}{ }^{t} a_{1}^{P D}\right) \frac{\left(\underline{\theta}_{y(j)}{ }^{t} V_{s z(j)}-\underline{\theta}_{z(j)}{ }^{t} V_{s y(j)}\right)+\left(\underline{\theta}_{y(k)}{ }^{t} V_{s z(k)}-\underline{\theta}_{z(k)}{ }^{t} V_{s y(k)}\right)}{2} \\
+\left({ }_{0}^{t} a_{2}^{P D}\right) \frac{\left(\underline{\theta}_{z(j)}{ }^{t} V_{s x(j)}-\underline{\theta}_{x(j)}{ }^{t} V_{s z(j)}\right)+\left(\underline{\theta}_{z(k)}{ }^{t} V_{s x(k)}-\underline{\theta}_{x(k)}{ }^{t} V_{s z(k)}\right)}{2} \\
+\left({ }_{0}^{t} a_{3}^{P D}\right) \frac{\left(\underline{\theta}_{x(j)}{ }^{t} V_{s y(j)}-\underline{\theta}_{y(j)}{ }^{t} V_{s x(j)}\right)+\left(\underline{\theta}_{x(k)}{ }^{t} V_{s y(k)}-\underline{\theta}_{y(k)}{ }^{t} V_{s x(k)}\right)}{2}
\end{array}\right)
$$




$$
{ }_{0} \underline{a}_{14}^{P D}=\left(\begin{array}{l}
\left(\begin{array}{l}
\frac{{ }^{t} V_{t x(k)}+{ }^{t} V_{t x(j)}}{2} \frac{\underline{u}_{(j)}-\underline{u}_{(k)}}{{ }^{0} \xi_{(k)(j)}}+\frac{{ }^{t} V_{t y(k)}+{ }^{t} V_{t y(j)}}{2} \frac{\underline{v}_{(j)}-\underline{v}_{(k)}}{{ }^{0} \xi_{(k)(j)}} \\
+\frac{{ }^{t} V_{t z(k)}+{ }^{t} V_{t z(j)}}{2} \frac{\underline{w}_{(j)}-\underline{w}_{(k)}}{{ }^{0} \xi_{(k)(j)}}
\end{array}\right){ }^{0} \beta_{(k)(j)} \\
+\left(1+{ }_{0}^{t} a_{1}^{P D}\right) \frac{\left(\underline{\theta}_{y(j)}{ }^{t} V_{t z(j)}-\underline{\theta}_{z(j)}{ }^{t} V_{t y(j)}\right)+\left(\underline{\theta}_{y(k)}{ }^{t} V_{t z(k)}-\underline{\theta}_{z(k)}{ }^{t} V_{t y(k)}\right)}{2} \\
+\left({ }_{0}^{t} a_{2}^{P D}\right) \frac{\left(\underline{\theta}_{z(j)}{ }^{t} V_{t x(j)}-\underline{\theta}_{x(j)}{ }^{t} V_{t z(j)}\right)+\left(\underline{\theta}_{z(k)}{ }^{t} V_{t x(k)}-\underline{\theta}_{x(k)}{ }^{t} V_{t z(k)}\right)}{2} \\
+\left({ }_{0}^{t} a_{3}^{P D}\right) \frac{\left(\underline{\theta}_{x(j)}{ }^{t} V_{t y(j)}-\underline{\theta}_{y(j)}{ }^{t} V_{t x(j)}\right)+\left(\underline{\theta}_{x(k)}{ }^{t} V_{t y(k)}-\underline{\theta}_{y(k)}{ }^{t} V_{t x(k)}\right)}{2}
\end{array}\right)
$$

where the term $C_{s h}$ in Eq. (33a) represents the PD constant for shear deformations. As presented in Appendix A.3, this PD constant can be obtained by comparing nonlinear SED for shear deformations in CCM to PD as

$$
C_{s h}=\frac{2 k_{s} G}{\left({ }^{0} A\right) \delta^{2}}
$$

Similar to the classical formulation given in Eq. (22c), the PD strain energy density for torsional deformations, $\bar{W}_{\text {torsion }}^{N L P D}$, in Eq. (28) can be represented as

$$
\bar{W}_{\text {torsion }}^{N L P D}=\frac{1}{2} \sum_{j=1}^{N} C_{t}\left({ }_{0}^{t} a_{15}^{P D}\right)\left({ }_{0} \underline{a}_{15}^{P D}\right)\left({ }^{0} \xi_{(k)(j)}\right){ }^{0} V_{(j)}
$$

with

$$
\begin{aligned}
& { }_{0}^{t} a_{15}^{P D}=\left({ }_{0}^{t} a_{8}^{P D}\right) \frac{{ }^{t} V_{s y(j)}+{ }^{t} V_{s y(k)}}{2}+\left({ }_{0}^{t} a_{7}^{P D}\right) \frac{{ }^{t} V_{s x(j)}+{ }^{t} V_{s x(k)}}{2}+\left({ }_{0}^{t} a_{9}^{P D}\right) \frac{{ }^{t} V_{s z(j)}+{ }^{t} V_{s z(k)}}{2} \\
& 0 \underline{a}_{15}^{P D}=\left(\begin{array}{l}
\left(\frac{{ }^{t} V_{t y(j)}+{ }^{t} V_{t y(k)}}{2} \frac{{ }^{t} V_{s z(j)}+{ }^{t} V_{s z(k)}}{2}-\frac{{ }^{t} V_{t z(j)}+{ }^{t} V_{t z(k)}}{2} \frac{{ }^{t} V_{s y(k)}+{ }^{t} V_{s y(j)}}{2}\right) \frac{\underline{\theta}_{x(j)}-\underline{\theta}_{x(k)}}{{ }^{0} \xi_{(k)(j)}} \\
+\left(\frac{{ }^{t} V_{t z(j)}+{ }^{t} V_{t z(k)}}{2} \frac{{ }^{t} V_{s x(j)}+{ }^{t} V_{s x(k)}}{2}-\frac{{ }^{t} V_{t x(j)}+{ }^{t} V_{t x(k)}}{2} \frac{{ }^{t} V_{s z(j)}+{ }^{t} V_{s z(k)}}{2}\right) \frac{\underline{\theta}_{y(j)}-\underline{\theta}_{y(k)}}{{ }^{0} \xi_{(k)(j)}} \\
+\left(\frac{{ }^{t} V_{t x(j)}+{ }^{t} V_{t x(k)}}{2} \frac{{ }^{t} V_{s y(k)}+{ }^{t} V_{s y(j)}}{2}-\frac{{ }^{t} V_{t y(j)}+{ }^{t} V_{t y(k)}}{2} \frac{{ }^{t} V_{s x(j)}+{ }^{t} V_{s x(k)}}{2}\right) \frac{\underline{\theta}_{z(j)(j)}-\underline{\theta}_{z(k)}}{{ }^{0} \xi_{(k)(j)}}
\end{array}\right)
\end{aligned}
$$

where the term $C_{t}$ in Eq. (35a) represents the PD constant for torsional deformations. As presented in Appendix A.4, this PD constant can be obtained by comparing nonlinear SED for torsional deformations in CCM to PD as

$$
C_{t}=\frac{2 k_{t} G}{\left({ }^{0} A\right)^{2} \delta^{2}}
$$

Note that the PD constants given in Eq. (30), Eq. (32), Eq. (34), and Eq. (36) in this study have the same forms as the PD constants for linear beam given in [66]. However, as provided in Appendix 
A, in this study, the PD constants for nonlinear beams were obtained by comparing nonlinear SED components in PD to those in classical continuum mechanics. In [66], the PD constants for linear beams were obtained by comparing linear SED components in PD to those in classical continuum mechanics.

\subsection{Nonlinear equation of motion in the local coordinate system for a beam}

By substituting the nonlinear SED given in Eq. (28) into Eq. (26a), the PD force densities ${ }_{0}^{t} t_{(k)(j)}^{q_{i}}$ can be represented as

$$
\begin{aligned}
& { }_{0}^{t} t_{(k)(j)}^{u}=\frac{1}{2}\left\{\begin{array}{l}
C_{a x}\left({ }_{0}^{t} a_{10}^{P D}\right)\left(1+{ }_{0}^{t} a_{1}^{P D}\right)+C_{b z}\left({ }_{0}^{t} a_{11}^{P D}\right)\left({ }_{0}^{t} a_{4}^{P D}\right)+C_{b y}\left({ }_{0}^{t} a_{12}^{P D}\right)\left({ }_{0}^{t} a_{7}^{P D}\right) \\
+C_{s h}\left(\left({ }_{0}^{t} a_{13}^{P D}\right) \frac{{ }^{t} V_{s x(k)}+{ }^{t} V_{s x(i)}}{2}+\left({ }_{0}^{t} a_{14}^{P D}\right) \frac{{ }^{t} V_{t x(k)}+{ }^{t} V_{t x(i)}}{2}\right)
\end{array}\right\}\left({ }^{0} \xi_{(k)(j)}\right) \\
& { }_{0}^{t} t_{(k)(j)}^{v}=\frac{1}{2}\left\{\begin{array}{l}
C_{a x}\left({ }_{0}^{t} a_{10}^{P D}\right)\left({ }_{0}^{t} a_{2}^{P D}\right)+C_{b z}\left({ }_{0}^{t} a_{11}^{P D}\right)\left({ }_{0}^{t} a_{5}^{P D}\right)+C_{b y}\left({ }_{0}^{t} a_{12}^{P D}\right)\left({ }_{0}^{t} a_{8}^{P D}\right) \\
+C_{s h}\left(\left({ }_{0}^{t} a_{13}^{P D}\right) \frac{{ }^{t} V_{s y(k)}+{ }^{t} V_{s y(j)}}{2}+\left({ }_{0}^{t} a_{14}^{P D}\right) \frac{{ }^{t} V_{t y(k)}+{ }^{t} V_{t y(j)}}{2}\right)
\end{array}\right\}\left({ }^{0} \xi_{(k)(j)}\right) \\
& { }_{0}^{t} t_{(k)(j)}^{w}=\frac{1}{2}\left\{\begin{array}{l}
C_{a x}\left({ }_{0}^{t} a_{10}^{P D}\right)\left({ }_{0}^{t} a_{3}^{P D}\right)+C_{b z}\left({ }_{0}^{t} a_{11}^{P D}\right)\left({ }_{0}^{t} a_{6}^{P D}\right)+C_{b y}\left({ }_{0}^{t} a_{12}^{P D}\right)\left({ }_{0}^{t} a_{9}^{P D}\right) \\
C_{s h}\left(\left({ }_{0}^{t} a_{13}^{P D}\right) \frac{{ }^{t} V_{s z(k)}+{ }^{t} V_{s z(j)}}{2}+\left({ }_{0}^{t} a_{14}^{P D}\right) \frac{{ }^{t} V_{t z(k)}+{ }^{t} V_{t z(j)}}{2}\right)
\end{array}\right\}\left({ }^{0} \xi_{(k)(j)}\right) \\
& { }_{0}^{t} t_{(k)(j)}^{\theta_{x}}=\frac{1}{2} C_{b z}\left({ }_{0}^{t} a_{11}^{P D}\right)\left\{-\left({ }_{0}^{t} a_{2}^{P D}\right){ }^{t} V_{s z(k)}+\left({ }_{0}^{t} a_{3}^{P D}\right){ }^{t} V_{s y(k)}\right\}\left({ }^{0} \beta_{(k)(j)}\right) \\
& +\frac{1}{2} C_{b y}\left({ }_{0}^{t} a_{12}^{P D}\right)\left\{-\left({ }_{0}^{t} a_{2}^{P D}\right){ }^{t} V_{t z(k)}+\left({ }_{0}^{t} a_{3}^{P D}\right){ }^{t} V_{t y(k)}\right\}\left({ }^{0} \beta_{(k)(j)}\right) \\
& +\frac{1}{4} C_{s h}\left({ }^{0} \xi_{(k)(j)}\right)\left[\begin{array}{l}
\left({ }_{0}^{t} a_{13}^{P D}\right)\left\{\left({ }_{0}^{t} a_{2}^{P D}\right)^{t} V_{s z(k)}-\left({ }_{0}^{t} a_{3}^{P D}\right)^{t} V_{s y(k)}\right\} \\
+\left({ }_{0}^{t} a_{14}^{P D}\right)\left\{\left({ }_{0}^{t} a_{2}^{P D}\right){ }^{t} V_{t z(k)}-\left({ }_{0}^{t} a_{3}^{P D}\right){ }^{t} V_{t y(k)}\right\}
\end{array}\right] \\
& +\frac{1}{2} C_{t}\left({ }_{0}^{t} a_{15}^{P D}\right)\left(\begin{array}{l}
\frac{{ }^{t} V_{t y(j)}+{ }^{t} V_{t y(k)}}{2} \frac{{ }^{t} V_{s z(j)}+{ }^{t} V_{s z(k)}}{2} \\
-\frac{{ }^{t} V_{t z(j)}+{ }^{t} V_{t z(k)}}{2} \frac{{ }^{t} V_{s y(k)}+{ }^{t} V_{s y(j)}}{2}
\end{array}\right)\left({ }^{0} \beta_{(k)(j)}\right)
\end{aligned}
$$




$$
\begin{aligned}
& { }_{0}^{t} t_{(k)(j)}^{\theta_{y}}=\frac{1}{2} C_{b z}\left({ }_{0}^{t} a_{11}^{P D}\right)\left\{\left(1+{ }_{0}^{t} a_{1}^{P D}\right){ }^{t} V_{s z(k)}-\left({ }_{0}^{t} a_{3}^{P D}\right){ }^{t} V_{s x(k)}\right\}\left({ }^{0} \beta_{(k)(j)}\right) \\
& +\frac{1}{2} C_{b y}\left({ }_{0}^{t} a_{12}^{P D}\right)\left\{\left(1+{ }_{0}^{t} a_{1}^{P D}\right){ }^{t} V_{t z(k)}-\left({ }_{0}^{t} a_{3}^{P D}\right){ }^{t} V_{t x(k)}\right\}\left({ }^{0} \beta_{(k)(j)}\right) \\
& -\frac{1}{4} C_{s h}\left({ }^{0} \xi_{(k)(j)}\right)\left[\begin{array}{l}
\left({ }_{0}^{t} a_{13}^{P D}\right)\left\{\left(1+{ }_{0}^{t} a_{1}^{P D}\right){ }^{t} V_{s z(k)}-\left({ }_{0}^{t} a_{3}^{P D}\right)^{t} V_{s x(k)}\right\} \\
+\left({ }_{0}^{t} a_{14}^{P D}\right)\left\{\left(1+{ }_{0}^{t} a_{1}^{P D}\right){ }^{t} V_{t z(k)}-\left({ }_{0}^{t} a_{3}^{P D}\right){ }^{t} V_{t x(k)}\right\}
\end{array}\right] \\
& +\frac{1}{2} C_{t}\left({ }_{0}^{t} a_{15}^{P D}\right)\left(\begin{array}{l}
\frac{{ }^{t} V_{t z(j)}+{ }^{t} V_{t z(k)}}{2} \frac{{ }^{t} V_{s x(j)}+{ }^{t} V_{s x(k)}}{2} \\
-\frac{{ }^{t} V_{t x(j)}+{ }^{t} V_{t x(k)}}{2} \frac{{ }^{t} V_{s z(j)}+{ }^{t} V_{s z(k)}}{2}
\end{array}\right)\left({ }^{0} \beta_{(k)(j)}\right) \\
& { }_{0}^{t} t_{(k)(j)}^{\theta_{z}}=\frac{1}{2} C_{b z}\left({ }_{0}^{t} a_{11}^{P D}\right)\left\{-\left(1+{ }_{0}^{t} a_{1}^{P D}\right){ }^{t} V_{s y(k)}+\left({ }_{0}^{t} a_{2}^{P D}\right){ }^{t} V_{s x(k)}\right\}\left({ }^{0} \beta_{(k)(j)}\right) \\
& +\frac{1}{2} C_{b y}\left({ }_{0}^{t} a_{12}^{P D}\right)\left\{-\left(1+{ }_{0}^{t} a_{1}^{P D}\right){ }^{t} V_{t y(k)}+\left({ }_{0}^{t} a_{2}^{P D}\right){ }^{t} V_{t x(k)}\right\}\left({ }^{0} \beta_{(k)(j)}\right) \\
& +\frac{1}{4} C_{s h}\left({ }^{0} \xi_{(k)(j)}\right)\left[\begin{array}{l}
\left({ }_{0}^{t} a_{13}^{P D}\right)\left\{\left(1+{ }_{0}^{t} a_{1}^{P D}\right){ }^{t} V_{s y(k)}-\left({ }_{0}^{t} a_{2}^{P D}\right)^{t} V_{s x(k)}\right\} \\
+\left({ }_{0}^{t} a_{14}^{P D}\right)\left\{\left(1+{ }_{0}^{t} a_{1}^{P D}\right){ }^{t} V_{t y(k)}-\left({ }_{0}^{t} a_{2}^{P D}\right){ }^{t} V_{t x(k)}\right\}
\end{array}\right] \\
& +\frac{1}{2} C_{t}\left({ }_{0}^{t} a_{15}^{P D}\right)\left(\begin{array}{l}
\frac{{ }_{t}^{t} V_{t x(j)}+{ }^{t} V_{t x(k)}}{2} \frac{{ }^{t} V_{s y(k)}+{ }^{t} V_{s y(j)}}{2} \\
-\frac{{ }^{t} V_{t y(j)}+{ }^{t} V_{t y(k)}}{2} \frac{{ }^{t} V_{s x(j)}+{ }^{t} V_{s x(k)}}{2}
\end{array}\right)\left({ }^{0} \beta_{(k)(j)}\right)
\end{aligned}
$$

Therefore, by substituting PD force densities given in Eq. (37) into Eq. (27), the nonlinear PD equations of motion for a straight beam can be obtained as

$$
\begin{aligned}
& \rho\left({ }^{t} \ddot{u}_{(k)}\right)=\sum_{j=1}^{N}\left({ }_{0}^{t} f_{(k)(j)}^{u}\right){ }^{0} V_{(j)}+{ }^{t} b_{x(k)} \\
& \rho\left({ }^{t} \ddot{v}_{(k)}\right)=\sum_{j=1}^{N}\left({ }_{0}^{t} f_{(k)(j)}^{v}\right){ }^{0} V_{(j)}+{ }^{t} b_{y(k)} \\
& \rho\left({ }^{t} \ddot{w}_{(k)}\right)=\sum_{j=1}^{N}\left({ }_{0}^{t} f_{(k)(j)}^{w}\right){ }^{0} V_{(j)}+{ }^{t} b_{w(k)} \\
& \frac{\rho I_{x x}}{A}\left({ }^{t} \ddot{\theta}_{x(k)}\right)=\sum_{j=1}^{N}\left({ }_{0}^{t} f_{(k)(j)}^{\theta_{x}}\right){ }^{0} V_{(j)}+{ }^{t} m_{x(k)} \\
& \frac{\rho I_{y y}}{A}\left({ }^{t} \ddot{\theta}_{y(k)}\right)=\sum_{j=1}^{N}\left({ }_{0}^{t} f_{(k)(j)}^{\theta_{y}}\right){ }^{0} V_{(j)}+{ }^{t} m_{y(k)} \\
& \frac{\rho I_{z z}}{A}\left({ }^{t} \ddot{\theta}_{z(k)}\right)=\sum_{j=1}^{N}\left({ }_{0}^{t} f_{(k)(j)}^{\theta_{z}}\right){ }^{0} V_{(j)}+{ }^{t} m_{z(k)}
\end{aligned}
$$

with

${ }_{0}^{t} f_{(k)(j)}^{u}=2\left({ }_{0}^{t} t_{(k)(j)}^{u}\right) ; \quad{ }_{0}^{t} f_{(k)(j)}^{v}=2\left({ }_{0}^{t} t_{(k)(j)}^{v}\right) ; \quad{ }_{0}^{t} f_{(k)(j)}^{w}=2\left({ }_{0}^{t} t_{(k)(j)}^{w}\right)$ 
${ }_{0}^{t} f_{(k)(j)}^{\theta_{x}}=2\left({ }_{0}^{t} t_{(k)(j)}^{\theta_{x}}\right) ; \quad{ }_{0}^{t} f_{(k)(j)}^{\theta_{y}}=2\left({ }_{0}^{t} t_{(k)(j)}^{\theta_{y}}\right) ; \quad{ }_{0}^{t} f_{(k)(j)}^{\theta_{z}}=2\left({ }_{0}^{t} t_{(k)(j)}^{\theta_{z}}\right)$

The equations of motion given in Eq. (38) can be rewritten in matrix form as $[35,66]$

$\mathbf{m}_{(k)}^{L}\left({ }^{t} \ddot{\mathbf{u}}_{(k)}^{L}\right)=\sum_{j=1}^{N}\left({ }_{0}^{t} \mathbf{f}_{(k)(j)}^{L}\right){ }^{0} V_{(j)}+{ }^{t} \mathbf{b}_{(k)}^{L}$

where $\mathbf{m}_{(k)}^{L},{ }^{t} \mathbf{b}_{(k)}^{L},{ }_{0}^{t} \mathbf{f}_{(k)(j)}^{L}$ and ${ }^{t} \ddot{\mathbf{u}}_{(k)}^{L}$ represent mass matrix, body force vector, PD force density vector, and acceleration vector in the local coordinate system, respectively. These terms can be defined as

$$
\begin{aligned}
& \mathbf{m}_{(k)}^{L}=\left[\begin{array}{cccccc}
\rho & 0 & 0 & 0 & 0 & 0 \\
0 & \rho & 0 & 0 & 0 & 0 \\
0 & 0 & \rho & 0 & 0 & 0 \\
0 & 0 & 0 & \frac{\rho I_{x x}}{A} & 0 & 0 \\
0 & 0 & 0 & 0 & \frac{\rho I_{y y}}{A} & 0 \\
0 & 0 & 0 & 0 & 0 & \frac{\rho I_{z z}}{A}
\end{array}\right] ;{ }^{t} \ddot{\mathbf{u}}_{(k)}^{L}=\left[\begin{array}{c}
{ }^{t} \ddot{u}_{(k)} \\
{ }^{t} \ddot{v}_{(k)} \\
{ }^{t} \ddot{w}_{(k)} \\
{ }^{t} \ddot{\theta}_{x(k)} \\
{ }^{t} \ddot{\theta}_{y(k)} \\
{ }^{t} \ddot{\theta}_{z(k)}
\end{array}\right] ;{ }^{t} \mathbf{b}_{(k)}^{L}=\left[\begin{array}{c}
{ }^{t} b_{x(k)} \\
{ }^{t} b_{y(k)} \\
{ }^{t} b_{z(k)} \\
{ }^{t} m_{x(k)} \\
{ }^{t} m_{y(k)} \\
{ }^{t} m_{z(k)}
\end{array}\right]
\end{aligned}
$$

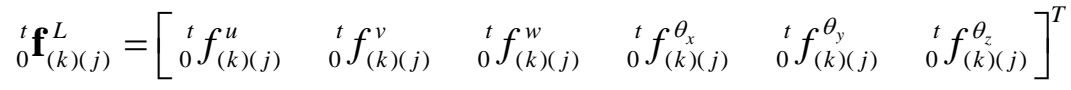

\subsection{Transformation of the equation of motion to the global coordinate system}

As given in Eq. (39), the PD equations of motion for a beam are derived with respect to the beam's local coordinate system. Therefore, to investigate complex beam structures and curved beams, the equations of motion in the local coordinate system need to be transformed to the global coordinate system [66]. The global coordinate system is fixed and it is often chosen as the Cartesian coordinate system $\left(x_{1}, x_{2}, x_{3}\right)$ as shown in Fig. 4. The beam has six global degrees of freedom denoted as $\left(u_{1}, u_{2}, u_{3}, \theta_{1}, \theta_{2}, \theta_{3}\right)$. 


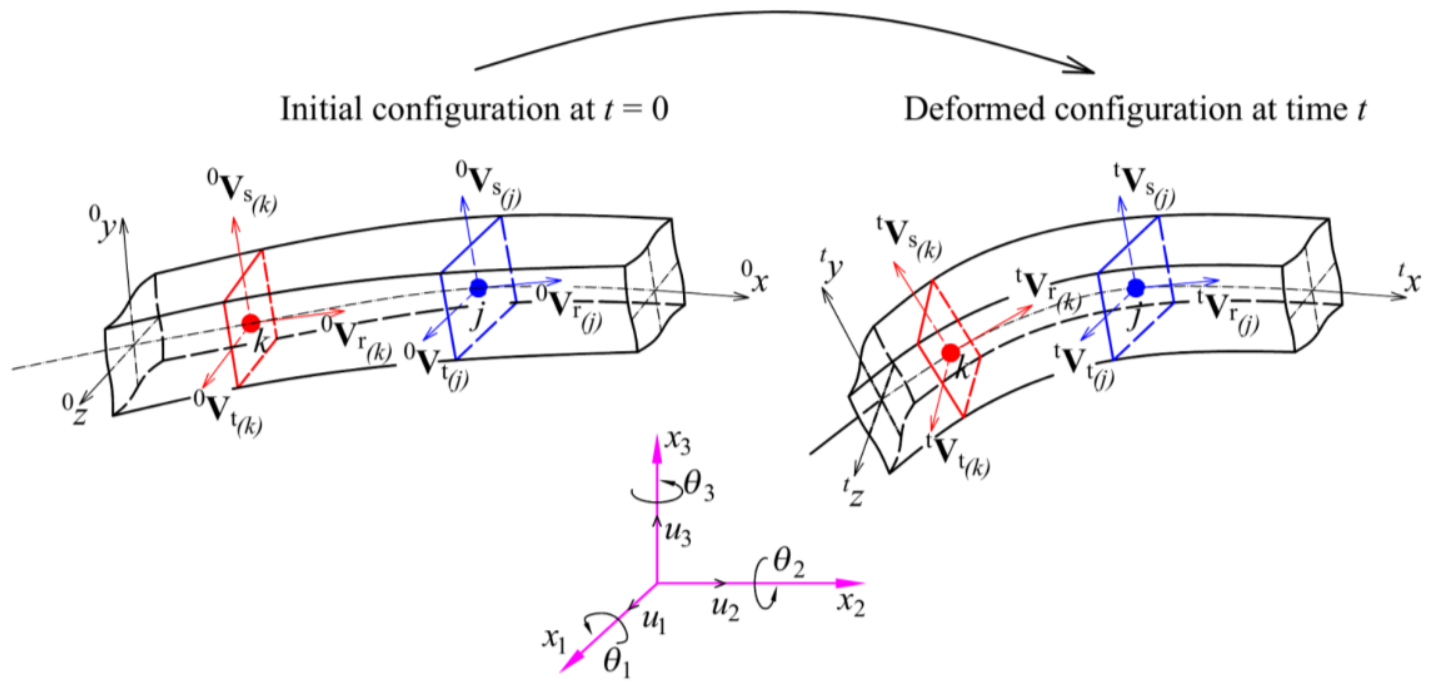

Fig. 4. Beam configurations in the global coordinate system

As presented in section 3 , each material point has three director vectors $\left({ }^{0} \mathbf{V}_{r},{ }^{0} \mathbf{V}_{s},{ }^{0} \mathbf{V}_{t}\right)$ or $\left({ }^{t} \mathbf{V}_{r},{ }^{t} \mathbf{V}_{s},{ }^{t} \mathbf{V}_{t}\right)$ measured in the local coordinate system. These director vectors can be transformed to the global coordinate system by using the following relations as

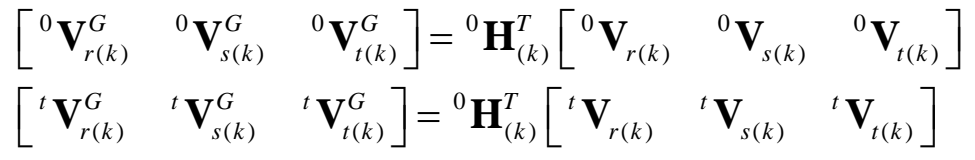

with

${ }^{0} \mathbf{H}_{(k)}=\left[\begin{array}{ccc}{ }^{0} \mathbf{V}_{r(k)}^{G} & { }^{0} \mathbf{V}_{s(k)}^{G} & { }^{0} \mathbf{V}_{t(k)}^{G}\end{array}\right]^{T}$

where ${ }^{0} \mathbf{H}_{(k)}$ represents the coordinate transformation matrix for material point $k[35,66],{ }^{0} \mathbf{H}_{(k)}^{T}$ is transpose matrix of ${ }^{0} \mathbf{H}_{(k)}$. The terms $\left({ }^{0} \mathbf{V}_{r(k)},{ }^{0} \mathbf{V}_{s(k)},{ }^{0} \mathbf{V}_{t(k)}\right)$ and $\left({ }^{t} \mathbf{V}_{r(k)},{ }^{t} \mathbf{V}_{s(k)},{ }^{t} \mathbf{V}_{t(k)}\right)$ are the director vectors in local coordinates at $t=0$ and at time $t$, respectively. Meanwhile, $\left({ }^{0} \mathbf{V}_{r(k)}^{G}, \quad{ }^{0} \mathbf{V}_{s(k)}^{G}, \quad{ }^{0} \mathbf{V}_{t(k)}^{G}\right)$ and $\left({ }^{t} \mathbf{V}_{r(k)}^{G}, \quad{ }^{t} \mathbf{V}_{s(k)}^{G}, \quad{ }^{t} \mathbf{V}_{t(k)}^{G}\right)$ are the director vectors in the global coordinates at $t=0$ and at time $t$, respectively. As given in Eq. (11), the director vectors in local coordinates at $t=0$ are presented as

$$
\begin{aligned}
{ }^{0} \mathbf{V}_{r(k)} & =\left[\begin{array}{lll}
1 & 0 & 0
\end{array}\right]^{T} \\
{ }^{0} \mathbf{V}_{s(k)} & =\left[\begin{array}{lll}
0 & 1 & 0
\end{array}\right]^{T} \\
{ }^{0} \mathbf{V}_{t(k)} & =\left[\begin{array}{lll}
0 & 0 & 1
\end{array}\right]^{T}
\end{aligned}
$$

The global director vectors at time $t$ given in Eq. (41b) can be estimated based on the director vectors in the previous configuration as [7]

$$
\begin{aligned}
& { }^{t} \overline{\mathbf{V}}_{s(k)}^{G}={ }^{t-\Delta t} \mathbf{V}_{s(k)}^{G}+\underline{\boldsymbol{\theta}}_{(k)}^{G} \times{ }^{t-\Delta t} \mathbf{V}_{s(k)}^{G} \\
& { }^{t} \overline{\mathbf{V}}_{t(k)}^{G}={ }^{t-\Delta t} \mathbf{V}_{t(k)}^{G}+\underline{\boldsymbol{\theta}}_{(k)}^{G} \times{ }^{t-\Delta t} \mathbf{V}_{t(k)}^{G}
\end{aligned}
$$

and 
${ }^{t} \mathbf{V}_{s(k)}=\frac{{ }^{t} \overline{\mathbf{V}}_{s(k)}^{G}}{\left|{ }^{t} \overline{\mathbf{V}}_{s(k)}^{G}\right|}$

${ }^{t} \mathbf{V}_{t(k)}^{G}=\frac{{ }^{t} \overline{\mathbf{V}}_{t(k)}^{G}}{\left|{ }^{t} \overline{\mathbf{V}}_{t(k)}^{G}\right|}$

${ }^{t} \mathbf{V}_{r(k)}^{G}={ }^{t} \mathbf{V}_{s(k)}^{G} \times{ }^{t} \mathbf{V}_{t(k)}^{G}$

where ${ }^{t} \overline{\mathbf{V}}_{s(k)}^{G}$ and ${ }^{t} \overline{\mathbf{V}}_{t(k)}^{G}$ have the same directions with director vectors, ${ }^{t} \mathbf{V}_{s(k)}^{G}$ and ${ }^{t} \mathbf{V}_{t(k)}^{G}$. However, ${ }^{t} \overline{\mathbf{V}}_{s(k)}^{G}$ and ${ }^{t} \overline{\mathbf{V}}_{t(k)}^{G}$ may not be unit vectors. Therefore, to ensure the director vectors are unit vectors, the approximations in Eq. (43c-d) are used. The term $\underline{\boldsymbol{\theta}}_{(k)}^{G}$ represents the vector of the global incremental rotations from time $t-\Delta t$ to time $t$. This vector can be defined as

$\underline{\boldsymbol{\theta}}_{(k)}^{G}={ }^{t} \underline{\boldsymbol{\theta}}_{(k)}^{G}-{ }^{t-\Delta t} \underline{\boldsymbol{\theta}}_{(k)}^{G}=\left[\begin{array}{lll}\underline{\theta}_{1(k)} & \underline{\theta}_{2(k)} & \underline{\theta}_{3(k)}\end{array}\right]^{T}$

As given in Eq. (41c), ${ }^{0} \mathbf{H}_{(k)}$ is the coordinate transformation matrix for material point $k$. Therefore, for the interaction between material points $k$ and $j$, the coordinate transformation matrix can be approximated as $[35,66]$

${ }^{0} \mathbf{H}_{(k)(j)}=\left[\begin{array}{lll}{ }^{0} \mathbf{V}_{r(k)(j)}^{G} & { }^{0} \mathbf{V}_{s(k)(j)}^{G} & { }^{0} \mathbf{V}_{t(k)(j)}^{G}\end{array}\right]^{T}$

with

${ }^{0} \mathbf{V}_{r(k)(j)}^{G}=\frac{\left({ }^{0} \mathbf{V}_{r(k)}^{G}+{ }^{0} \mathbf{V}_{r(j)}^{G}\right)}{\left|\left({ }^{0} \mathbf{V}_{r(k)}^{G}+{ }^{0} \mathbf{V}_{r(j)}^{G}\right)\right|}$

${ }^{0} \mathbf{V}_{s(k)(j)}^{G}=\frac{\left({ }^{0} \mathbf{V}_{s(k)}^{G}+{ }^{0} \mathbf{V}_{s(j)}^{G}\right)}{\left|\left({ }^{0} \mathbf{V}_{s(k)}^{G}+{ }^{0} \mathbf{V}_{s(j)}^{G}\right)\right|}$

${ }^{0} \mathbf{V}_{t(k)(j)}^{G}=\frac{\left({ }^{0} \mathbf{V}_{t(k)}^{G}+{ }^{0} \mathbf{V}_{t(j)}^{G}\right)}{\left|\left({ }^{0} \mathbf{V}_{t(k)}^{G}+{ }^{0} \mathbf{V}_{t(j)}^{G}\right)\right|}$

Therefore, the transformation matrix for six degrees of freedom of the beam, ${ }^{0} \mathbf{T}_{(k)(j)}$, can be defined as

${ }^{0} \mathbf{T}_{(k)(j)}=\left[\begin{array}{cc}{ }^{0} \mathbf{H}_{(k)(j)} & \mathbf{0} \\ \mathbf{0} & { }^{0} \mathbf{H}_{(k)(j)}\end{array}\right]$

By using the transformation matrix ${ }^{0} \mathbf{T}_{(k)(j)}$, the relationship between the local and global degrees of freedom can be presented as [35, 66]

$$
\begin{aligned}
{ }^{t} \mathbf{u}_{(k)}^{L} & ={ }^{0} \mathbf{T}_{(k)(j)}{ }^{t} \mathbf{u}_{(k)}^{G} \\
{ }^{t} \mathbf{u}_{(j)}^{L} & ={ }^{0} \mathbf{T}_{(k)(j)}{ }^{t} \mathbf{u}_{(j)}^{G}
\end{aligned}
$$

with

$$
\begin{aligned}
{ }^{t} \mathbf{u}_{(k)}^{L} & =\left[\begin{array}{llllll}
{ }^{t} u_{(k)} & { }^{t} v_{(k)} & { }^{t} w_{(k)} & { }^{t} \theta_{x(k)} & { }^{t} \theta_{y(k)} & { }^{t} \theta_{z(k)}
\end{array}\right] \\
{ }^{t} \mathbf{u}_{(k)}^{G} & =\left[\begin{array}{llllll}
{ }^{t} u_{1(k)} & { }^{t} u_{2(k)} & { }^{t} u_{3(k)} & { }^{t} \theta_{1(k)} & { }^{t} \theta_{2(k)} & { }^{t} \theta_{3(k)}
\end{array}\right]
\end{aligned}
$$


${ }^{t} \mathbf{u}_{(j)}^{L}=\left[\begin{array}{llllll}{ }^{t} u_{(j)} & { }^{t} v_{(j)} & { }^{t} w_{(j)} & { }^{t} \theta_{x(j)} & { }^{t} \theta_{y(j)} & { }^{t} \theta_{z(j)}\end{array}\right]^{T}$

${ }^{t} \mathbf{u}_{(j)}^{G}=\left[\begin{array}{llllll}{ }^{t} u_{1(j)} & { }^{t} u_{2(j)} & { }^{t} u_{3(j)} & { }^{t} \theta_{1(j)} & { }^{t} \theta_{2(j)} & { }^{t} \theta_{3(j)}\end{array}\right]^{T}$

where ${ }^{t} \mathbf{u}_{(k)}^{L}$ and ${ }^{t} \mathbf{u}_{(j)}^{L}$ represent vectors of DOFs in local coordinates for material points $k$ and $j$ , respectively. Meanwhile, ${ }^{t} \mathbf{u}_{(k)}^{G}$ and ${ }^{t} \mathbf{u}_{(j)}^{G}$ represent vectors of DOFs in global coordinates for material points $k$ and $j$, respectively.

By multiplying both sides of Eq. (39) with the matrix ${ }^{0} \mathbf{T}_{(k)(j)}^{T}$, the nonlinear equation of motion for a beam can be transformed to the global coordinate system as $[35,66]$

${ }^{0} \mathbf{T}_{(k)(j)}^{T} \mathbf{m}_{(k)}^{L}\left({ }^{0} \mathbf{T}_{(k)(j)}{ }^{t} \ddot{\mathbf{u}}_{(k)}^{G}\right)=\sum_{j=1}^{N}{ }^{0} \mathbf{T}_{(k)(j)}^{T}\left({ }_{0}^{t} \mathbf{f}_{(k)(j)}^{L}{ }^{0} V_{(j)}+{ }^{0} \mathbf{T}_{(k)(j)}^{T}{ }^{t} \mathbf{b}_{(k)}^{L}\right.$

or

$\mathbf{m}_{(k)}^{G}\left({ }^{t} \ddot{\mathbf{u}}_{(k)}^{G}\right)=\sum_{j=1}^{N}\left({ }_{0}^{t} \mathbf{f}_{(k)(j)}^{G}\right){ }^{0} V_{(j)}+{ }^{t} \mathbf{b}_{(k)}^{G}$

with

$$
\begin{aligned}
& \mathbf{m}_{(k)}^{G}={ }^{0} \mathbf{T}_{(k)(j)}^{T} \mathbf{m}_{(k)}^{L}{ }^{0} \mathbf{T}_{(k)(j)} \\
& { }_{0}^{t} \mathbf{f}_{(k)(j)}^{G}={ }^{0} \mathbf{T}_{(k)(j)}^{T}\left({ }_{0}^{t} \mathbf{f}_{(k)(j)}^{L}\right)=\left[\begin{array}{lllllll}
{ }_{0}^{t} f_{(k)(j)}^{u_{1}} & { }_{0}^{t} f_{(k)(j)}^{u_{2}} & { }_{0}^{t} f_{(k)(j)}^{u_{3}} & { }_{0}^{t} f_{(k)(j)}^{\theta_{1}} & { }_{0}^{t} f_{(k)(j)}^{\theta_{2}} & { }_{0}^{t} f_{(k)(j)}^{\theta_{3}}
\end{array}\right]^{T} \\
& { }^{t} \mathbf{b}_{(k)}^{G}={ }^{G} \mathbf{T}_{(k)(j)}^{T}\left({ }^{t} \mathbf{b}_{(k)}^{L}\right)=\left[\begin{array}{llllll}
{ }^{t} b_{1(k)} & { }^{t} b_{2(k)} & { }^{t} b_{3(k)} & { }^{t} m_{1(k)} & { }^{t} m_{2(k)} & { }^{t} m_{3(k)}
\end{array}\right]^{T}
\end{aligned}
$$

where ${ }^{0} \mathbf{T}_{(k)(j)}^{T}$ is the transpose matrix of ${ }^{0} \mathbf{T}_{(k)(j)}$. The terms $\mathbf{m}_{(k)}^{G},{ }^{t} \ddot{\mathbf{u}}_{(k)}^{G},{ }^{t} \mathbf{f}_{(k)(j)}^{G}$ and ${ }^{t} \mathbf{b}_{(k)}^{G}$ represent mass matrix, acceleration vector, PD force density vector, and the applied body force vector in the global coordinate system, respectively.

Note that term ${ }^{0} \beta_{(k)(j)}$ given in Eq. (29h) can be calculated in global coordinates as

$$
{ }^{0} \beta_{(k)(j)}=\frac{{ }^{0} \mathbf{V}_{r(k)(j)}^{G} \cdot\left({ }^{0} \mathbf{x}_{(j)}^{G}-{ }^{0} \mathbf{x}_{(k)}^{G}\right)}{\left|{ }^{0} \mathbf{V}_{r(k)(j)}^{G} \cdot\left({ }^{0} \mathbf{x}_{(j)}^{G}-{ }^{0} \mathbf{x}_{(k)}^{G}\right)\right|}=\left\{\begin{array}{lll}
-1 & \text { if } & { }^{0} \mathbf{V}_{r(k)(j)}^{G} \uparrow \downarrow\left({ }^{0} \mathbf{x}_{(j)}^{G}-{ }^{0} \mathbf{x}_{(k)}^{G}\right) \\
1 & \text { if } & { }^{0} \mathbf{V}_{r(k)(j)}^{G} \uparrow \uparrow\left({ }^{0} \mathbf{x}_{(j)}^{G}-{ }^{0} \mathbf{x}_{(k)}^{G}\right)
\end{array}\right.
$$

with

$$
\begin{aligned}
& { }^{0} \mathbf{x}_{(k)}^{G}=\left[\begin{array}{lll}
{ }^{0} x_{1(k)} & { }^{0} x_{2(k)} & { }^{0} x_{3(k)}
\end{array}\right]^{T} \\
& { }^{0} \mathbf{x}_{(j)}^{G}=\left[\begin{array}{lll}
{ }^{0} x_{1(j)} & { }^{0} x_{2(j)} & { }^{0} x_{3(j)}
\end{array}\right]^{T}
\end{aligned}
$$

where ${ }^{0} \mathbf{x}_{(k)}^{G}$ and ${ }^{0} \mathbf{x}_{(j)}^{G}$ represent the global coordinates in the initial configuration of material points $k$ and $j$, respectively. Note that, the parameter ${ }^{0} \beta_{(k)(j)}$ in Eq. (49a) equal to -1 if two vectors ${ }^{0} \mathbf{V}_{r(k)(j)}^{G}$ and $\left({ }^{0} \mathbf{x}_{(j)}^{G}-{ }^{0} \mathbf{x}_{(k)}^{G}\right)$ have opposite directions. On the other hand, ${ }^{0} \beta_{(k)(j)}$ equal to 1 if two vectors ${ }^{0} \mathbf{V}_{r(k)(j)}^{G}$ and $\left({ }^{0} \mathbf{x}_{(j)}^{G}-{ }^{0} \mathbf{x}_{(k)}^{G}\right)$ have the same directions. 


\section{Damage prediction}

Progressive damages are introduced in peridynamics by eliminating interactions between material points. The state of interaction between material points $k$ and $j$, can be represented by a damage parameter, $\psi_{(k)(j)}$, as [12]

$\psi_{(k)(j)}\left({ }^{0} \mathbf{x}_{(j)}-{ }^{0} \mathbf{x}_{(k)}, t\right)=\left\{\begin{array}{l}1 \text { if interaction exists, } \\ 0 \text { if interaction is broken }\end{array}\right.$

Therefore, by introducing the damage parameter $\psi_{(k)(j)}$, the PD equations of motion in local and global coordinate systems given in Eq. (39) and Eq. (48b), respectively, can be rewritten as

$$
\begin{aligned}
& \mathbf{m}_{(k)}^{L}\left({ }^{t} \ddot{\mathbf{u}}_{(k)}^{L}\right)=\sum_{j=1}^{N} \psi_{(k)(j)}\left({ }_{0}^{t} \mathbf{f}_{(k)(j)}^{L}\right){ }^{0} V_{(j)}+{ }^{t} \mathbf{b}_{(k)}^{L} \\
& \mathbf{m}_{(k)}^{G}\left({ }^{t} \ddot{\mathbf{u}}_{(k)}^{G}\right)=\sum_{j=1}^{N} \psi_{(k)(j)}\left({ }_{0}^{t} \mathbf{f}_{(k)(j)}^{G}\right)^{0} V_{(j)}+{ }^{t} \mathbf{b}_{(k)}^{G}
\end{aligned}
$$

The ratio of eliminated interactions to the total number of interactions associated with a material point within its horizon is defined as the local damage, $\varphi$. This local damage can be represented as [12]

$$
\varphi_{(k)}=1-\frac{\sum_{j=1}^{N} \psi_{(k)(j)}{ }^{0} V_{(j)}}{\sum_{j=1}^{N}{ }^{0} V_{(j)}}
$$

To decide the state of interaction as described in Eq. (50), two common criteria based on the critical bond stretch $[12,15]$ or the critical energy release rate $[23,25,70]$ are used. As presented in the previous section, the deformations of the beam include axial, shear, bending, and torsional components. Therefore, in this study, the damage criterion based on the critical energy release rate is used. This criterion for a beam can be described as [21, 23, 25, 35, 66, 71]

${ }_{0}^{t} \bar{g}_{(k)(j)}<g_{c} \rightarrow$ interaction exists: $\psi_{(k)(j)}=1$

${ }_{0}^{t} \bar{g}_{(k)(j)} \geq g_{c} \rightarrow$ interaction is broken: $\psi_{(k)(j)}=0$

where $g_{c}$ represents the critical energy release rate for one interaction can be obtained by the relation suggested by Madenci and Oterkus [23], [25] as

$$
g_{c}=\frac{G_{c}}{N_{c}}
$$

where $G_{c}$ represents the critical energy release rate for material and $N_{c}$ represents the total number of interactions passing through a unit crack surface which is equal to the cross-section of the beam. According to Nguyen and Oterkus [21], [66], for a PD beam model with a horizon size of $\delta=3.015 \Delta x$ in which $\Delta x$ is the mesh size, this number can be defined as $N_{c}=12$.

In Eq. (53), ${ }_{0}^{t} \bar{g}_{(k)(j)}$ represents the energy release rate for interaction between material points $k$ and $j$ which can be calculated as

$$
{ }_{0}^{t} \bar{g}_{(k)(j)}=\frac{1}{2}\left({ }_{0}^{t} g_{(k)(j)}+{ }_{0}^{t} g_{(j)(k)}\right)
$$


with

$$
\begin{aligned}
& { }_{0}^{t} g_{(k)(j)}=\frac{1}{{ }^{0} A}\left({ }_{0}^{t} \Phi_{(k)(j)}\right)\left({ }^{0} V_{(k)}\right)\left({ }^{0} V_{(j)}\right) \\
& { }_{0}^{t} g_{(j)(k)}=\frac{1}{{ }^{0} A}\left({ }_{0}^{t} \Phi_{(j)(k)}\right)\left({ }^{0} V_{(k)}\right)\left({ }^{0} V_{(j)}\right)
\end{aligned}
$$

where ${ }_{0}^{t} \Phi_{(k)(j)}$ and ${ }_{0}^{t} \Phi_{(j)(k)}$ represent micropotentials of the interaction between material points $k$ and $j$. For the bond-based PD model, ${ }_{0}^{t} \Phi_{(k)(j)}$ and ${ }_{0}^{t} \Phi_{(j)(k)}$ are equal to each other. By applying the same idea introduced by Madenci and Oterkus [23], [25], the micropotentials ${ }_{0}^{t} \Phi_{(k)(j)}$ and ${ }_{0}^{t} \Phi_{(j)(k)}$ for one interaction can be calculated for the bond-based PD model as

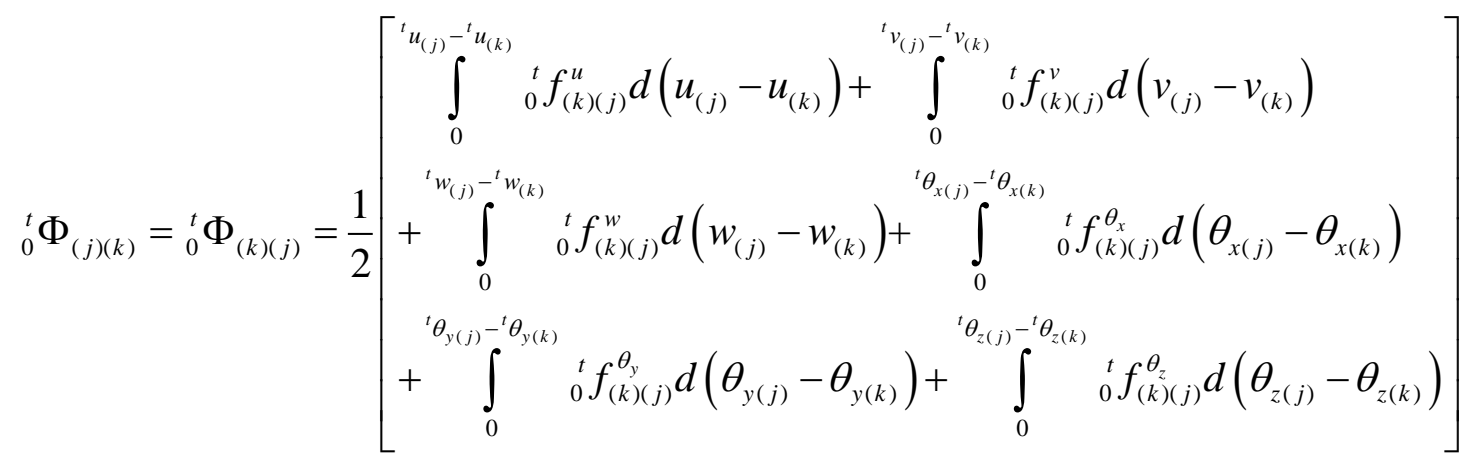

where ${ }_{0}^{t} f_{(k)(j)}^{u},{ }_{0}^{t} f_{(k)(j)}^{v},{ }_{0}^{t} f_{(k)(j)}^{w},{ }_{0}^{t} f_{(k)(j)}^{\theta_{x}},{ }_{0}^{t} f_{(k)(j)}^{\theta_{y}},{ }_{0}^{t} f_{(k)(j)}^{\theta_{z}}$ are given in Eq. (38g-h).

\section{Numerical implementation}

In peridynamics, the numerical integration over the horizon of a material point is approximated by considering the entire volumes of its family members. However, as shown in Fig. 5, material points, shown in red, are not fully inside the horizon of material point $k$, shown in blue. Therefore, the volume correction factors can be applied for these material points to improve the accuracy of the PD calculations $[15,72]$. The volume correction factor, $v_{(k)(j)}$, for the interaction between material points $k$ and $j$ can be calculated as $[15,72]$

$$
v_{(k)(j)}=\left\{\begin{array}{lll}
1 & \text { if } & { }^{0} \xi_{(k)(j)} \leq(\delta-\Delta x / 2) \\
\left(\delta+\Delta x / 2-{ }^{0} \xi_{(k)(j)}\right) / \Delta x & \text { if } & (\delta-\Delta x / 2)<{ }^{0} \xi_{(k)(j)} \leq \delta \\
0 & \text { if } & { }^{0} \xi_{(k)(j)}>\delta
\end{array}\right.
$$

where $\Delta x$ represents the uniform mesh size in the discretized model.

Moreover, as shown in Fig. 5, material points located far from the boundaries of the beam such as material point $k$, shown in blue, have a full horizon with all of its family members. However, material points located near the boundaries of the beam, such as material points 1 and $N$, shown in purple in Fig. 5, have truncated horizons. Therefore, the surface correction factors need to be applied for material points near the boundaries of the beam to improve the PD calculations $[8,16]$. The surface correction factor for the interaction between material points $k$ and $j$ can be approximated as [23]

$$
\Omega_{(k)(j)}=\frac{1}{2}\left(\Omega_{(k)}+\Omega_{(j)}\right)
$$


where $\Omega_{(k)}$ and $\Omega_{(j)}$ represent surface correction factors for material points $k$ and $j$, respectively. The values of the surface correction factors can be determined by comparing the strain energy density in classical continuum mechanics with the strain energy density in peridynamics $[15,57]$. By applying the method proposed by Diyaroglu, et al. [57], the surface correction factors for a material point in a PD discretized model with horizon size of $\delta=3.015 \Delta x$ can be determined as

$\Omega_{(k)}=\left\{\begin{array}{lll}2.000 & \text { if } & n_{\mathrm{F}(k)}=3 \\ 1.639 & \text { if } & n_{\mathrm{F}(k)}=4 \\ 1.205 & \text { if } & n_{\mathrm{F}(k)}=5 \\ 1.000 & \text { if } & n_{\mathrm{F}(k)}=6\end{array}\right.$

where $n_{\mathrm{F}(k)}$ represents the number of family members of material point $k$.

By using the volume and surface correction factors given in Eq. (57) and Eq. (58), the PD equations of motion in local and global coordinate systems given in Eq. (51a) and Eq. (51b), respectively, can be rewritten as

$$
\begin{aligned}
& \mathbf{m}_{(k)}^{L}\left({ }^{t} \ddot{\mathbf{u}}_{(k)}^{L}\right)=\sum_{j=1}^{N} \psi_{(k)(j)}\left({ }_{0}^{t} \mathbf{f}_{(k)(j)}^{L}\right) v_{(k)(j)} \Omega_{(k)(j)}{ }^{0} V_{(j)}+{ }^{t} \mathbf{b}_{(k)}^{L} \\
& \mathbf{m}_{(k)}^{G}\left({ }^{t} \ddot{\mathbf{u}}_{(k)}^{G}\right)=\sum_{j=1}^{N} \psi_{(k)(j)}\left({ }_{0}^{t} \mathbf{f}_{(k)(j)}^{G}\right) v_{(k)(j)} \Omega_{(k)(j)}{ }^{0} V_{(j)}+{ }^{t} \mathbf{b}_{(k)}^{G}
\end{aligned}
$$

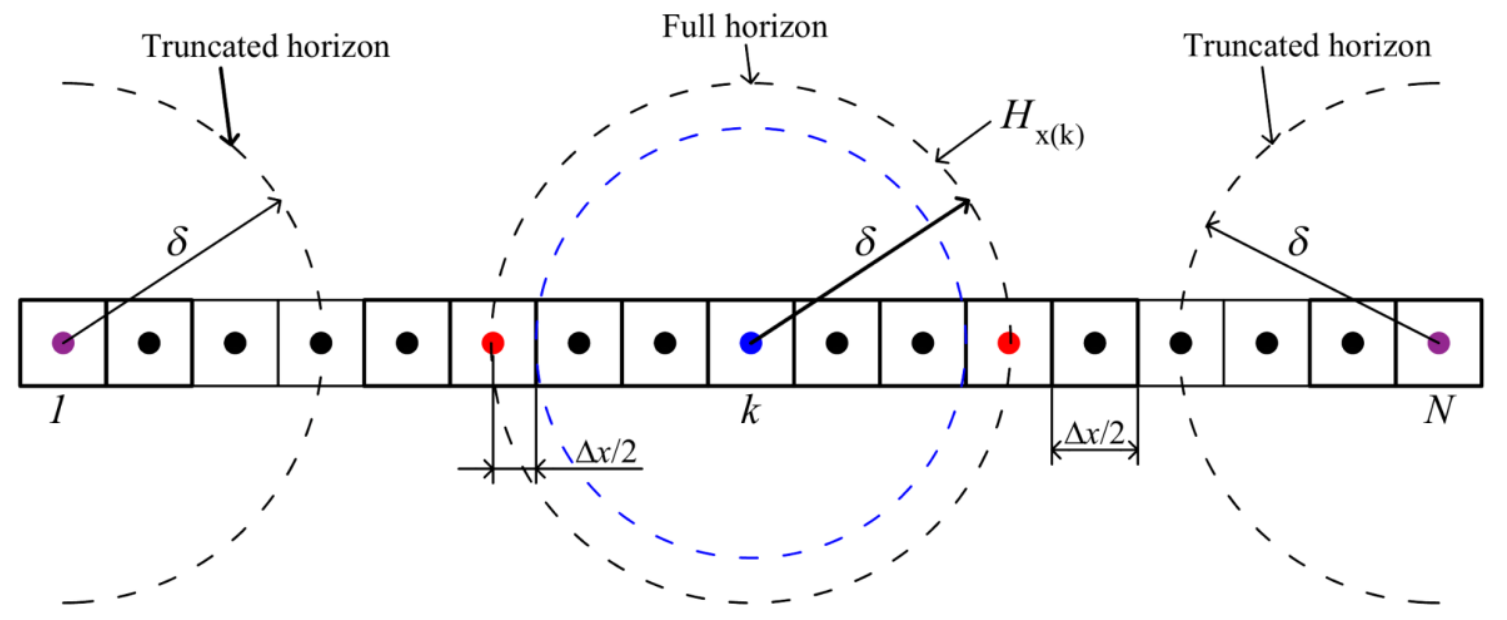

Fig. 5. Volume and surface corrections

In the PD model, the structure is uniformly discretized with material points associated with specific volumes. The director vectors with respect to the global coordinates are primarily defined for undeformed configuration. The PD equation of motion can be solved by using a meshless scheme. For static problems, time integration can be achieved through an explicit scheme using the adaptive dynamic relaxation method (ADR) described by Kilic and Madenci [67]. The fictitious time step size used for ADR solution is $\mathrm{dt}=1[15,67,73]$. In this case, the real mass vector of material points, in Eq. (48b) or (51b) can be replaced by a fictitious mass stable vector as presented in Eq. (B.2). The value of this mass stable vector is chosen to ensure a converged solution as presented 
in Appendix B. The numerical procedure for nonlinear analysis of beam structures is shown in Fig.

6.

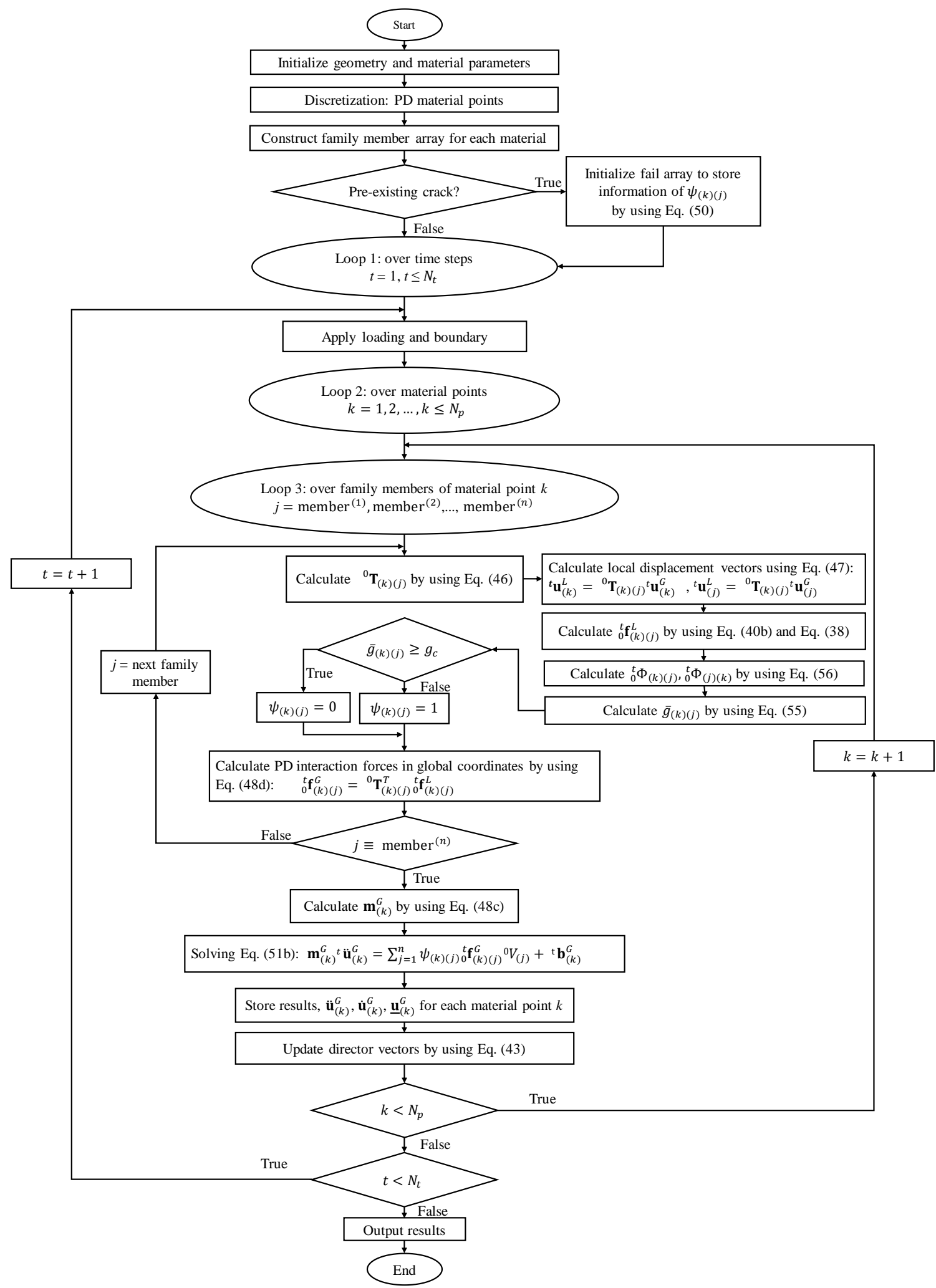

Fig. 6. Numerical procedure for geometrically nonlinear analysis of beam structure in PD 


\section{Numerical results}

In this section, large deformations of beam structures are predicted. For verification purposes, the results predicted by the proposed nonlinear PD model are compared with nonlinear FEA results in Section 7.1. After verifying the accuracy of the nonlinear PD model, damages on a beam subjected to bending and torsional loading conditions are predicted in Section 7.2.

\section{1. $P D$ verification}

In this section, first, large deformations of a cantilever beam subjected to static loading are presented in Section 7.1.1. Next, the nonlinear analyses for curved beams are presented in Section 7.1.2 and 7.1.3. In nonlinear PD analyses, the horizon size of $\delta=3.015 \Delta x$, in which $\Delta x$ represents the mesh size, is used. The adaptive dynamic relaxation (ADR) method [73] is used for these static loading conditions [67] as described in Appendix B. The nonlinear FEA is conducted by using the BEAM188 element in ANSYS.

\subsubsection{A cantilever beam subjected to static loading}

In this section, a cantilever beam with a length of $L=1 \mathrm{~m}$ and a square cross-section of $b=h=0.1 \mathrm{~m}$ is investigated as shown in Fig. 7. The beam has an elastic modulus of $E=2 \times 10^{11} \mathrm{~N} / \mathrm{m}^{2}$ and shear modulus of $G=1 \times 10^{11} \mathrm{~N} / \mathrm{m}^{2}$. The shear correction factor for a square cross-section is $k_{s}=5 / 6$ [7].

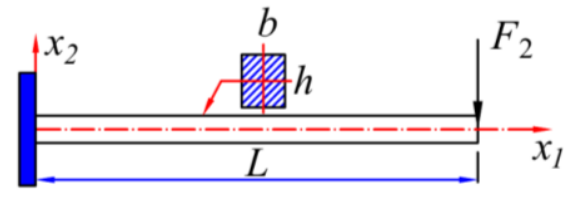

(a)

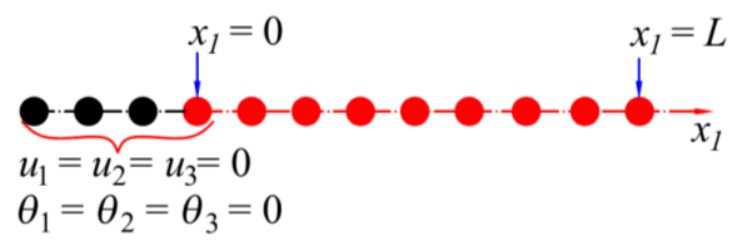

(b)

Fig. 7. Cantilever beam: (a) geometry, (b) PD model discretization and boundary conditions

The beam is fixed on the left end and it is subjected to a transverse force $F_{2}=-8 \times 10^{6} \mathrm{~N}$ in $x_{2}$ direction at the right end as shown in Fig. 7(a). In the PD model, the beam is discretized with a mesh size of $\Delta x=L / 100$. In the FEA, the same mesh size is used.

To apply boundary conditions in PD, three fictitious material points, shown in black in Fig. 7(b), are added on the left end of the beam. All degrees of freedom of these fictitious material points and the material point located at $x_{1}=0$ are set equal to zero. To apply loading condition, the material point at the right end of the beam is subjected to a body force as

$b_{2}=F_{2} / V_{(N)}$

where $V_{(N)}$ is the volume of material point located at $x_{1}=L$.

The body force given in Eq. (61) is applied at once and kept constantly during ADR solution. In the explicit time integration scheme using the ADR method, the fictitious time step size is $d t=1$ $[15,67,73]$. 
Fig. 8 shows the convergence of the PD solution using the ARD method. The variations of displacements and rotations of the material points located at $x_{1}=L$ and $x_{1}=L / 2$ were plotted against the iteration numbers as shown in Fig. 8(a) and Fig. 8(b), respectively. As can be seen from the figures, the PD solution converges after 4000 iterations. The ADR method for this study is presented in Appendix B.

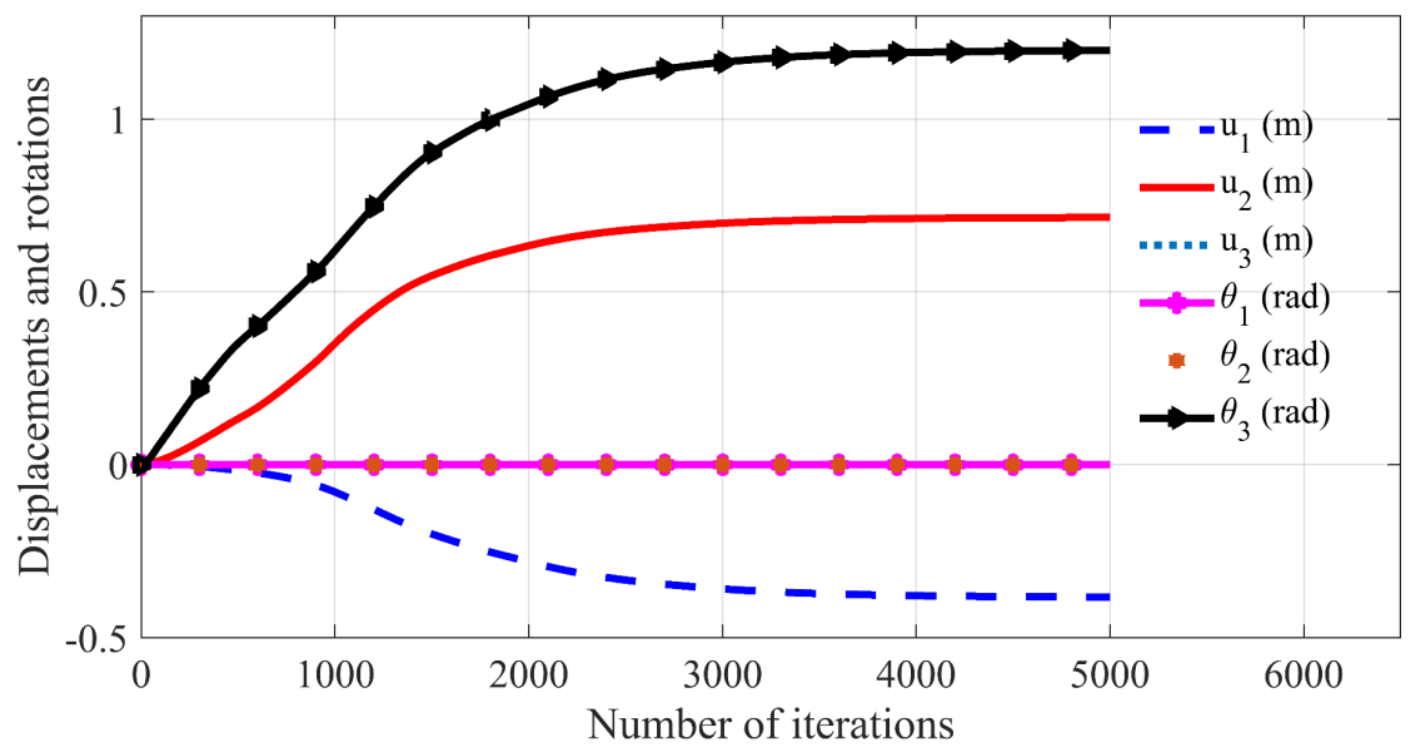

(a)

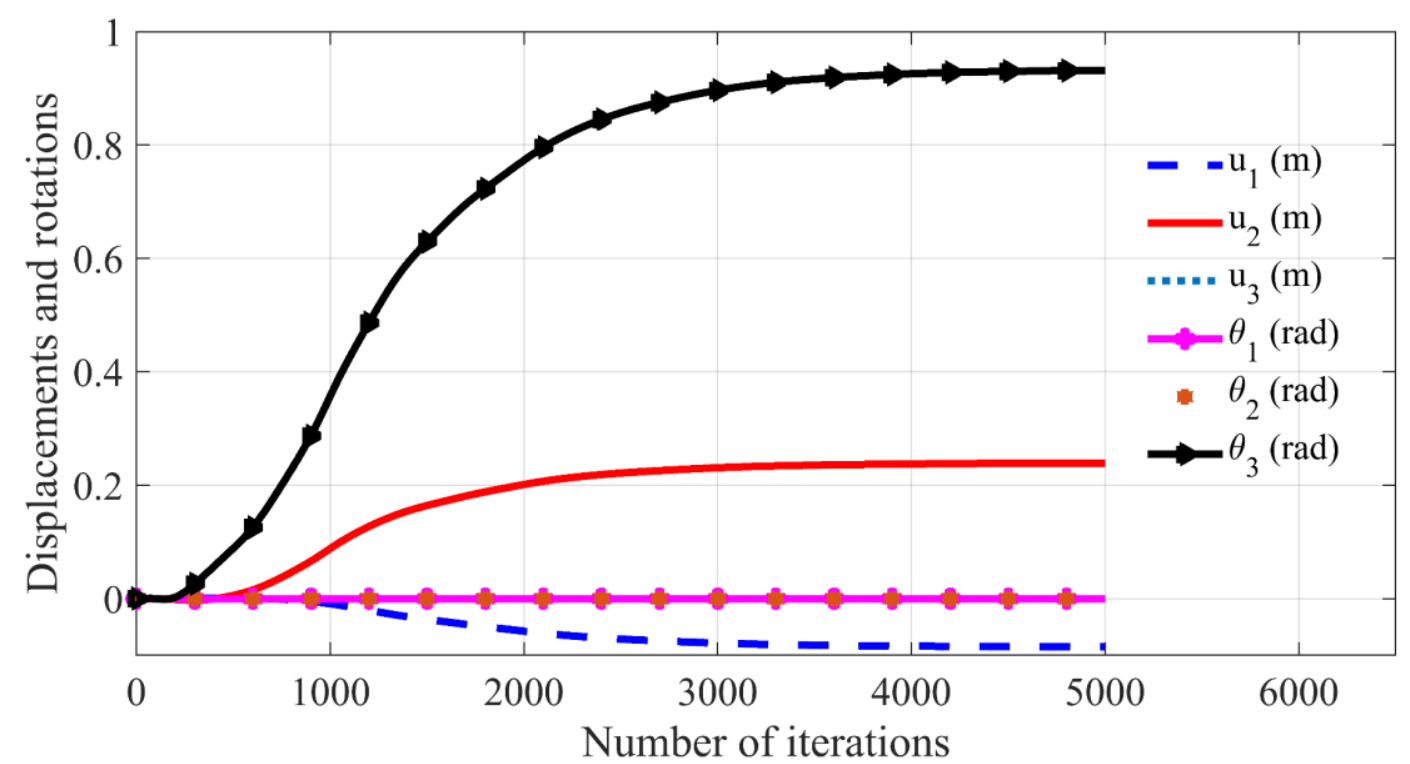

(b)

Fig. 8. The convergence of displacements and rotations in the PD simulation of (a): a material point located at $x_{1}=L,(\mathrm{~b})$ : a material point located at $x_{1}=L / 2$

Fig. 9 shows a comparison between nonlinear PD results and FEA results. As shown in Fig. 9(a), the deformed configurations of the beam captured by using the nonlinear PD model and nonlinear FEA match very well, meanwhile the linear FEA gives a completely different deformed configuration. As can be seen from Fig. 9(b), the linear FEA results show that the beam does not 
have axial deformations when it is subjected to transverse force. On the contrary, both nonlinear PD and nonlinear FEA results show that the beam has nonzero axial deformations due to the coupling of the axial, shear, and bending deformations. As shown in Fig. 9(c-d), the nonlinear PD and nonlinear FEA solutions have a very good agreement on $u_{2}$ and $\theta_{3}$, meanwhile, the linear FEA gives much larger values.

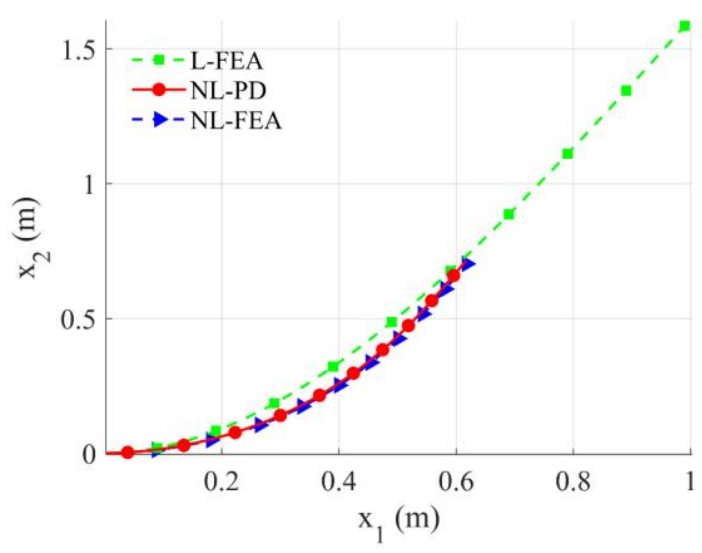

(a)

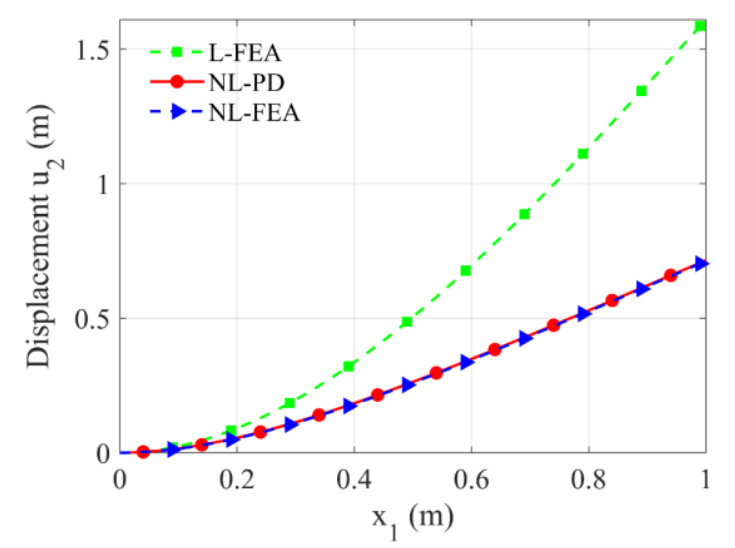

(c)

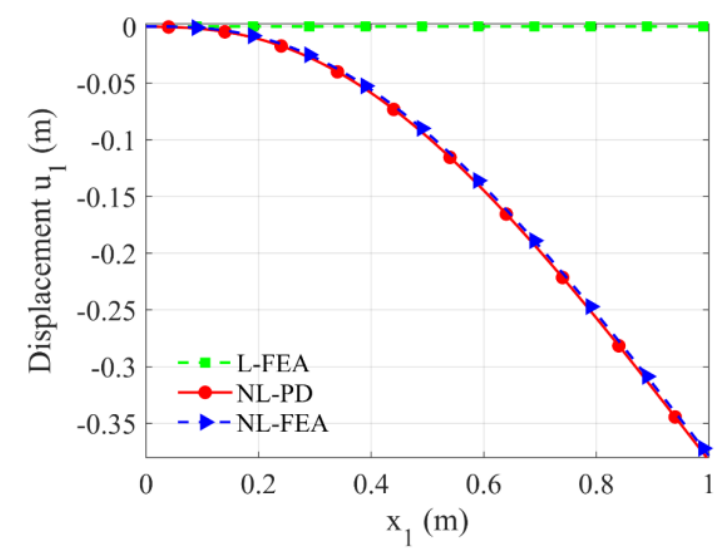

(b)

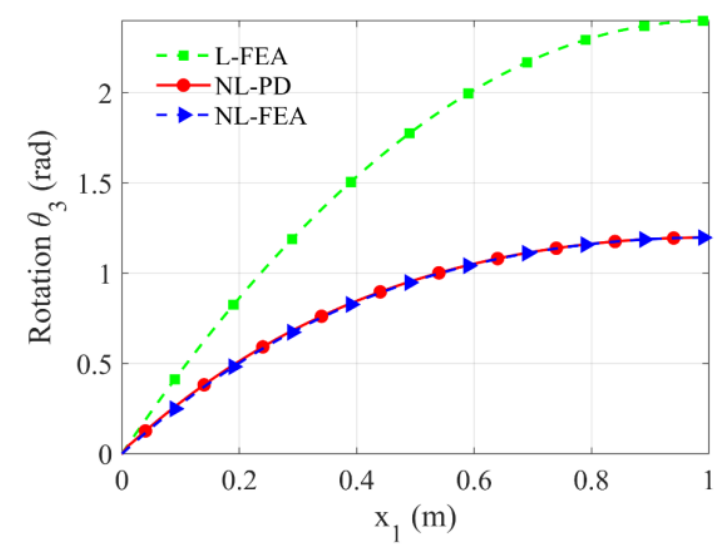

(d)

Fig. 9. The deformation of the beam subjected to a transverse end force $F_{2}=-8 \times 10^{6} \mathrm{~N}$ (a) deformed configurations, (b) displacements $u_{1}$, (c) displacement $u_{2}$, (d) rotation $\theta_{3}$ (L represents linear; NL represents nonlinear)

To further verify the nonlinear PD model for straight beams, a parametric study for the beam subjected to various transverse forces, $F_{2}=-n \times 10^{6} \mathrm{~N}$ with $n=2,4, \ldots, 18,20$, is investigated as shown in Fig. 10. As can be observed from Fig. 10(a), the deformed configurations of the beam captured by the nonlinear PD model match very well with those captured by nonlinear FEA. Fig. 10 (b) shows the variations of nonzero degrees of freedom, $u_{1}, u_{2}$ and $\theta_{3}$, on the right tip of the beam. As can be seen from the figure, the nonlinear PD and nonlinear FEA results agree very well which shows the accuracy of the nonlinear PD model for straight beams. 


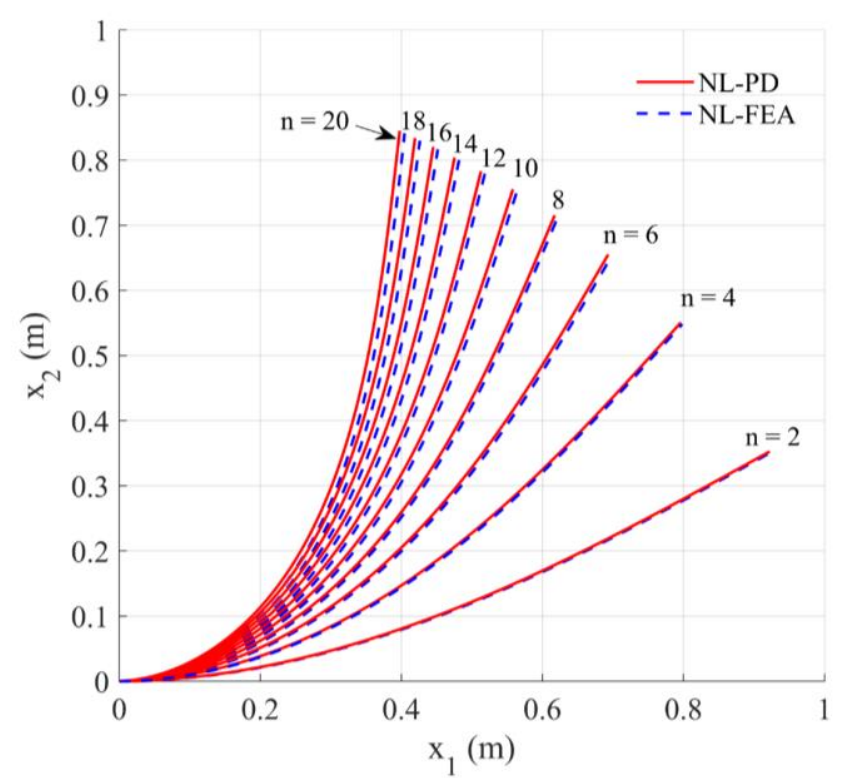

(a)

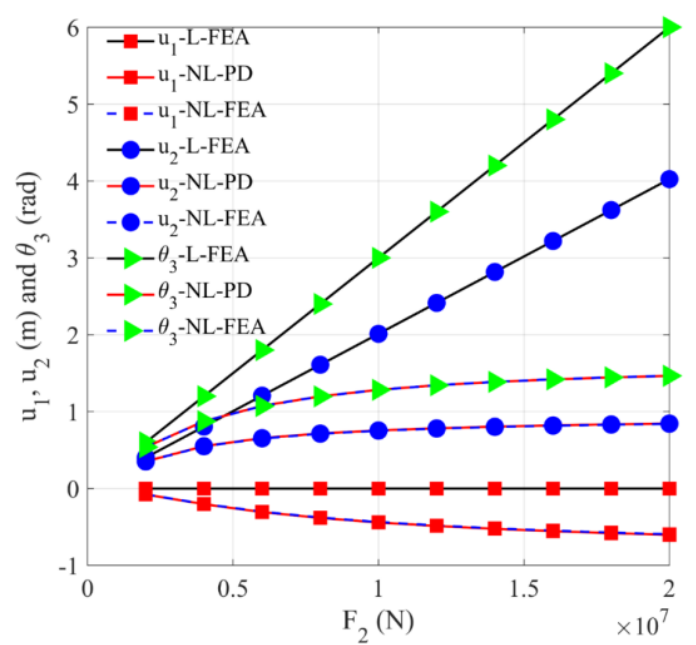

(b)

Fig. 10. Deformations of the beam subjected to a transverse force $F_{2}=-n \times 10^{6} \mathrm{~N}$ with $n=2 \div 20$ (a) deformed configurations, (b) displacements and rotation of the right end ( $\mathrm{L}$ represents linear; NL represents nonlinear)

To further verify the PD model, another parametric study for the beam subjected to various bending moments $M_{2}=n \times 2 \pi E I_{22} / L$ with $n=1,3 / 4,1 / 2,1 / 3,1 / 4,1 / 5,1 / 10,1 / 20$ on the right end is investigated as shown in Fig. 11. As can be observed from the figure, the deformed configurations of the beam captured by the nonlinear PD model agree very well with the nonlinear FEA results studied by Pai and Palazotto [74]. Therefore, the accuracy of the nonlinear PD for the straight beams is verified.

Note that, in this study, the director vectors of the beam are updated at each iteration by using Eq. (43) as shown in Fig. 6. Therefore, the beam which is initially straight can become a curved beam after several iterations. Therefore, the developed nonlinear PD model based on the Total Lagrangian formulations still can capture the behaviours of the beam as shown in Fig. 11. 


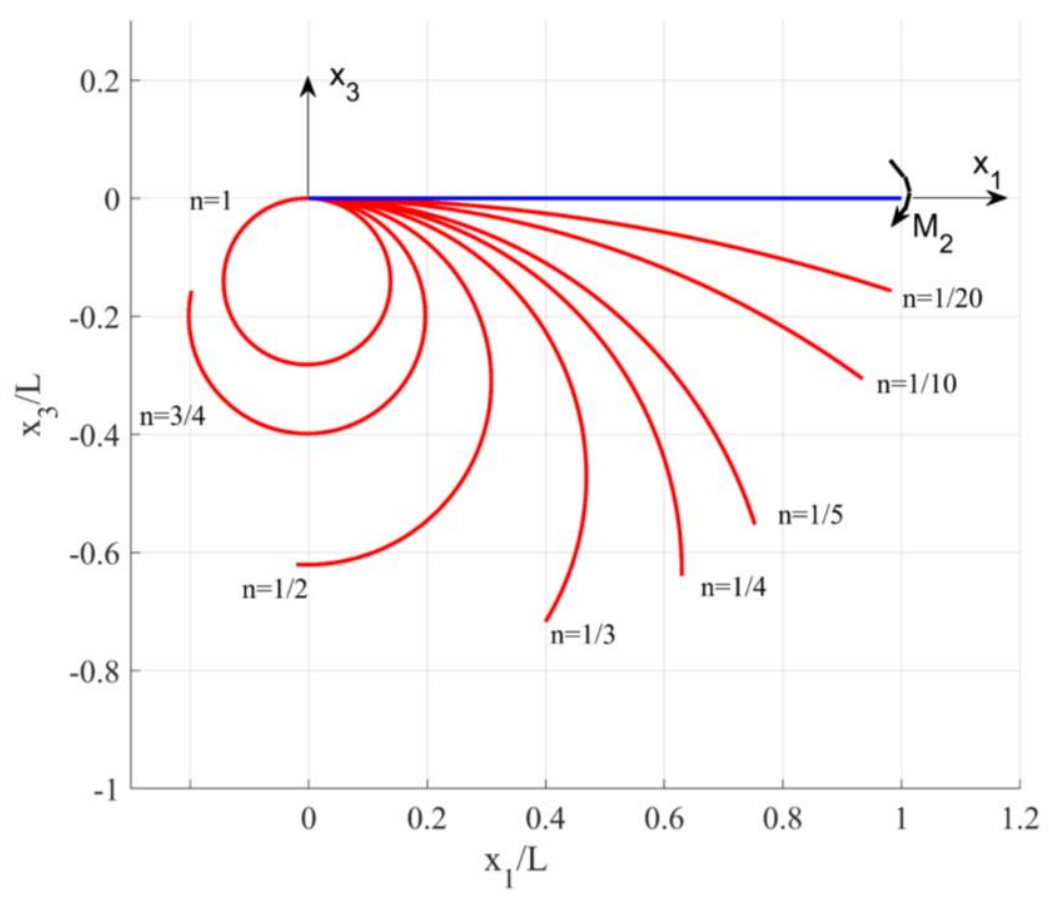

Fig. 11. Deformed configurations of the cantilever beam subjected to bending moment

$$
M_{2}=n \times 2 \pi E I_{22} / L
$$

7.1.2. A half-circular beam subjected to tangential end load

In this section, a half-circular beam subjected to tangential end loads is investigated as shown in Fig. 12. The beam has a radius of $R=0.127 \mathrm{~m}$ and a rectangular cross-section with $b=6.35 \times 10^{-3} \mathrm{~m}$ and $h=b / 3$ [74]. The beam has an elastic modulus of $E=6.895 \times 10^{10} \mathrm{~N} / \mathrm{m}^{2}$ and shear modulus of $G=2.6518 \times 10^{10} \mathrm{~N} / \mathrm{m}^{2}$. For the rectangular cross-section, the shear correction factor is chosen as $k_{s}=5 / 6$ [7].

The beam is fixed at $\left(x_{1}=0, x_{2}=0, x_{3}=0\right)$ and subjected to a tangential force $F_{1}=n E I_{22} / R^{2}$ with $n=1,0.8,0.6,0.5,0.4,0.3,0.2,0.1$ in $x_{1}$ direction at $\left(x_{1}=0, x_{2}=0, x_{3}=-2 R\right)$. In the PD model, the beam is discretized with a mesh size of $\Delta x=\pi R / 300$. In FEA, the same mesh size is used. To apply the boundary conditions in PD, three fictitious material points are added on the fixed end and all DOFs of these points, as well as DOFs of the material point located at $\left(x_{1}=0, x_{2}=0, x_{3}=0\right)$ are set equal to zero. 


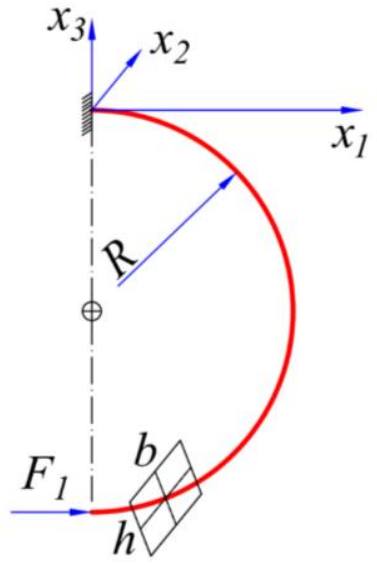

(a)

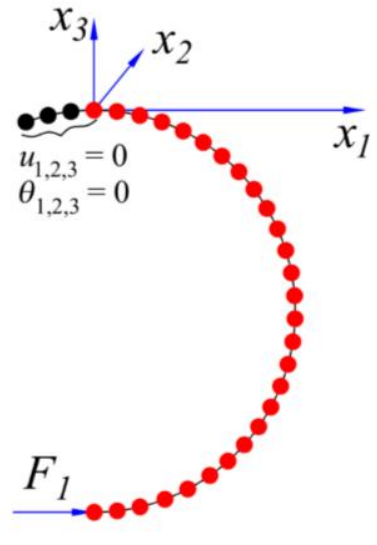

(b)

Fig. 12. A half-circular beam subjected to tangential load: (a) geometry; (b) PD model discretization and boundary conditions

Fig. 13 shows the comparison between nonlinear PD and nonlinear FEA results. Fig. 13(a) shows the deformed configurations for the beam in all loading conditions. Fig. 13(b) shows the deflection versus $n$ curve of the right tip. It can be observed from the figures that the nonlinear PD and nonlinear FEA results have a very good agreement and both results agree very well with those captured by Pai and Palazotto [74].

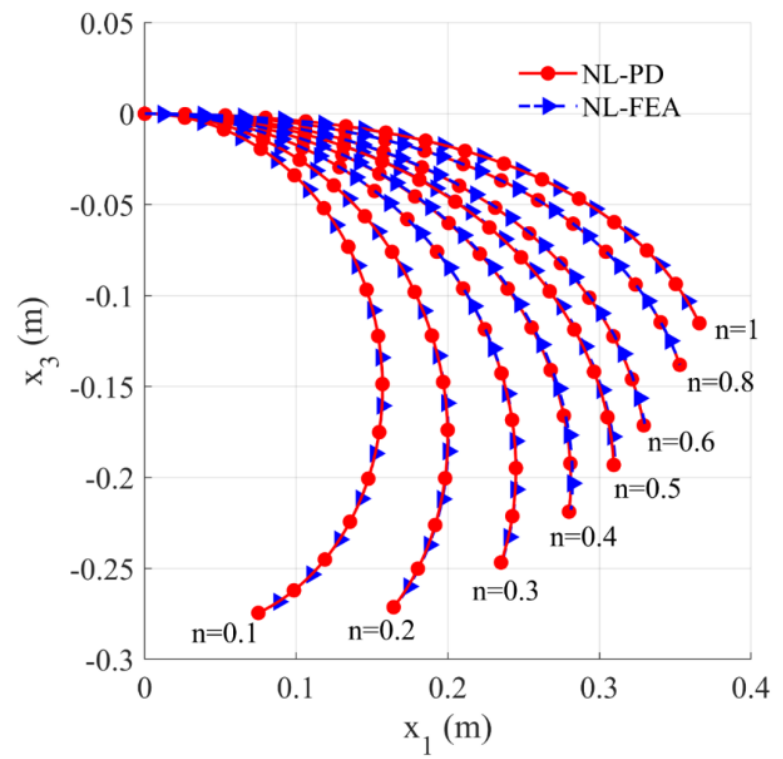

(a)

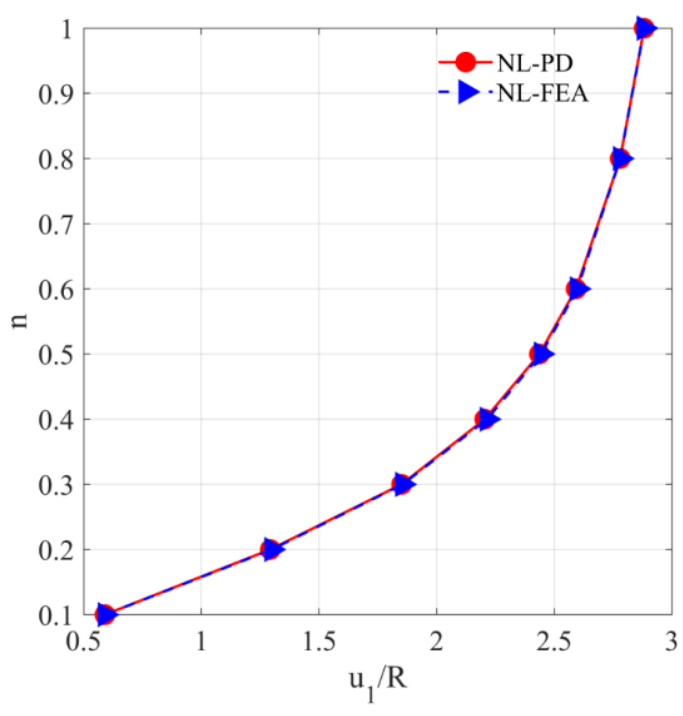

(b)

Fig. 13. The deformations of a half-circular beam subjected to end force $F_{1}=n E I_{22} / R^{2}$ (a) the deformed configurations, (b) the deflection versus $n$ curve of the right tip (NL represents nonlinear) 


\subsubsection{Large displacements in the 3D analysis of a 45-degree curved beam}

In this section, a 45-degree curved beam subjected to end load is investigated as shown in Fig. 14. The beam has the radius of $R=2.54 \mathrm{~m}$ and a square cross-section with $b=h=0.0254 \mathrm{~m}$. The beam has the elastic modulus of $E=6.895 \times 10^{10} \mathrm{~N} / \mathrm{m}^{2}$ and shear modulus of $G=3.4474 \times 10^{10} \mathrm{~N} / \mathrm{m}^{2}$. The beam is investigated in two loading conditions with $F_{3}=1334.4665 \mathrm{~N}$ and $F_{3}=2668.933 \mathrm{~N}$ [75]. For the square cross-section, the shear correction factor is chosen as $k_{s}=5 / 6$ [7], and the torsional constant can be chosen as $k_{t}=0.1406 \times b^{4}$ [69]

In the PD model, the beam is discretized into 300 material points. Similar to previous analyses, three fictitious material points are added on the fixed end as shown in Fig. 14(b). All degrees of freedom of these fictitious points, as well as the material point located at $\left(x_{1}=0, x_{2}=0, x_{3}=0\right)$ are set equal to zero.

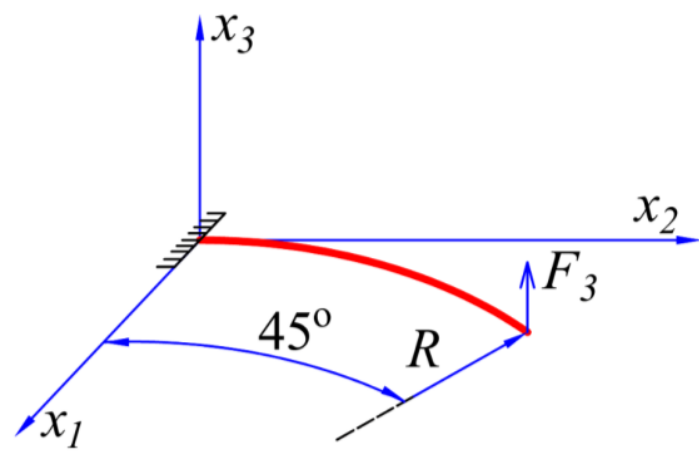

(a)

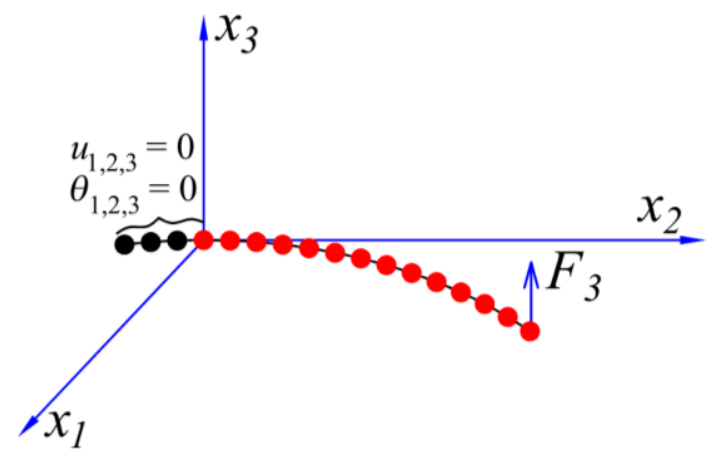

(b)

Fig. 14. A 45-degree curved beam (a) geometry; (b) PD discretized model

Fig. 15 shows the deformed configurations of the beam. It can be observed from the figure that nonlinear PD and nonlinear FEA results have good agreement in both loading conditions. Table 1 shows the locations of the free end of the beam in two loading conditions. As can be seen from Table 1, the nonlinear PD results agree well with both nonlinear FEA results by using ANSYS and results studied by Bathe and Bolourchi [75]. Therefore, the accuracy of the nonlinear PD model is verified. 


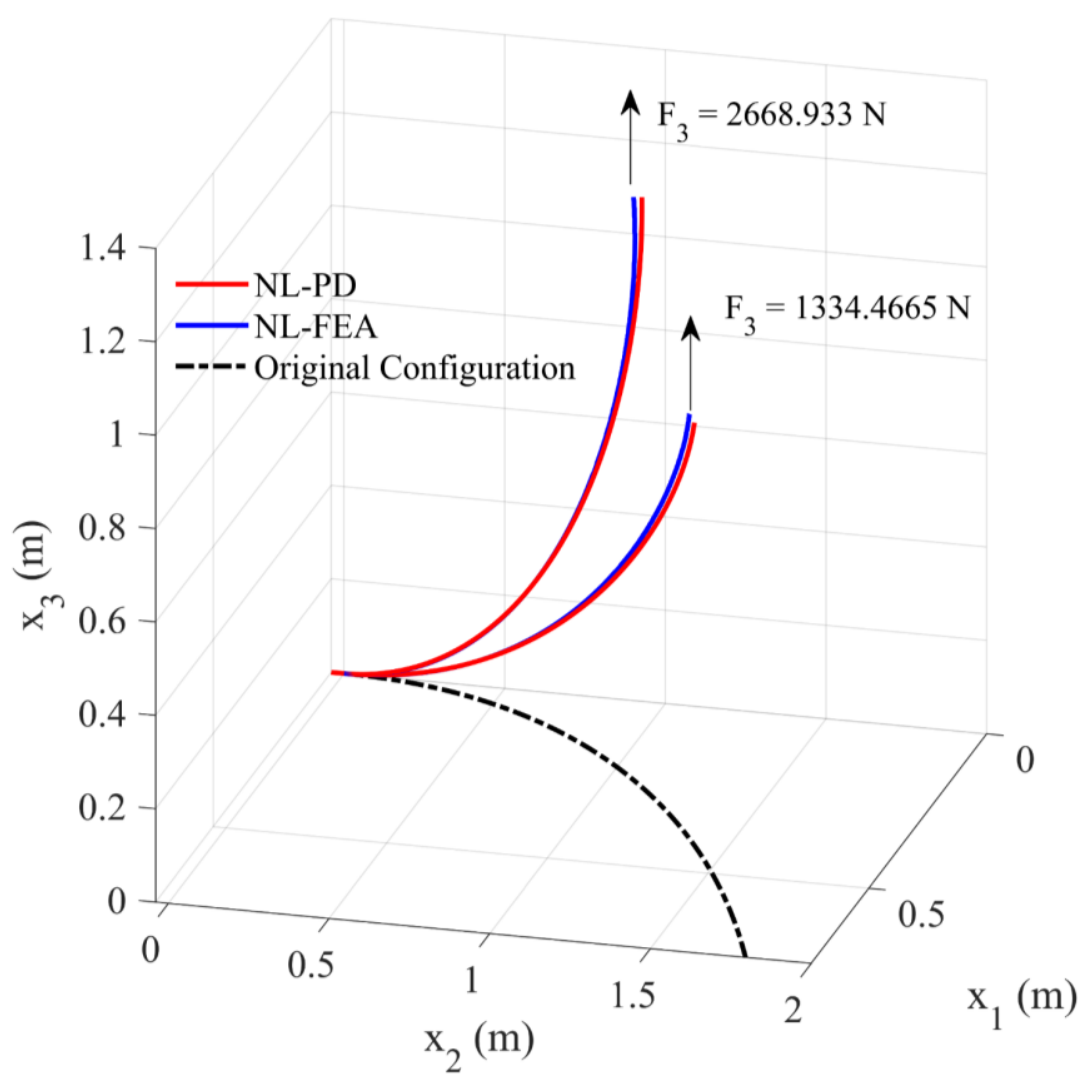

Fig. 15. Deformed configurations of the 45 -degree curved beam

Table 1. Positions of the right tip with different applied force values

\begin{tabular}{|c|c|c|c|}
\hline & \multicolumn{3}{|c|}{ Right tip location $\left(x_{1}, x_{2}, x_{3}\right)$ in meters } \\
\hline $\begin{array}{c}\text { Applied force } \\
F_{3}(\mathrm{~N})\end{array}$ & $\begin{array}{c}\text { Nonlinear PD } \\
(200 \text { elements })\end{array}$ & $\begin{array}{c}\text { Nonlinear ANSYS } \\
(200 \text { elements })\end{array}$ & $\begin{array}{c}\text { Bathe and Bolourchi [75] } \\
(4 \text { elements })\end{array}$ \\
\hline 1334.467 & $(0.565,1.505,1.008)$ & $(0.563,1.489,1.025)$ & $(0.5715,1.504,1.003)$ \\
\hline 2668.933 & $(0.388,1.212,1.355)$ & $(0.397,1.193,1.360)$ & $(0.404,1.199,1.356)$ \\
\hline
\end{tabular}

\subsection{PD damage prediction}

After verifying the accuracy of the proposed nonlinear PD model, in this section, damages on a dry spaghetti subjected to bending and torsion are predicted. The spaghetti is represented by a straight beam with a length of $L=0.24 \mathrm{~m}$ and it has a solid circular cross-section with an average radius of $r=0.00085 \mathrm{~m}$ as shown in Fig. 16 [76]. In the PD model, the beam is discretized with a mesh size of $\Delta x=L / 600$. To represent the imperfection of the spaghetti microstructure [77], the radius of the beam's cross-section is assumed to vary randomly in a range of $8.4832 \times 10^{-4} \leq r\left(x_{1}\right) \leq 8.5169 \times 10^{-4}(\mathrm{~m})$ as shown in Fig. 16(b). 
The material has a mass density of $\rho=1500 \mathrm{~kg} / \mathrm{m}^{3}$, elastic modulus of $E=3.8 \times 10^{9} \mathrm{~N} / \mathrm{m}^{2}[76]$, the fracture toughness of $K_{I c}=0.478 \mathrm{MPa} \sqrt{\mathrm{m}}$ [77], and the critical energy release rate of $G_{c}=60.1274 \mathrm{~J} / \mathrm{m}^{2}$. The spaghetti is investigated in two loading conditions: pure bending (case 1) and a combination of torsion and bending (case 2) [76].

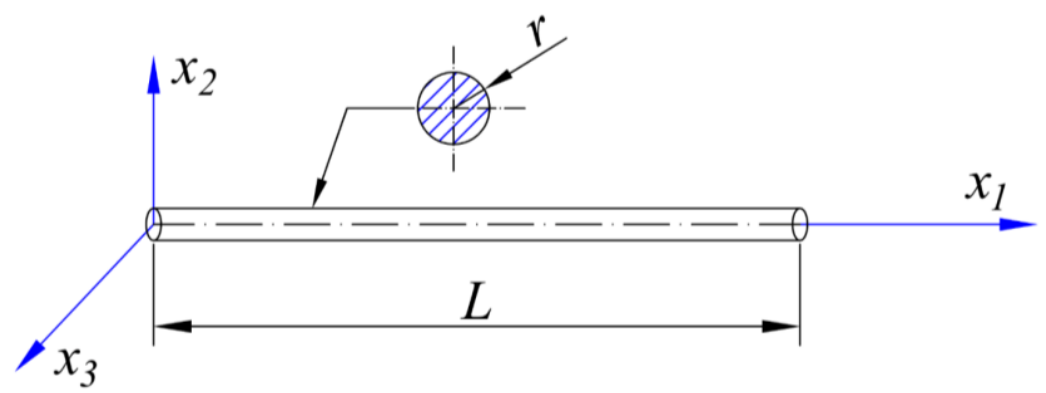

(a)

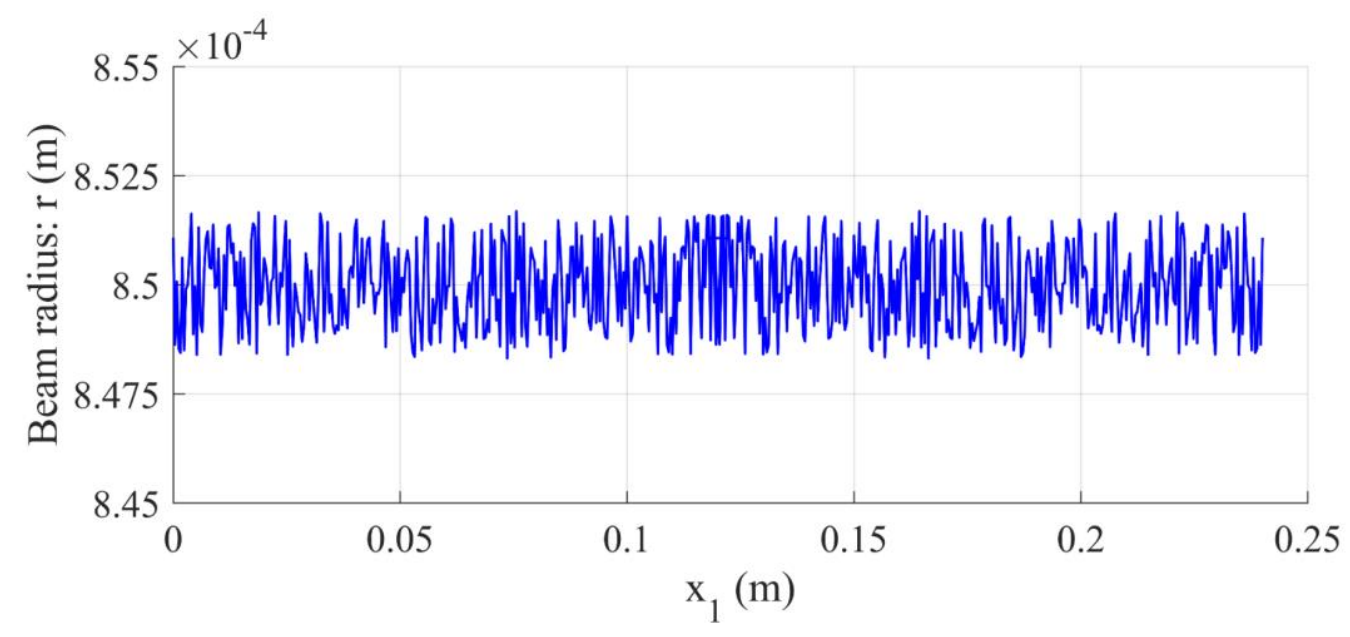

(b)

Fig. 16. A dry spaghetti (a): geometry, (b): randomly variation of cross-section radius along the beam length

\subsubsection{Case 1: Spaghetti subjected to pure bending}

In this case, the beam is subjected to bending moments at two ends as shown in Fig. 17. The boundary and loading conditions applied for the beam include two stages:

(1) Stage 1: the beam is subjected to incremental bending moments while fixing the node at the middle of the beam as shown in Fig. 17(a-b). The bending moments are kept increasing until the first damage occur as shown in Fig. 17(c).

(2) Stage 2: after the first damage occurs at the end of stage 1, the bending moments are released. Then, two ends of the beam are kept at the deformed configuration as shown in Fig. 18 until the final damages occur. 
In stage 1 , the beam is subjected to incremental bending moments per unit volume at two ends as

$$
\begin{aligned}
& { }^{t} m_{3(1)}=\frac{\left({ }^{t-\Delta t} M+\Delta M\right)}{{ }^{0} V_{(1)}} \\
& { }^{t} m_{3(N)}=-\frac{\left({ }^{t-\Delta t} M+\Delta M\right)}{{ }^{0} V_{(N)}}
\end{aligned}
$$

with

${ }^{0} V_{(1)}=\pi r_{(1)}^{2} \Delta x, \quad{ }^{0} V_{(N)}=\pi r_{(N)}^{2} \Delta x$

${ }^{0} M=0, \quad \Delta M=10^{-9} \mathrm{Nm}$

where ${ }^{0} V_{(1)}$ and ${ }^{0} V_{(N)}$ represent volumes of material points located at the left end and right end of the beam, respectively. The term ${ }^{t-\Delta t} M$ represents the applied bending moment at the previous load step, $\Delta M$ represent the incremental value of the bending moment. In Eq. (61c), $r_{(1)}$ and $r_{(N)}$ represent the beam's radius of material points located at the left end and right end, respectively.

Besides, all material points along the beam are also subjected to the gravitational body forces as ${ }^{t} b_{2(k)}=-\rho g$

where $g=9.81 \mathrm{~N} / \mathrm{m}^{2}$ represents the gravitational acceleration.

The boundary conditions for the beam in this stage can be represented as

$$
\begin{aligned}
& u_{2}(0,0,0)=u_{2}(L, 0,0)=0 \\
& u_{3}(0,0,0)=u_{3}(L, 0,0)=0 \\
& u_{1}(L / 2,0,0)=u_{3}(L / 2,0,0)=0
\end{aligned}
$$

In stage 2, when the first damage occurs, the gravitational forces given in Eq. (62) are maintained, meanwhile, the bending moments at the two ends given in Eq. (61) are released as

$$
{ }^{t} m_{3(1)}={ }^{t} m_{3(N)}=0
$$

To apply boundary conditions in the second stage, three material points on each end of the beam are maintained its configurations as the first damage occurs as shown in Fig. 18(b). The boundary conditions for the beam in stage 2 is represented as

$$
\begin{aligned}
& \left({ }^{t} u_{1},{ }^{t} u_{2},{ }^{t} u_{3},{ }^{t} \theta_{1},{ }^{t} \theta_{2},{ }^{t} \theta_{3}\right)=\left({ }^{t_{0}} u_{1},{ }^{t_{0}} u_{2},{ }^{t_{0}} u_{3},{ }^{t_{0}} \theta_{1},{ }^{t_{0}} \theta_{2},{ }^{t_{0}} \theta_{3}\right) \quad \text { at } x_{1} \leq 3 \Delta x \\
& \left({ }^{t} u_{1},{ }^{t} u_{2},{ }^{t} u_{3},{ }^{t} \theta_{1},{ }^{t} \theta_{2},{ }^{t} \theta_{3}\right)=\left({ }^{t_{0}} u_{1},{ }^{t_{0}} u_{2},{ }^{t_{0}} u_{3},{ }^{t_{0}} \theta_{1},{ }^{t_{0}} \theta_{2},{ }^{t_{0}} \theta_{3}\right) \quad \text { at } x_{1} \geq L-3 \Delta x
\end{aligned}
$$

where $t_{0}$ is the moment that the first damage occurs and $\left({ }^{t_{0}} u_{1},{ }^{t_{0}} u_{2},{ }^{t_{0}} u_{3},{ }^{t_{0}} \theta_{1},{ }^{t_{0}} \theta_{2},{ }^{t_{0}} \theta_{3}\right)$ represent displacements and rotations at time $t_{0}$ when the first damage occurs. 


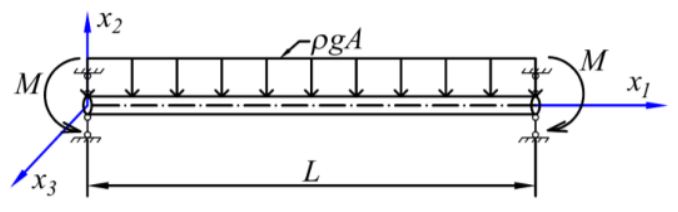

(a)

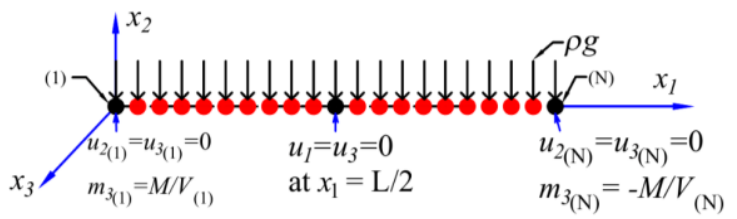

(b)

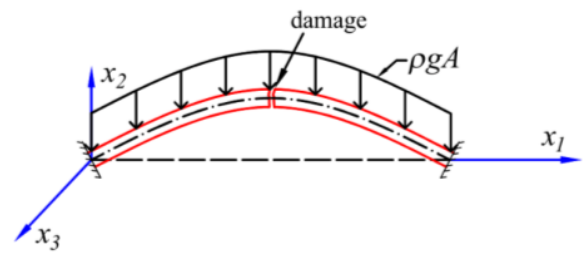

(c)

Fig. 17. Boundary and loading conditions for a spaghetti subjected to pure bending in stage 1 (a): geometry, (b): PD discretized model, (c): deformed configuration at the end of stage 1 when the first damage occurs

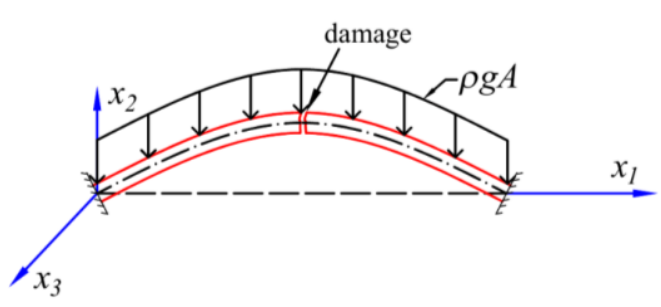

(a)

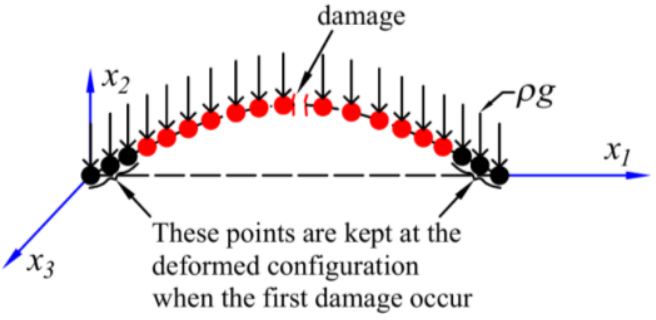

(b)

Fig. 18. Boundary and loading conditions for spaghetti in stage 2 (a): deformed configuration when the first damage occur and the bending moments are released, (b): PD discretized model

The beam is assumed to be in quasi-static loading conditions and the PD solution is obtained by using the ADR method [67, 73]. Fig. 19 shows the damage evolution on the beam in the pure bending condition after stage 1 . As shown in Fig. 19 (a), after $178 \times 10^{5}$ time steps, the first damage occurs at the middle section of the beam. After $178.26 \times 10^{5}$ time steps, in stage 2 the beam has two more damages located symmetrically with respect to the middle section of the beam as shown in Fig. 19(b). After $178.62 \times 10^{5}$ time steps, the beam is finally broken into 6 segments with 5 locations of damage located symmetrically with respect to the middle section of the beam as shown in Fig. 19(c). As it can be observed from the results, the damage evolution on the beam predicted by the nonlinear PD model has good agreement with the experimental results in [76]. 


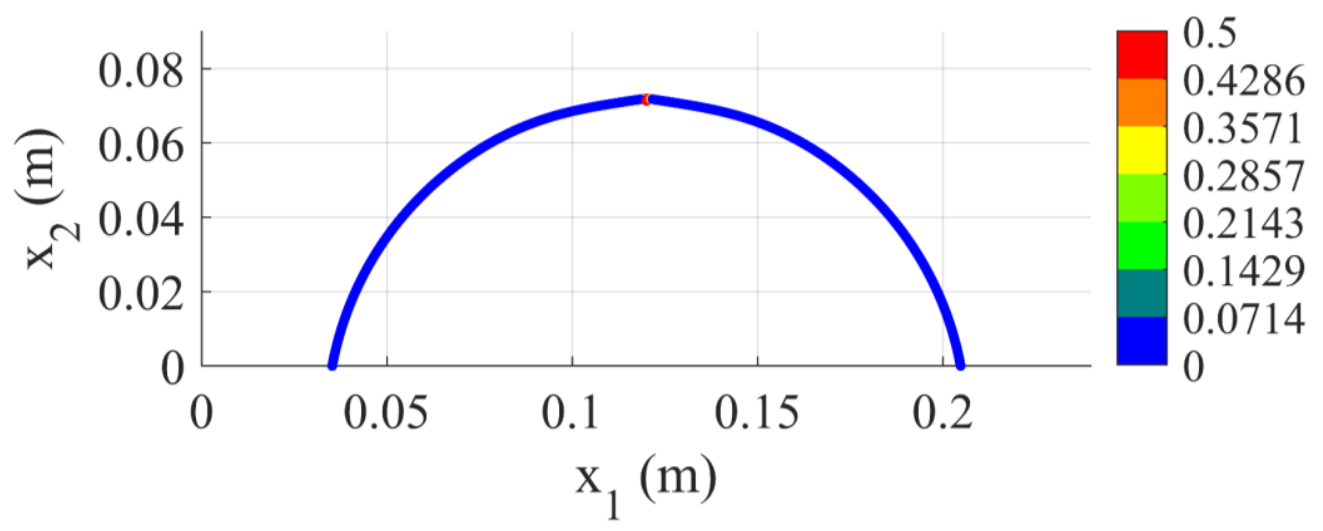

(a)

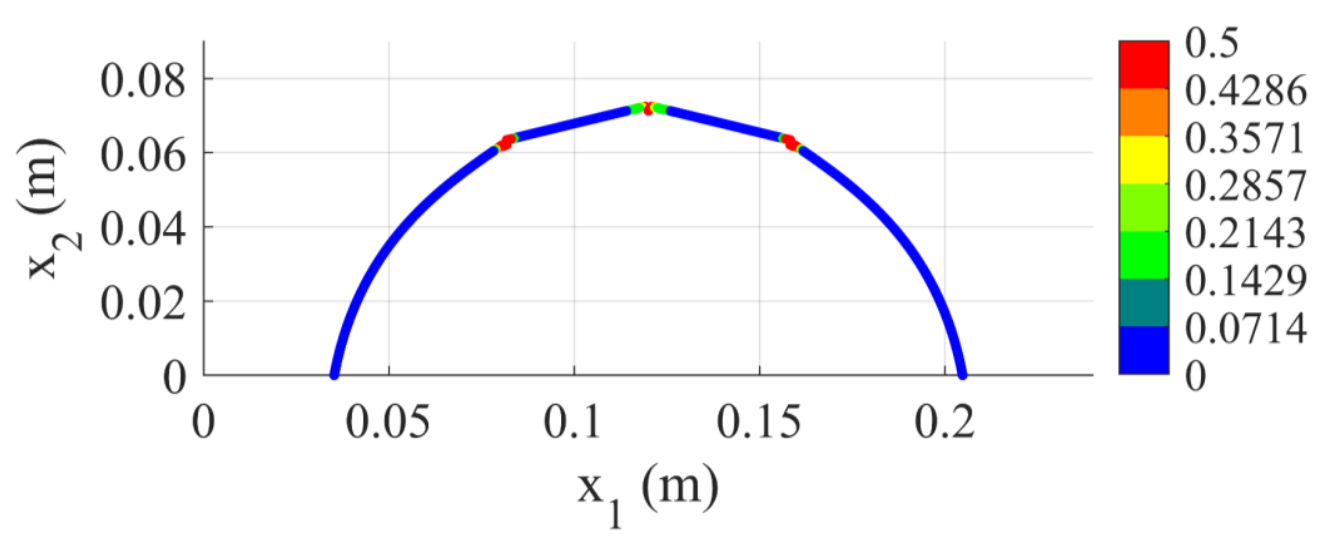

(b)

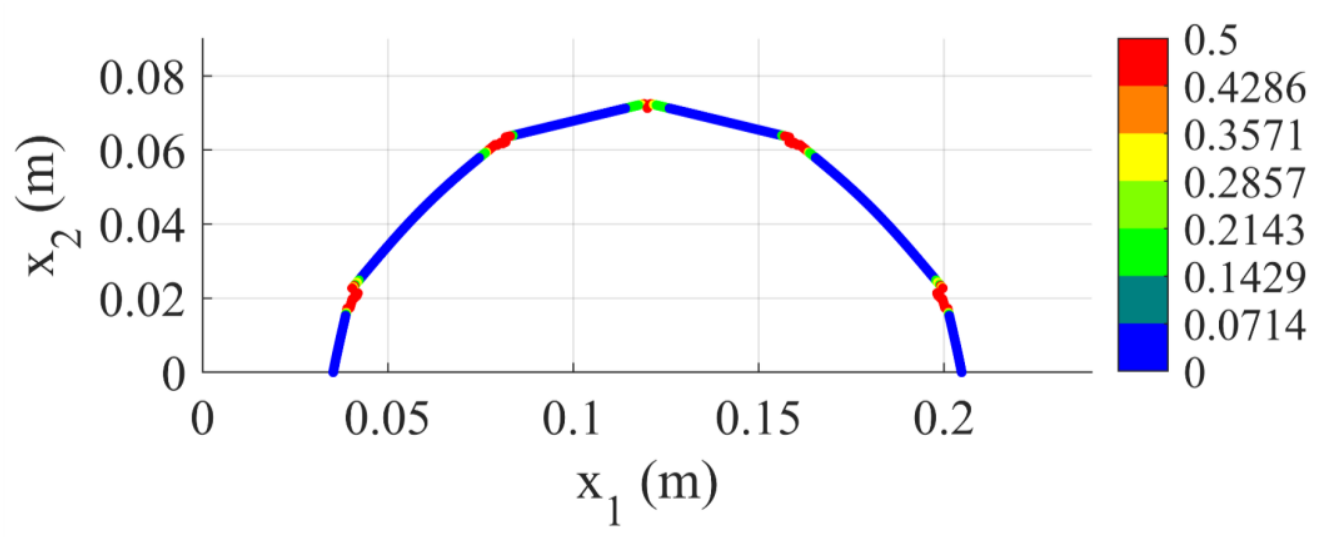

(c)

Fig. 19. Damage evolution on the dry spaghetti subjected to pure bending (a): first damage occurrence at (a) $178 \times 10^{5}$ (b): $178.26 \times 10^{5}$, (c): $178.62 \times 10^{5}$ time steps

\subsubsection{Case 2: spaghetti subjected to a combined loading: torsion and bending}

In this case, the boundary and loading conditions applied for the beam include three stages as follows. 
(1) Stage 1: the beam is twisted by $\theta^{*}=100^{\circ}\left(\theta_{1}(0,0,0)=-\theta^{*} / 2, \quad \theta_{1}(L, 0,0)=\theta^{*} / 2\right)$ as shown in Fig. 20.

(2) Stage 2: In addition to torsional loading, the beam is subjected to incremental bending moments at two ends as shown in Fig. 21. The loading conditions in this stage are given in Eq. (51).

(3) Stage 3: when the first damage occurs, the bending moments are released. The beam is fixed at two ends and the remaining parts of the beam can be freely moved as shown in Fig. 22. In all stages, the beam is subjected to the gravitational forces as given in Eq. (52).

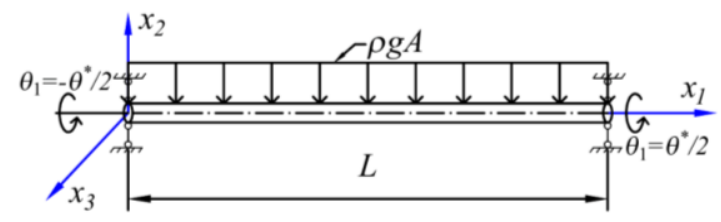

(a)

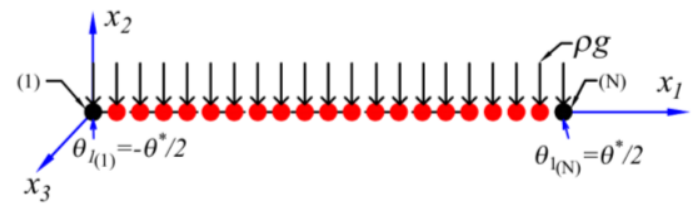

(b)

Fig. 20. Spaghetti subjected to torsion in stage 1 (a): geometry, (b): PD discretized model

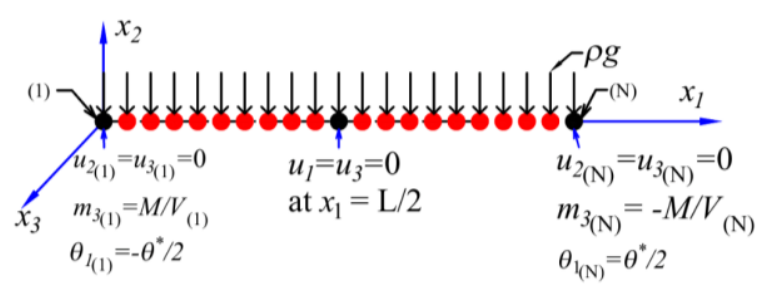

(a)

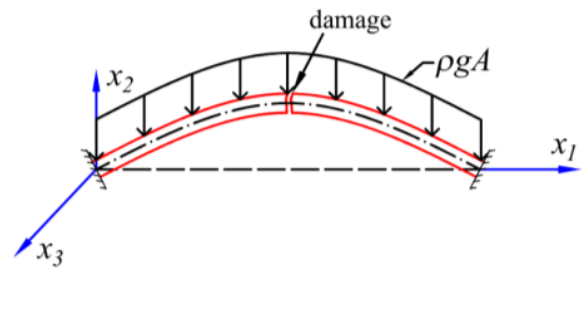

(b)

Fig. 21. Spaghetti subjected to torsion and bending in stage 2 (a): PD discretized model, (b): deformed configuration at the end of stage 2 when the first damage occurs

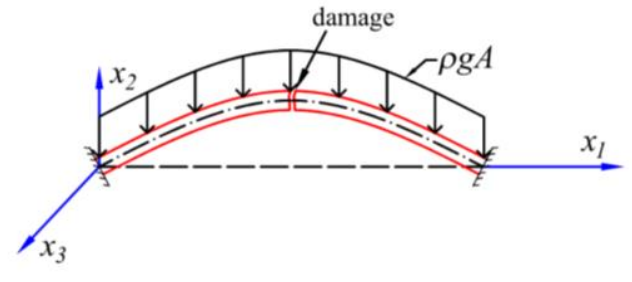

(a)

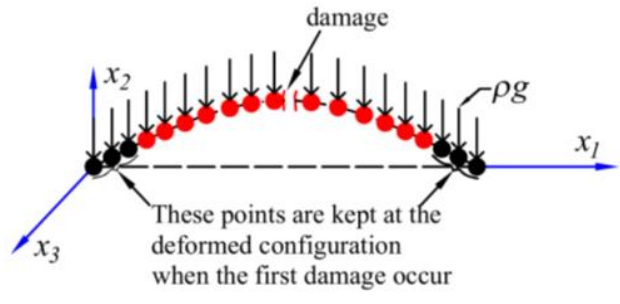

(b)

Fig. 22. Boundary and loading conditions for the spaghetti in stage 3 (a): deformed configuration when the first damage occur and the bending moments and torsional loading are released, (b):

PD discretized model

Similarly, the beam is considered in quasi-static loading conditions and the PD solution is obtained by using the ADR method. In stage 1 for the torsional loading, the converged solution is obtained after $N_{0}=2 \times 10^{5}$ time step. In the second stage, the torsional angles, $\theta_{1}$ of the material points located at two ends of the beam are kept constant, meanwhile, the incremental bending moments 
are applied on two ends of the beam as given in Eq. (51). Fig. 23 shows the damage evolution on the beam in the combined loading conditions. Similar to the previous loading condition in Section 7.2.1, the first damage occurs in the middle section of the beam at $N_{0}+175.6 \times 10^{5}$ load step as shown in Fig. 23(a). Later, two more new damage locations occur symmetrically with respect to the middle section of the beam as shown in Fig. 23(b). As can be observed from the figure, in this loading condition, the beam is only damaged into 4 pieces. This prediction also agrees with the experimental results in [76].

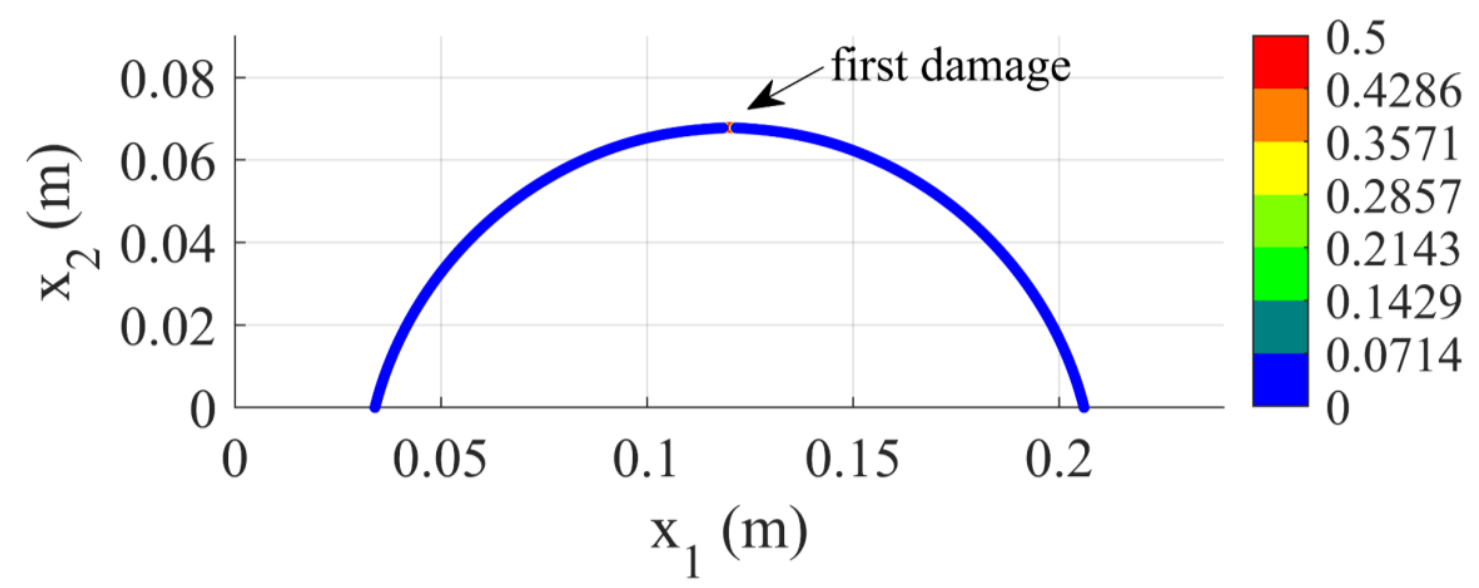

(a)

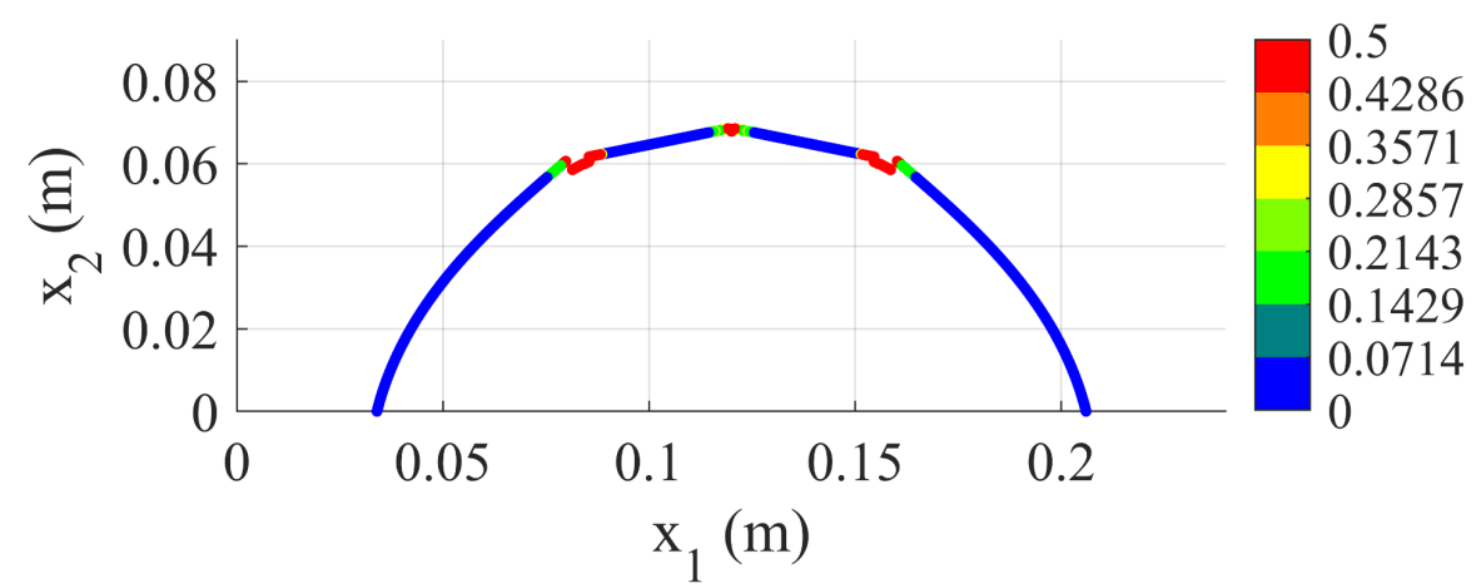

(b)

Fig. 23. Damage evolution on the dry spaghetti subjected to torsion and bending (a): first damage occurrence at (a) $N_{0}+175.6 \times 10^{5}$ time steps (when the first damage occurs), (b): $N_{0}+177.08 \times 10^{5}$ time steps. $\left(N_{0}=2 \times 10^{5}\right.$ time steps for obtaining a converged solution for torsional loading in stage 1) 


\section{Concluding remarks}

This study provides a novel nonlinear PD beam model with 6 degrees of freedom. The numerical procedure for investigating large deformations of beam structures is also introduced. The accuracy of the nonlinear PD model is verified for both straight and curved beams. The results from nonlinear PD analyses have good agreement with those in nonlinear FEA solutions, as well as the results from previous studies in the literature. For damage predictions, an energy-based damage criterion for the nonlinear beam model is proposed. The developed nonlinear PD model is used to predict damages on a spaghetti subjected to different loading conditions. The predicted results show good agreement with the experimental results given in the literature. The developed PD model can be used for any type of beam structure to predict possible damages that may occur during the operation process.

\section{Acknowledgment}

The authors gratefully acknowledge the support from the Ministry of Education and Training in Vietnam and the University of Strathclyde. Results were obtained using the ARCHIE-WeSt High Performance Computer (www.archie-west.ac.uk) based at the University of Strathclyde. 


\section{Appendix. A. Peridynamic constants}

In this section, the derivations to obtain the PD constants are presented. First, let $k$ and $j$ be two material points in a beam structure. By using Taylor's series expansion, a variable $f\left({ }^{0} x_{(j)}\right)$ at material point $j$ can be expressed in term of the variable $f\left({ }^{0} x_{(k)}\right)$ at material point $k$ as

$f\left({ }^{0} x_{(j)}\right)=f\left({ }^{0} x_{(k)}\right)+\left({ }^{0} x_{(j)}-{ }^{0} x_{(k)}\right) \frac{\partial}{\partial{ }^{0} x} f\left({ }^{0} x_{(k)}\right)+\cdots$

or

$f\left({ }^{0} x_{(j)}\right)=f\left({ }^{0} x_{(k)}\right)+{ }_{0} f_{, x(k)}\left({ }^{0} x_{(j)}-{ }^{0} x_{(k)}\right)+\cdots$

with

${ }_{0} f_{, x(k)}=\frac{\partial}{\partial^{0} x} f\left({ }^{0} x_{(k)}\right)$

In Eq. (A1), the parameter $f$ can be any variable. For instance, $f$ can be the displacements and rotations of material points, $\left(u, v, w, \theta_{x}, \theta_{y}, \theta_{z}\right)$.

If $k$ and $j$ are two different material points $(k \neq j)$, the distance between them in the undeformed configuration is nonzero $\left({ }^{0} \xi_{(k)(j)} \neq 0\right)$. Therefore, Eq. (A1b) can be rewritten as

$\frac{f\left({ }^{0} x_{(j)}\right)-f\left({ }^{0} x_{(k)}\right)}{{ }^{0} \xi_{(k)(j)}}={ }_{0} f_{, x(k)} \frac{{ }^{0} x_{(j)}-{ }^{0} x_{(k)}}{{ }^{0} \xi_{(k)(j)}}+\cdots$

By using the relations given in Eq. (29h), Eq. (A2) can be rewritten as

$\frac{f\left({ }^{0} x_{(j)}\right)-f\left({ }^{0} x_{(k)}\right)}{{ }^{0} \xi_{(k)(j)}}={ }_{0} f_{, x(k)}{ }^{0} \beta_{(k)(j)}+\cdots$

Replacing $f$ variable in Eq. (A3) by displacements, rotations, and director vectors at time $t$ of material points $k$ and $j$ results in the following relations

$$
\begin{aligned}
& \frac{{ }^{t} u_{(j)}-{ }^{t} u_{(k)}}{{ }^{0} \xi_{(k)(j)}}={ }_{0}^{t} u_{, x(k)} \beta_{(k)(j)}+\cdots \\
& \frac{{ }^{t} v_{(j)}-{ }^{t} v_{(k)}}{{ }^{0} \xi_{(k)(j)}}={ }_{0}^{t} v_{, x(k)}{ }^{0} \beta_{(k)(j)}+\cdots \\
& \frac{{ }^{t} w_{(j)}-{ }^{t} w_{(k)}}{{ }^{0} \xi_{(k)(j)}}={ }_{0}^{t} w_{, x(k)}{ }^{0} \beta_{(k)(j)}+\cdots \\
& \frac{{ }^{t} \theta_{x(j)}-{ }^{t} \theta_{x(k)}}{{ }^{0} \xi_{(k)(j)}}={ }_{0}^{t} \theta_{x, x(k)}{ }^{0} \beta_{(k)(j)}+\cdots \\
& \frac{{ }^{t} \theta_{y(j)}-{ }^{t} \theta_{y(k)}}{{ }^{0} \xi_{(k)(j)}}={ }_{0}^{t} \theta_{y, x(k)} \beta_{(k)(j)}+\cdots \\
& \frac{{ }^{t} \theta_{z(j)}-{ }^{t} \theta_{z(k)}}{{ }^{0} \xi_{(k)(j)}}={ }_{0}^{t} \theta_{z, x(k)}{ }^{0} \beta_{(k)(j)}+\cdots
\end{aligned}
$$




$$
\begin{aligned}
& \frac{{ }^{t} V_{s x(j)}-{ }^{t} V_{s x(k)}}{{ }^{0} \xi_{(k)(j)}}={ }_{0}^{t} V_{s x, x(k)}{ }^{0} \beta_{(k)(j)}+\cdots \\
& \frac{{ }^{t} V_{s y(j)}-{ }^{t} V_{s y(k)}}{{ }^{0} \xi_{(k)(j)}}={ }_{0}^{t} V_{s y, x(k)}{ }^{0} \beta_{(k)(j)}+\cdots \\
& \frac{{ }^{t} V_{s z(j)}-{ }^{t} V_{s z(k)}}{{ }^{0} \xi_{(k)(j)}}={ }_{0}^{t} V_{s z, x(k)}{ }^{0} \beta_{(k)(j)}+\cdots \\
& \frac{{ }^{t} V_{t x(j)}-{ }^{t} V_{t x(k)}}{{ }^{0} \xi_{(k)(j)}}={ }_{0}^{t} V_{t x, x(k)}{ }^{0} \beta_{(k)(j)}+\cdots \\
& \frac{{ }^{t} V_{t y(j)}-{ }^{t} V_{t y(k)}}{{ }^{0} \xi_{(k)(j)}}={ }_{0}^{t} V_{t y, x(k)}{ }^{0} \beta_{(k)(j)}+\cdots \\
& \frac{{ }^{t} V_{t z(j)}-{ }^{t} V_{t z(k)}}{{ }^{0} \xi_{(k)(j)}}={ }_{0}^{t} V_{t z, x(k)}{ }^{0} \beta_{(k)(j)}+\cdots
\end{aligned}
$$

Similarly, replacing $f$ in Eq. (A3) by incremental displacements and incremental rotations (from time $t$ to time $t+\Delta t$ ) of material points $k$ and $j$ results in the following relations

$$
\begin{aligned}
& \frac{\underline{u}_{(j)}-\underline{u}_{(k)}}{{ }^{0} \xi_{(k)(j)}}={ }_{0} \underline{u}_{, x(k)}{ }^{0} \beta_{(k)(j)}+\cdots \\
& \frac{\underline{v}_{(j)}-\underline{v}_{(k)}}{{ }^{0} \xi_{(k)(j)}}={ }_{0} \underline{v}_{, x(k)}{ }^{0} \beta_{(k)(j)}+\cdots \\
& \frac{\underline{w}_{(j)}-\underline{w}_{(k)}}{{ }^{0} \xi_{(k)(j)}}={ }_{0} \underline{w}_{, x(k)}{ }^{0} \beta_{(k)(j)}+\cdots \\
& \frac{\underline{\theta}_{x(j)}-\underline{\theta}_{x(k)}}{{ }^{0} \xi_{(k)(j)}}={ }_{0} \underline{\theta}_{x, x(k)}{ }^{0} \beta_{(k)(j)}+\cdots \\
& \frac{\underline{\theta}_{y(j)}-\underline{\theta}_{y(k)}}{{ }^{0} \xi_{(k)(j)}}={ }_{0} \underline{\theta}_{y, x(k)}{ }^{0} \beta_{(k)(j)}+\cdots \\
& \frac{\underline{\theta}_{z(j)}-\underline{\theta}_{z(k)}}{{ }^{0} \xi_{(k)(j)}}={ }_{0} \underline{\theta}_{z, x(k)}{ }^{0} \beta_{(k)(j)}+\cdots
\end{aligned}
$$

Therefore, by using the relation given in Eq. (A4) and Eq. (A5), the terms, ${ }_{0}^{t} a_{1}^{P D},{ }_{0}^{t} a_{2}^{P D},{ }_{0}^{t} a_{3}^{P D}$ given in Eq. (29d-f) can be rewritten as

$$
\begin{aligned}
& { }_{0}^{t} a_{1}^{P D}={ }_{0}^{t} u_{, x(k)}\left({ }^{0} \beta_{(k)(j)}\right)^{2}={ }_{0}^{t} u_{, x(k)} \\
& { }_{0}^{t} a_{2}^{P D}={ }_{0}^{t} v_{, x(k)}\left({ }^{0} \beta_{(k)(j)}\right)^{2}={ }_{0}^{t} v_{, x(k)}
\end{aligned}
$$


${ }_{0}^{t} a_{3}^{P D}={ }_{0}^{t} w_{, x(k)}\left({ }^{0} \beta_{(k)(j)}\right)^{2}={ }_{0}^{t} w_{, x(k)}$

with

$\left({ }^{0} \beta_{(k)(j)}\right)^{2}=\left(\frac{{ }^{0} x_{(j)}-{ }^{0} x_{(k)}}{{ }^{0} \xi_{(k)(j)}}\right)^{2}=1$

Similarly, by using the relation given in Eq. (A4) and Eq. (A5), the terms, ${ }_{0}^{t} a_{4}^{P D},{ }_{0}^{t} a_{5}^{P D}, \ldots,{ }_{0}^{t} a_{9}^{P D}$ given in Eq. (31f-k) can be rewritten as

${ }_{0}^{t} a_{4}^{P D}={ }_{0}^{t} V_{s x, x(k)}$

${ }_{0}^{t} a_{5}^{P D}={ }_{0}^{t} V_{s y, x(k)}$

${ }_{0}^{t} a_{6}^{P D}={ }_{0}^{t} V_{s z, x(k)}$

${ }_{0}^{t} a_{7}^{P D}={ }_{0}^{t} V_{t x, x(k)}$

${ }_{0}^{t} a_{8}^{P D}={ }_{0}^{t} V_{t y, x(k)}$

${ }_{0}^{t} a_{9}^{P D}={ }_{0}^{t} V_{t z, x(k)}$

Therefore, the terms, ${ }_{0}^{t} a_{10}^{P D},{ }_{0}^{t} a_{11}^{P D},{ }_{0}^{t} a_{12}^{P D}$ given in Eq. (29b) and Eq. (31b-c) can be obtained as

${ }_{0}^{t} a_{10}^{P D}={ }_{0}^{t} a_{1}^{P D}+\frac{1}{2}\left(\left({ }_{0}^{t} a_{1}^{P D}\right)^{2}+\left({ }_{0}^{t} a_{2}^{P D}\right)^{2}+\left({ }_{0}^{t} a_{3}^{P D}\right)^{2}\right)$

${ }_{0}^{t} a_{11}^{P D}={ }_{0}^{t} a_{4}^{P D}\left(1+{ }_{0}^{t} a_{1}^{P D}\right)+\left({ }_{0}^{t} a_{2}^{P D}\right)\left({ }_{0}^{t} a_{5}^{P D}\right)+\left({ }_{0}^{t} a_{3}^{P D}\right)\left({ }_{0}^{t} a_{6}^{P D}\right)$

${ }_{0}^{t} a_{12}^{P D}=\left({ }_{0}^{t} a_{7}^{P D}\right)\left(1+{ }_{0}^{t} a_{1}^{P D}\right)+\left({ }_{0}^{t} a_{2}^{P D}\right)\left({ }_{0}^{t} a_{8}^{P D}\right)+\left({ }_{0}^{t} a_{3}^{P D}\right)\left({ }_{0}^{t} a_{9}^{P D}\right)$

Moreover, by assuming that the beam is discretized with fine mesh, material points $k$ and $j$ are very close to each other within the horizon size. Therefore, the following approximation can be assumed

$$
\begin{array}{lll}
\frac{{ }^{t} V_{s x(j)}+{ }^{t} V_{s x(k)}}{2} \approx V_{s x(k)} ; & \frac{{ }^{t} V_{s y(j)}+{ }^{t} V_{s y(k)}}{2} \approx{ }^{t} V_{s y(k)} ; & \frac{{ }^{t} V_{s z(j)}+{ }^{t} V_{s z(k)}}{2} \approx{ }^{t} V_{s z(k)} \\
\frac{{ }^{t} V_{t x(j)}+{ }^{t} V_{t x(k)}}{2} \approx{ }^{t} V_{t x(k)} ; & \frac{{ }^{t} V_{t y(j)}+{ }^{t} V_{t y(k)}}{2} \approx{ }^{t} V_{t y(k)} ; & \frac{{ }^{t} V_{t z(j)}+{ }^{t} V_{t z(k)}}{2} \approx{ }^{t} V_{t z(k)}
\end{array}
$$

Therefore, by using the relations given in Eq. (A9), the terms, ${ }_{0}^{t} a_{13}^{P D},{ }_{0}^{t} a_{14}^{P D}$ and ${ }_{0}^{t} a_{15}^{P D}$ given in Eq. (33b-c) and Eq. (35b) can be approximated as

$$
\begin{aligned}
& { }_{0}^{t} a_{13}^{P D}=\left(1+{ }_{0}^{t} a_{1}^{P D}\right)^{t} V_{s x(k)}+\left({ }_{0}^{t} a_{2}^{P D}\right)^{t} V_{s y(k)}+\left({ }_{0}^{t} a_{3}^{P D}\right)^{t} V_{s z(k)} \\
& { }_{0}^{t} a_{14}^{P D}=\left(1+{ }_{0}^{t} a_{1}^{P D}\right)^{t} V_{t x(k)}+\left({ }_{0}^{t} a_{2}^{P D}\right)^{t} V_{t y(k)}+\left({ }_{0}^{t} a_{3}^{P D}\right)^{t} V_{t z(k)}
\end{aligned}
$$


${ }_{0}^{t} a_{15}^{P D}=\left({ }_{0}^{t} a_{8}^{P D}\right)^{t} V_{s y(k)}+\left({ }_{0}^{t} a_{7}^{P D}\right)^{t} V_{s x(k)}+\left({ }_{0}^{t} a_{9}^{P D}\right)^{t} V_{s z(k)}$

By comparing Eq. (A6-8), Eq. (A10) to Eq. (22e-m), it can be observed that the terms, ${ }_{0}^{t} a_{1}^{P D}, \ldots,{ }_{0}^{t} a_{15}^{P D}$ in PD and the terms ${ }_{0}^{t} a_{1}, \ldots,{ }_{0}^{t} a_{15}$ in classical continuum mechanics are equal to each other.

Similarly, by using the relations given in Eq. (A4-5), the terms ${ }_{0} \underline{a}_{10}^{P D},{ }_{0} \underline{a}_{11}^{P D},{ }_{0} \underline{a}_{12}^{P D}$ given in Eq. (29c) and Eq. (31d-e) can be rewritten as

$$
\begin{aligned}
& { }_{0} \underline{a}_{10}^{P D}=\left({ }_{0} \underline{u}_{, x(k)}\right)\left(1+{ }_{0}^{t} a_{1}^{P D}\right)+{ }_{0}^{t} a_{2}^{P D}\left({ }_{0} \underline{\underline{v}}_{, x(k)}\right)+{ }_{0}^{t} a_{3}^{P D}\left({ }_{0} \underline{w}_{, x(k)}\right) \\
& { }_{0} \underline{\underline{a}}_{11}^{P D}=\left(\begin{array}{l}
\left({ }_{0}^{t} a_{4}^{P D}\right)\left({ }_{0} \underline{u}_{, x(k)}\right)+\left({ }_{0}^{t} a_{5}^{P D}\right)\left({ }_{0} \underline{\underline{v}}_{, x(k)}\right)+\left({ }_{0}^{t} a_{6}^{P D}\right)\left({ }_{0} \underline{w}_{, x(k)}\right) \\
+\left(1+{ }_{0}^{t} a_{1}^{P D}\right) \frac{\partial}{\partial^{0} x}\left(\underline{\theta}_{y(k)}{ }^{t} V_{s z(k)}-\underline{\theta}_{z(k)}{ }^{t} V_{s y(k)}\right)+\left({ }_{0}^{t} a_{2}^{P D}\right) \frac{\partial}{\partial^{0} x}\left(\underline{\theta}_{z(k)}{ }^{t} V_{s x(k)}-\underline{\theta}_{x(k)}{ }^{t} V_{s z(k)}\right) \\
+\left({ }_{0}^{t} a_{3}^{P D}\right) \frac{\partial}{\partial^{0} x}\left(\underline{\theta}_{x(k)}{ }^{t} V_{s y(k)}-\underline{\theta}_{y(k)}{ }^{t} V_{s x(k)}\right)
\end{array}\right) \\
& { }_{0} \underline{a}_{12}^{P D}=\left(\begin{array}{l}
\left({ }_{0}^{t} a_{7}^{P D}\right)\left({ }_{0} \underline{u}_{, x(k)}\right)+\left({ }_{0}^{t} a_{8}^{P D}\right)\left({ }_{0} \underline{\underline{v}}_{, x(k)}\right)+\left({ }_{0}^{t} a_{9}^{P D}\right)\left({ }_{0} \underline{w}_{, x(k)}\right) \\
+\left(1+{ }_{0}^{t} a_{1}^{P D}\right) \frac{\partial}{\partial^{0} x}\left(\underline{\theta}_{y(k)}{ }^{t} V_{t z(k)}-\underline{\theta}_{z(k)}{ }^{t} V_{t y(k)}\right)+\left({ }_{0}^{t} a_{2}^{P D}\right) \frac{\partial}{\partial^{0} x}\left(\underline{\theta}_{z(k)}{ }^{t} V_{t x(k)}-\underline{\theta}_{x(k)}{ }^{t} V_{t z(k)}\right) \\
+\left({ }_{0}^{t} a_{3}^{P D}\right) \frac{\partial}{\partial^{0} x}\left(\underline{\theta}_{x(k)}{ }^{t} V_{t y(k)}-\underline{\theta}_{y(k)}{ }^{t} V_{t x(k)}\right)
\end{array}\right)
\end{aligned}
$$

By using the relations given in Eq. (A4-5) and Eq. (A9), the terms ${ }_{0} \underline{a}_{13}^{P D},{ }_{0} \underline{a}_{14}^{P D}$ and ${ }_{0} \underline{a}_{15}^{P D}$ given in Eq. (33d-e) and Eq. (35c) can be rewritten as

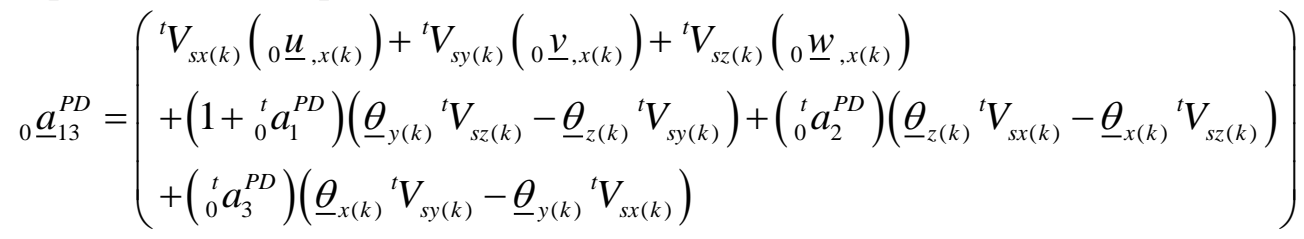

$$
\begin{aligned}
& { }_{0} \underline{a}_{14}^{P D}=\left(\begin{array}{l}
{ }^{t} V_{t x(k)}\left({ }_{0} \underline{u}_{, x(k)}\right)+{ }^{t} V_{t y(k)}\left({ }_{0} \underline{\underline{v}}_{, x(k)}\right)+{ }^{t} V_{t z(k)}\left({ }_{0} \underline{w}_{, x(k)}\right) \\
+\left(1+{ }_{0}^{t} a_{1}^{P D}\right)\left(\underline{\theta}_{y(k)}{ }^{t} V_{t z(k)}-\underline{\theta}_{z(k)}{ }^{t} V_{t y(k)}\right)+\left({ }_{0}^{t} a_{2}^{P D}\right)\left(\underline{\theta}_{z(k)}{ }^{t} V_{t x(k)}-\underline{\theta}_{x(k)}{ }^{t} V_{t z(k)}\right) \\
+\left({ }_{0}^{t} a_{3}^{P D}\right)\left(\underline{\theta}_{x(k)}{ }^{t} V_{t y(k)}-\underline{\theta}_{y(k)}{ }^{t} V_{t x(k)}\right)
\end{array}\right) \\
& { }_{0} \underline{a}_{15}^{P D}=\left(\begin{array}{l}
\left({ }^{t} V_{t y(k)}{ }^{t} V_{s z(k)}-{ }^{t} V_{t z(k)}{ }^{t} V_{s y(k)}\right)\left({ }_{0} \underline{\theta}_{x, x(k)}\right)+\left({ }^{t} V_{t z(k)}{ }^{t} V_{s x(k)}-{ }^{t} V_{t x(k)}{ }^{t} V_{s z(k)}\right)\left({ }_{0} \underline{\theta}_{y, x(k)}\right) \\
+\left({ }^{t} V_{t x(k)}{ }^{t} V_{s y(k)}-{ }^{t} V_{t y(k)}{ }^{t} V_{s x(k)}\right)\left({ }_{0} \underline{\theta}_{z, x(k)}\right)
\end{array}\right)
\end{aligned}
$$

By comparing Eq. (A11-12) with Eq. (22n-s), it can be observed that the terms, ${ }_{0} \underline{a}_{10}^{P D},{ }_{0} \underline{a}_{11}^{P D}, \ldots,{ }_{0} \underline{a}_{15}^{P D}$ in PD and the terms ${ }_{0} \underline{a}_{10},{ }_{0} \underline{a}_{11}, \ldots,{ }_{0} \underline{a}_{15}$ in classical continuum mechanics are equal to each other. 


\section{A.1. Peridynamic constant for axial deformations}

By disregarding the peridynamic interactions beyond the horizon, the PD strain energy density for axial deformations given in Eq. (29a) can be rewritten in integral form as

$\bar{W}_{\text {axial }}^{\text {NLPD }}=\left({ }^{0} A\right) \int_{0}^{\delta} C_{a x}\left({ }_{0}^{t} a_{10}^{P D}\right)\left({ }_{0} \underline{a}_{10}^{P D}\right)\left({ }^{0} \xi_{(k)(j)}\right) d\left({ }^{0} \xi_{(k)(j)}\right)$

By performing the integrations in Eq. (A13), the nonlinear PD strain energy density for axial deformations can be rewritten as

$\bar{W}_{\text {axial }}^{\text {NLPD }}=\frac{1}{2}\left({ }^{0} A\right) \delta^{2} C_{a x}\left({ }_{0}^{t} a_{10}^{P D}\right)\left({ }_{0} \underline{a}_{10}^{P D}\right)$

As proved in the previous section, the terms ${ }_{0}^{t} a_{10}^{P D},{ }_{0} \underline{a}_{10}^{P D}$ in PD and ${ }_{0}^{t} a_{10},{ }_{0} \underline{a}_{10}$ in classical continuum mechanics are equal to each other. Therefore, by comparing Eq. (29a) and Eq. (A14), the PD bond constant for axial deformations, $C_{a x}$ can be obtained as

$$
C_{a x}=\frac{2 E}{\left({ }^{0} A\right) \delta^{2}}
$$

\section{A.2. PD constants for bending deformations}

By disregarding interactions beyond the horizon of material point $k$, the PD strain energy density for bending deformations given in Eq. (31a) can be rewritten as

$$
\begin{aligned}
\bar{W}_{\text {bending }}^{\text {NLPD }} & =C_{b z}\left({ }^{0} A\right) \int_{0}^{\delta}\left({ }_{0}^{t} a_{11}^{P D}\right)\left({ }_{0} \underline{a}_{11}^{P D}\right)\left({ }^{0} \xi_{(k)(j)}\right) d\left({ }^{0} \xi_{(k)(j)}\right) \\
& +C_{b y}\left({ }^{0} A\right) \int_{0}^{\delta}\left({ }_{0}^{t} a_{12}^{P D}\right)\left({ }_{0} \underline{P}_{12}^{P D}\right)\left({ }^{0} \xi_{(k)(j)}\right) d\left({ }^{0} \xi_{(k)(j)}\right)
\end{aligned}
$$

By performing the integrations in Eq. (A16), the SED for bending deformations can be rewritten as

$$
\bar{W}_{\text {bending }}^{\text {NLPD }}=\frac{1}{2} C_{b z}\left({ }^{0} A\right) \delta^{2}\left({ }_{0}^{t} a_{11}^{P D}\right)\left({ }_{0} \underline{a}_{11}^{P D}\right)+\frac{1}{2} C_{b y}\left({ }^{0} A\right) \delta^{2}\left({ }_{0}^{t} a_{12}^{P D}\right)\left({ }_{0} \underline{a}_{12}^{P D}\right)
$$

As proved in the previous section, the terms ${ }_{0}^{t} a_{11}^{P D},{ }_{0} \underline{a}_{11}^{P D},{ }_{0}^{t} a_{12}^{P D},{ }_{0} \underline{a}_{12}^{P D}$ in PD and ${ }_{0}^{t} a_{11},{ }_{0} \underline{a}_{11},{ }_{0}^{t} a_{12}$, ${ }_{0} \underline{a}_{12}$ in classical continuum mechanics are equal to each other. Therefore, by comparing Eq. (22b) and Eq. (A17), the PD bond constants for bending deformations, $C_{b y}$ and $C_{b z}$, can be represented as

$$
\begin{aligned}
C_{b y} & =\frac{2 E I_{y y}}{\left({ }^{0} A\right)^{2} \delta^{2}} \\
C_{b z} & =\frac{2 E I_{z z}}{\left({ }^{0} A\right)^{2} \delta^{2}}
\end{aligned}
$$

\section{A.3. PD constants for shear deformations}

By disregarding interactions beyond the horizon of material point $k$, the PD strain energy density for bending deformations given in Eq. (33a) can be rewritten as 
$\bar{W}_{\text {shear }}^{N L P D}=C_{\text {sh }}\left({ }^{0} A\right) \int_{0}^{\delta}\left[\left({ }_{0}^{t} a_{13}^{P D}\right)\left({ }_{0} \underline{a}_{13}^{P D}\right)+\left({ }_{0}^{t} a_{14}^{P D}\right)\left({ }_{0} \underline{a}_{14}^{P D}\right)\right]\left({ }^{0} \xi_{(k)(j)}\right) d\left({ }^{0} \xi_{(k)(j)}\right)$

By performing the integrations in Eq. (A19), the SED for shear deformations can be rewritten as

$\bar{W}_{\text {shear }}^{N L P D}=\frac{1}{2} C_{s h}\left({ }^{0} A\right) \delta^{2}\left[\left({ }_{0}^{t} a_{13}^{P D}\right)\left({ }_{0} \underline{a}_{13}^{P D}\right)+\left({ }_{0}^{t} a_{14}^{P D}\right)\left({ }_{0} \underline{a}_{14}^{P D}\right)\right]$

As proved in the previous section, the terms ${ }_{0}^{t} a_{13}^{P D},{ }_{0} \underline{a}_{13}^{P D},{ }_{0}^{t} a_{14}^{P D},{ }_{0} \underline{a}_{14}^{P D}$ in PD and ${ }_{0}^{t} a_{13},{ }_{0} \underline{a}_{13},{ }_{0}^{t} a_{14}$, ${ }_{0} \underline{a}_{14}$ in classical continuum mechanics are equal to each other. Therefore, by comparing Eq. (22c) and Eq. (A20), the PD bond constants for shear deformations, $C_{s h}$, can be represented as

$$
C_{s h}=\frac{2 k_{s} G}{\left({ }^{0} A\right) \delta^{2}}
$$

\section{A.4. PD constants for torsional deformations}

By disregarding interactions beyond the horizon of material point $k$, the PD strain energy density for bending deformations given in Eq. (35a) can be rewritten as

$\bar{W}_{\text {torsion }}^{N L P D}=C_{t}\left({ }^{0} A\right) \int_{0}^{\delta}\left({ }_{0}^{t} a_{15}^{P D}\right)\left({ }_{0} \underline{a}_{15}^{P D}\right)\left({ }^{0} \xi_{(k)(j)}\right) d\left({ }^{0} \xi_{(k)(j)}\right)$

By performing the integrations in Eq. (A22), the nonlinear PD strain energy density for torsional deformations can be rewritten as

$\bar{W}_{\text {torsion }}^{N L P D}=\frac{1}{2} C_{t}\left({ }^{0} A\right) \delta^{2}\left({ }_{0}^{t} a_{15}^{P D}\right)\left({ }_{0} \underline{a}_{15}^{P D}\right)$

As proved in the previous section, the terms ${ }_{0}^{t} a_{15}^{P D},{ }_{0} \underline{a}_{15}^{P D}$ in PD and ${ }_{0}^{t} a_{15},{ }_{0} \underline{a}_{15}$ in classical continuum mechanics are equal to each other. Therefore, by comparing Eq. (22d) and Eq. (A23), the PD bond constants for torsional deformations, $C_{t}$ can be obtained as

$$
C_{t}=\frac{2 k_{t} G}{\left({ }^{0} A\right)^{2} \delta^{2}}
$$

\section{Appendix. B. Adaptive relaxation method}

As presented in Section 6 and Section 7, for static and quasi-static problems, the adaptive dynamic relaxation method can be used in PD analysis [67]. Specifically, the mass matrix $\mathbf{m}_{(k)}^{G}$ can be replaced by a mass stable vector $\mathbf{M}_{(k)}^{G}$, and a damping matrix $\mathbf{C}_{(k)}^{G}$ is added into the equation of motion given in Eq. (51b) as

$$
\mathbf{M}_{(k)}^{G}\left({ }^{t} \ddot{\mathbf{u}}_{(k)}^{G}\right)+\mathbf{C}_{(k)}^{G}\left({ }^{t} \dot{\mathbf{u}}_{(k)}^{G}\right)=\sum_{j=1}^{N} \psi_{(k)(j)}\left({ }_{0}^{t} \mathbf{f}_{(k)(j)}^{G}\right) v_{(k)(j)} \Omega_{(k)(j)}{ }^{0} V_{(j)}+{ }^{t} \mathbf{b}_{(k)}^{G}
$$

where ${ }^{t} \dot{\mathbf{u}}_{(k)}^{G}$ represents the velocity vector at time $t$.

As proposed by Nguyen and Oterkus [66], the mass stable vector in global coordinates can be presented as 
$\mathbf{M}_{(k)}^{G}=\left[\begin{array}{cccccc}M_{(k)}^{u_{1}} & 0 & 0 & 0 & 0 & 0 \\ 0 & M_{(k)}^{u_{2}} & 0 & 0 & 0 & 0 \\ 0 & 0 & M_{(k)}^{u_{3}} & 0 & 0 & 0 \\ 0 & 0 & 0 & M_{(k)}^{\theta_{1}} & 0 & 0 \\ 0 & 0 & 0 & 0 & M_{(k)}^{\theta_{2}} & 0 \\ 0 & 0 & 0 & 0 & 0 & M_{(k)}^{\theta_{3}}\end{array}\right]$

with

$M_{(k)}^{u_{1}}=M_{(k)}^{u_{2}}=M_{(k)}^{u_{3}}=\max \left(M_{(k)}^{u}, M_{(k)}^{v}, M_{(k)}^{w}\right)$

$M_{(k)}^{\theta_{1}}=M_{(k)}^{\theta_{2}}=M_{(k)}^{\theta_{3}}=\max \left(M_{(k)}^{\theta_{x}}, M_{(k)}^{\theta_{y}}, M_{(k)}^{\theta_{z}}\right)$

where $M_{(k)}^{u_{1}}, M_{(k)}^{u_{2}}, M_{(k)}^{u_{3}}, M_{(k)}^{\theta_{1}}, M_{(k)}^{\theta_{2}}, M_{(k)}^{\theta_{3}}$ represent the components mass stable vector corresponding to translational and rotational DOFs in the global coordinates. The terms, $M_{(k)}^{u}$, $M_{(k)}^{v}, M_{(k)}^{w}, M_{(k)}^{\theta_{x}}, M_{(k)}^{\theta_{y}}, M_{(k)}^{\theta_{z}}$ represent the components of a mass stable vector in the local coordinates. The components of the mass stable vector in the local coordinates can be calculated based on PD bond constants as $[15,78]$

$$
\begin{aligned}
M_{(k)}^{u} & \geq \frac{1}{4} \frac{d t^{2}\left({ }^{0} A\right) \delta}{\Delta x} C_{a x} \\
M_{(k)}^{v} & =M_{(k)}^{w} \geq \frac{1}{4} \frac{d t^{2}\left({ }^{0} A\right) \delta}{\Delta x} C_{s h} \\
M_{(k)}^{\theta_{x}} & \geq \frac{1}{4} \frac{d t^{2}\left({ }^{0} A\right) \delta}{\Delta x} C_{t} \\
M_{(k)}^{\theta_{y}} & \geq \frac{1}{4} \frac{d t^{2}\left({ }^{0} A\right) \delta}{\Delta x} C_{b y} \\
M_{(k)}^{\theta_{z}} & \geq \frac{1}{4} \frac{d t^{2}\left({ }^{0} A\right) \delta}{\Delta x} C_{b z}
\end{aligned}
$$

where $d t=1$ represents the time step for a quasi-static solution [73]. 


\section{References}

[1] Mindlin RD. Influence of rotatory inertia and shear on flexural motions of isotropic, elastic plates. J appl Mech 1951;18: 31-38.

[2] Reissner E. The effect of transverse shear deformation on the bending of elastic plates. J appl Mech 1945: A69-A77.

[3] Kanok-nukulchai W. A simple and efficient finite element for general shell analysis. International Journal for Numerical Methods in Engineering 1979;14(2): 179-200.

[4] Bathe K-j, Bolourchi S. A geometric and material nonlinear plate and shell element. Computers \& Structures 1980;11(1-2): 23-48.

[5] Pica A, Wood R, Hinton E. Finite element analysis of geometrically nonlinear plate behaviour using a Mindlin formulation. J Computers \& Structures 1980;11(3): 203-15.

[6] Cook R. Four-node 'flat'shell element: drilling degrees of freedom, membrane-bending coupling, warped geometry, and behavior. Computers \& structures 1994;50(4): 549-55.

[7] Bathe K-J. Finite element procedures: Klaus-Jurgen Bathe; 2006.

[8] Sussman T, Bathe K-J. 3D-shell elements for structures in large strains. Computers \& structures 2013;122: 2-12.

[9] Bauchau O, Craig J. Euler-Bernoulli beam theory. Structural analysis: Springer; 2009. 173221.

[10] Bathe KJ, Cimento AP. Some practical procedures for the solution of nonlinear finite element equations. Computer methods in applied mechanics engineering 1980;22(1): 59-85. [11] Silling SA. Reformulation of elasticity theory for discontinuities and long-range forces. Journal of the Mechanics and Physics of Solids 2000;48(1): 175-209.

[12] Silling SA, Askari E. A meshfree method based on the peridynamic model of solid mechanics. Computers \& structures 2005;83(17-18): 1526-35.

[13] Silling SA, Epton M, Weckner O, Xu J, Askari E. Peridynamic states and constitutive modeling. Journal of Elasticity 2007;88(2): 151-84.

[14] Silling SA, Lehoucq R. Peridynamic theory of solid mechanics. Advances in applied mechanics: Elsevier; 2010. 73-168.

[15] Madenci E, Oterkus E. Peridynamic Theory and Its Applications: Springer; 2014. [16] Ha YD, Bobaru F. Studies of dynamic crack propagation and crack branching with peridynamics. International Journal of Fracture 2010;162(1-2): 229-44.

[17] Bobaru F, Hu W. The meaning, selection, and use of the peridynamic horizon and its relation to crack branching in brittle materials. International journal of fracture 2012;176(2):

215-22.

[18] Agwai A, Guven I, Madenci E. Predicting crack propagation with peridynamics: a comparative study. International journal of fracture 2011;171(1): 65 .

[19] Kilic B, Madenci E. Prediction of crack paths in a quenched glass plate by using peridynamic theory. International journal of fracture 2009;156(2): 165-77.

[20] Ha YD, Bobaru F. Characteristics of dynamic brittle fracture captured with peridynamics. Engineering Fracture Mechanics 2011;78(6): 1156-68.

[21] Nguyen CT, Oterkus S. Ordinary state-based peridynamic model for geometrically nonlinear analysis. Engineering Fracture Mechanics 2019.

[22] Foster JT, Silling SA, Chen WW. Viscoplasticity using peridynamics. International journal for numerical methods in engineering 2010;81(10): 1242-58. 
[23] Madenci E, Oterkus S. Ordinary state-based peridynamics for plastic deformation according to von Mises yield criteria with isotropic hardening. Journal of the Mechanics Physics of Solids 2016;86: 192-219.

[24] Huang Y, Oterkus S, Hou H, Oterkus E, Wei Z, Zhang S. Peridynamic model for viscohyperelastic material deformation in different strain rates. Continuum Mechanics Thermodynamics 2019: 1-35.

[25] Madenci E, Oterkus S. Ordinary state-based peridynamics for thermoviscoelastic deformation. Engineering Fracture Mechanics 2017;175: 31-45.

[26] Mitchell JA. A non-local, ordinary-state-based viscoelasticity model for peridynamics. Sandia National Lab Report 2011;8064: 1-28.

[27] Oterkus S, Madenci E, Agwai A. Fully coupled peridynamic thermomechanics. Journal of the Mechanics Physics of Solids 2014;64: 1-23.

[28] Oterkus S, Madenci E, Oterkus E. Fully coupled poroelastic peridynamic formulation for fluid-filled fractures. Engineering geology 2017;225: 19-28.

[29] Gao Y, Oterkus S. Fully coupled thermomechanical analysis of laminated composites by using ordinary state based peridynamic theory. Composite Structures 2019;207: 397-424.

[30] Gao Y, Oterkus S. Non-local modeling for fluid flow coupled with heat transfer by using peridynamic differential operator. Engineering Analysis with Boundary Elements 2019;105: 10421.

[31] Diyaroglu C, Oterkus S, Oterkus E, Madenci E. Peridynamic modeling of diffusion by using finite-element analysis. IEEE Transactions on Components, Packaging Manufacturing Technology 2017;7(11): 1823-31.

[32] Wang H, Oterkus E, Oterkus S. Peridynamic modelling of fracture in marine lithium-ion batteries. Ocean Engineering 2018;151: 257-67.

[33] Madenci E, Oterkus S. Peridynamics for coupled field equations. Handbook of Peridynamic Modeling 2016: 489-531.

[34] Oterkus S. Peridynamics for the solution of multiphysics problems: The University of Arizona; 2015.

[35] Nguyen CT, Oterkus S. Peridynamics for the thermomechanical behavior of shell structures. Engineering Fracture Mechanics 2019: 106623.

[36] Wang H, Oterkus E, Oterkus S. Predicting fracture evolution during lithiation process using peridynamics. Engineering Fracture Mechanics 2018;192: 176-91.

[37] Madenci E, Oterkus S. Peridynamic modeling of thermo-oxidative damage evolution in a composite lamina. 58th AIAA/ASCE/AHS/ASC Structures, Structural Dynamics, and Materials Conference2017. 0197.

[38] Amani J, Oterkus E, Areias P, Zi G, Nguyen-Thoi T, Rabczuk T. A non-ordinary statebased peridynamics formulation for thermoplastic fracture. International Journal of Impact Engineering 2016;87: 83-94.

[39] Bobaru F, Ha YD. Adaptive refinement and multiscale modeling in 2D peridynamics. Journal for Multiscale Computational Engineering 2011: 635-59.

[40] Askari E, Bobaru F, Lehoucq R, Parks M, Silling S, Weckner O. Peridynamics for multiscale materials modeling. Journal of Physics: Conference Series: IOP Publishing; 2008. 012078.

[41] Oterkus E, Madenci E, Weckner O, Silling S, Bogert P, Tessler A. Combined finite element and peridynamic analyses for predicting failure in a stiffened composite curved panel with a central slot. Composite Structures 2012;94(3): 839-50. 
[42] Hu W, Ha YD, Bobaru F. Peridynamic model for dynamic fracture in unidirectional fiberreinforced composites. Computer Methods in Applied Mechanics Engineering 2012;217: 247-61. [43] Oterkus E, Madenci E. Peridynamic theory for damage initiation and growth in composite laminate. Key Engineering Materials: Trans Tech Publ; 2012. 355-58.

[44] Oterkus E. Peridynamic theory for modeling three-dimensional damage growth in metallic and composite structures. The University of Arizona: The University of Arizona; 2010.

[45] Silling SA, Askari A. Peridynamic model for fatigue cracking. SAND-18590 Albuquerque: Sandia National Laboratories 2014.

[46] Zhang G, Le Q, Loghin A, Subramaniyan A, Bobaru F. Validation of a peridynamic model for fatigue cracking. Engineering Fracture Mechanics 2016;162: 76-94.

[47] Jung J, Seok J. Mixed-mode fatigue crack growth analysis using peridynamic approach. International Journal of Fatigue 2017;103: 591-603.

[48] Liu W, Hong J-W. A coupling approach of discretized peridynamics with finite element method. Computer methods in applied mechanics engineering 2012;245: 163-75.

[49] Bie Y, Cui X, Li Z. A coupling approach of state-based peridynamics with node-based smoothed finite element method. Computer Methods in Applied Mechanics Engineering 2018;331: 675-700.

[50] Kilic B, Madenci E. Coupling of peridynamic theory and the finite element method. Journal of mechanics of materials structures 2010;5(5): 707-33.

[51] Yang Z, Oterkus E, Nguyen CT, Oterkus S. Implementation of peridynamic beam and plate formulations in finite element framework. Continuum Mechanics Thermodynamics 2019;31(1): $301-15$.

[52] Macek RW, Silling SA. Peridynamics via finite element analysis. Finite Elements in Analysis Design 2007;43(15): 1169-78.

[53] Diyaroglu C, Oterkus S, Oterkus E. A novel finite element technique for moisture diffusion modeling using ANSYS. 2018 IEEE 68th Electronic Components and Technology Conference (ECTC): IEEE; 2018. 227-35.

[54] Han S, Diyaroglu C, Oterkus S, Madenci E, Oterkus E, Hwang Y, Seol H. Peridynamic direct concentration approach by using ANSYS. 2016 IEEE 66th Electronic Components and Technology Conference (ECTC): IEEE; 2016. 544-49.

[55] Silling SA, Bobaru F. Peridynamic modeling of membranes and fibers. International Journal of Non-Linear Mechanics 2005;40(2-3): 395-409.

[56] O'Grady J, Foster J. Peridynamic plates and flat shells: A non-ordinary, state-based model. International Journal of Solids Structures 2014;51(25-26): 4572-79.

[57] Diyaroglu C, Oterkus E, Oterkus S, Madenci E. Peridynamics for bending of beams and plates with transverse shear deformation. International Journal of Solids Structures 2015;69: 15268.

[58] Nguyen CT, Oterkus S. Investigating the effect of crack propagation on strength of ship structures by using peridynamics. Manuscript submitted for publication.

[59] Yang Z, Vazic B, Diyaroglu C, Oterkus E, Oterkus S. A Kirchhoff plate formulation in a state-based peridynamic framework. Mathematics and Mechanics of Solids 2020;25(3): 727-38. [60] Yang Z, Oterkus E, Oterkus S. A state-based peridynamic formulation for functionally graded Kirchhoff plates. Mathematics and Mechanics of Solids 2020.

[61] Yang Z, Oterkus E, Oterkus S. Peridynamic mindlin plate formulation for functionally graded materials. Journal of Composites Science 2020;4(2): 76. 
[62] Silling SA, Zimmermann M, Abeyaratne R. Deformation of a peridynamic bar. Journal of Elasticity 2003;73(1-3): 173-90.

[63] O'Grady J, Foster J. Peridynamic beams: a non-ordinary, state-based model. International Journal of Solids Structures 2014;51(18): 3177-83.

[64] Diyaroglu C, Oterkus E, Oterkus S. An Euler-Bernoulli beam formulation in an ordinary state-based peridynamic framework. Mathematics Mechanics of Solids 2019;24(2): 361-76.

[65] Yang Z, Oterkus E, Oterkus S. A state-based peridynamic formulation for functionally graded Euler-Bernoulli beams. CMES-Computer Modeling in Engineering and Sciences 2020;124(2): 527-44.

[66] Nguyen CT, Oterkus S. Peridynamics formulation for beam structures to predict damage in offshore structures. Ocean Engineering 2019;173: 244-67.

[67] Kilic B, Madenci E. An adaptive dynamic relaxation method for quasi-static simulations using the peridynamic theory. Theoretical Applied Fracture Mechanics 2010;53(3): 194-204. [68] Timoshenko SP, Goodier JN. Theory of Elasticity. London: McGraw-Hill, New York.: McGraw Hill; 1970. 403-08.

[69] Boresi AP, Schmidt RJ, Sidebottom OM. Advanced mechanics of materials: Wiley New York et al.; 1985.

[70] Foster JT, Silling SA, Chen W. An energy based failure criterion for use with peridynamic states. International Journal for Multiscale Computational Engineering 2011;9(6).

[71] Nguyen CT, Oterkus S. Investigating the effect of brittle crack propagation on the strength of ship structures by using peridynamics. Ocean Engineering 2020;209: 107472.

[72] Silling S. EMU user's manual, Code Ver. 2.6 d. Sandia National Laboratories, Albuquerque 2004.

[73] Underwood P. Dynamic relaxation. Computational method for transient analysis 1986;1: 245-63.

[74] Pai P, Palazotto A. Large-deformation analysis of flexible beams. International Journal of Solids and Structures 1996;33(9): 1335-53.

[75] Bathe KJ, Bolourchi S. Large displacement analysis of three-dimensional beam structures. International journal for numerical methods in engineering 1979;14(7): 961-86.

[76] Heisser RH, Patil VP, Stoop N, Villermaux E, Dunkel J. Controlling fracture cascades through twisting and quenching. Proceedings of the National Academy of Sciences 2018;115(35): 8665-70.

[77] Guinea G, Rojo F, Elices M. Brittle failure of dry spaghetti. Engineering Failure Analysis 2004;11(5): 705-14.

[78] Diyaroglu C. Peridynamics and its applications in marine structures: University of Strathclyde; 2016. 